\title{
Vulnerability Analysis of the Wireless Infrastructure to \\ Intentional Electromagnetic Interference
}

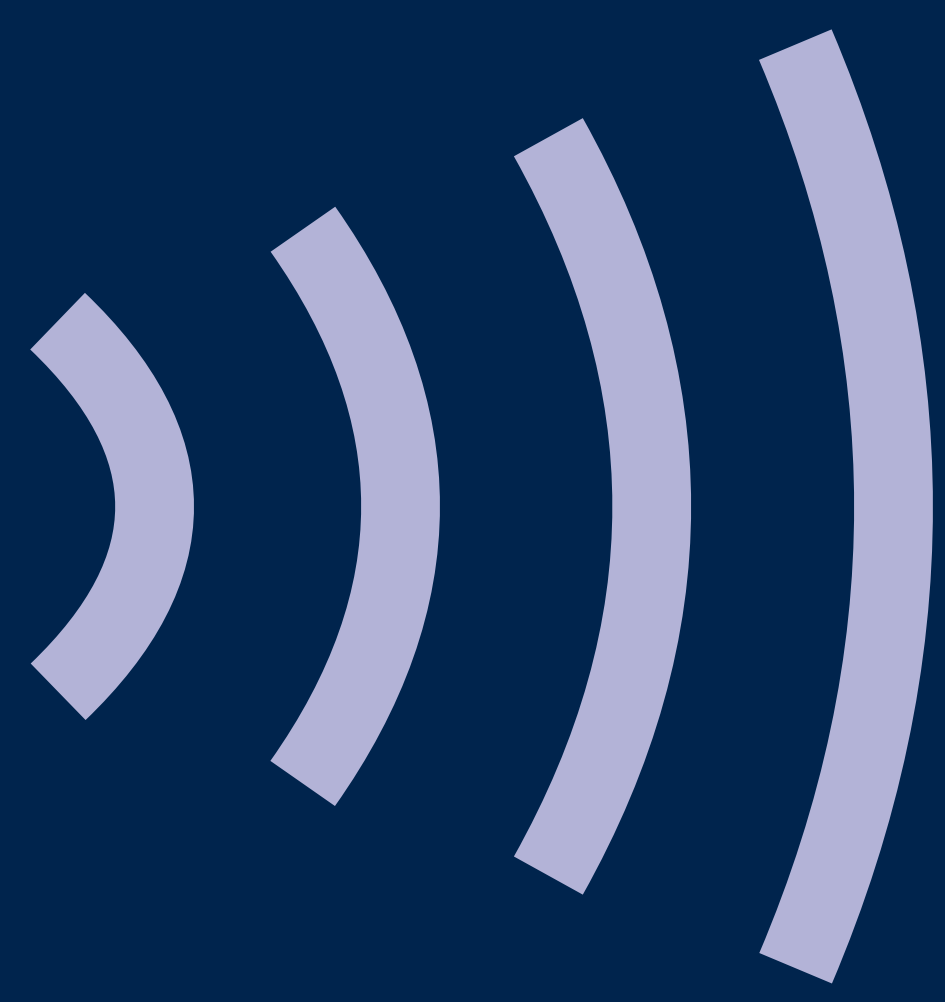

Stefan van de Beek 


\section{Vulnerability Analysis of the WIRELESS INFRASTRUCTURE TO InTENTIONAL ElECTROMAGNETIC INTERFERENCE}

Stefan van de Beek 
Samenstelling promotiecommissie:

Voorzitter en secretaris:

Prof.dr. P.M.G. Apers

Universiteit Twente

Promotor:

Prof.dr.ir. F.B.J. Leferink

Universiteit Twente Leden:

Dr.ir. M.J. Bentum

Universiteit Twente

Prof.dr.ir. B. Nauta

Universiteit Twente

Dr.ir. A.B.J. Kokkeler

Universiteit Twente

Prof.dr.-ing. H. Garbe

Leibniz Universität Hannover

Prof.dr. F. Silva

Universitat Politècnica de Catalunya

Dr. R. Serra

Technische Universiteit Eindhoven

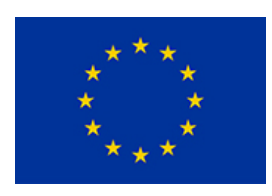

This research has received funding from the European Unions Seventh Framework Programme for research, technological development and demonstration under grant agreement No. FP7-SEC-2011-285257.

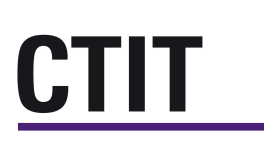

CTIT Ph.D. Thesis Series No. 16-413

Centre for Telematics and Information Technology P.O. Box 217, 7500 AE

Enschede, The Netherlands.

ISBN: $\quad 978-90-365-4252-4$

ISSN: $\quad$ 1381-3617 (CTIT Ph.D. Thesis Series No. 16-413)

DOI: $\quad 10.3990 / 1.9789036542524$ (https://doi.org/10.3990/1.9789036542524)

Copyright (C) 2016 by Stefan van de Beek, Enschede, The Netherlands.

All rights reserved.

Typeset with $\mathrm{LT}_{\mathrm{E} X}$.

This thesis was printed by Gildeprint Drukkerijen, The Netherlands. 


\section{Vulnerability AnAlysis of The Wireless InFRASTRUCTURE TO InTENTIONAL ElECTROMAGNETIC INTERFERENCE}

\section{PROEFSCHRIFT}

ter verkrijging van

de graad van doctor aan de Universiteit Twente, op gezag van de rector magnificus, prof. dr. H. Brinksma, volgens besluit van het College voor Promoties in het openbaar te verdedigen op donderdag 17 november 2016 om 16:45 uur

door

Gerrit Stefan van de Beek geboren op 16 maart 1988 te Voorthuizen 
Dit proefschrift is goedgekeurd door:

de promotor prof.dr.ir. F.B.J. Leferink 


\section{Samenvatting}

De hedendaagse maatschappij is sterk afhankelijk van een aantal kritieke infrastructuren (critical infrastructures CI) die een bijdrage leveren aan onze veiligheid en levenskwaliteit. Elektronische systemen regelen de veiligheidskritische functies van de meeste CIs en deze systemen zijn vatbaar voor elektromagnetische interferentie (EMI). Een gevaar voor infrastructuren is dat kwaadwillenden, zoals terroristen, het functioneren kunnen ontregelen door het gebruik van elektromagnetische stoorbronnen. Dit is gedefinieerd als bewuste elektromagnetische interferentie (intentional EMI IEMI). De Europese Commissie heeft als reactie hierop een onderzoeksoproep gedaan voor het beschermen van CIs tegen elektromagnetische (EM) aanvallen. Het project STRUCTURES - voorgesteld door een Europese consortium-heeft deze oproep geadresseerd en is gefinancierd. Het werk dat in dit proefschrift gepresenteerd wordt, is uitgevoerd binnen STRUCTURES.

Het doel van dit proefschrift is om de kwetsbaarheid van de bestaande draadloze communicatie infrastructuur tegen IEMI te onderzoeken. Draadloze communicatie wordt vandaag de dag over de hele wereld gebruikt en de afhankelijkheid van de maatschappij van draadloze netwerken groeit. Communicatie is essentieel voor het veilig en effectief functioneren van de hulpdiensten en hiermee dus voor de veiligheid van de burgers. Een gedetailleerd inzicht in de kwetsbaarheid van draadloze systemen zou moeten resulteren in de identificatie van de juiste beschermingsstrategieën en tegenmaatregelen om de robuustheid van de CI te verhogen. De ontwikkeling van nieuwe beschermingstechnieken is geen onderdeel van dit onderzoek.

De kwetsbaarheidsanalyse van de draadloze communicatie infrastructuur in dit proefschrift start met een dreigingsanalyse van een IEMI aanval. Voor een volledige analyse is het nodig om, naast de technische attributen, ook rekening te houden met de waarschijnlijkheid van een IEMI aanval. Er wordt geconcludeerd dat IEMI een serieuze dreiging is voor draadloze communicatie door de hoge kwetsbaarheid van de draadloze link en de ontvangers en door een hoge waarschijnlijkheid van een IEMI aanval. Vervolgens wordt de vatbaarheid van de draadloze communicatiesystemen geanalyseerd. Er worden drie verschillende interferentiemechanismen herkend-fysieke beschadiging van de ontvanger, verzadiging van de ontvanger, en jamming - die kun- 
nen resulteren in een denial-of-service (DoS) van het systeem. Generieke experimentele methoden die gebruikt kunnen worden om experimenteel de vatbaarheidsniveaus te testen worden gepresenteerd en een terrestrial trunked radio (TETRA) basisstation wordt onderzocht. De basisstations zijn niet uitgerust met RF limiters, waardoor ze kwetsbaar zijn voor fysieke beschadiging van de ontvanger. Het is geconcludeerd dat de interferentiemechanismen fundamenteel van elkaar verschillen en dat de beschermingsstrategieën afzonderlijk moeten worden geadresseerd. Hiervoor zou een robuust communicatiesysteem ontwikkeld moeten worden door experts van verscheidene disciplines zoals EMC experts, radio ingenieurs, antenne ingenieurs en chip ontwerpers.

Hierna wordt de kwetsbaarheid van TETRA voor intelligente jamming technieken onderzocht. Intelligente jammers zijn ontwikkeld om de doeltreffendheid van een aanval te verhogen gespecificeerd met criteria zoals energie efficiëntie, waarschijnlijkheid van opsporing, niveau van DoS, en weerstand tegen anti-jamming technieken op de fysieke laag. Na analyse van het TETRA protocol wordt er geconcludeerd dat het TETRA kwetsbaar is tegen een intelligente jammer. Het 'slotted Aloha' protocol kan verstoord worden door het 'access assignment channel' blok te corrumperen. Het TETRA protocol beschrijft dat het mobiele station voor onbepaalde tijd zal wachten met zenden, totdat het access assignment channel kan worden gedecodeerd. Vervolgens wordt de kwetsbaarheid van sleutelloze toegangssystemen (remote keyless-entry RKE) onderzocht. Een RKE systeem is een elektronisch slot dat de toegang tot voertuigen of gebouwen regelt door middel van een draadloze sleutel gedragen door de gebruiker. Ook al worden de systemen steeds beter beveiligd door middel van encryptie en coderingsalgoritmes, ze blijven kwetsbaar tegen hacking technieken die gebaseerd zijn op het jammen van de draadloze link van de sleutel naar de ontvanger, terwijl de aanvaller tegelijkertijd de mogelijkheid heeft om het signaal van de sleutel te ontvangen. Vooral RKE ontvangers met een slechte selectiviteit zijn kwetsbaar tegen deze hacking techniek. Uit dit onderzoek blijkt dat ontvangers met omhullende detectors ook erg kwetsbaar zijn door de hoge gevoeligheid tegen gepulste interferentie. Er wordt geconcludeerd dat een verbeterd RKE systeem gebruik zou moeten maken van een uiterst selectieve ontvanger met een synchrone ontvanger.

Een van de interferentiemechanismen - verzadiging van de ontvanger door een sterk stoorsignaal (blocker)—wordt vervolgens verder onderzocht. Een experimentele methode die gebruikt kan worden om de effecten van een blocker op de prestaties van de ontvanger te meten wordt gepresenteerd. Deze methode wordt vervolgens gebruikt om een commerciële lage-ruis-versterker (low noise amplifier LNA) te karakteriseren. De schadelijke effecten die plaatsvinden in de RF stage van de ontvanger worden vertaald naar de impact het heeft op de systeemprestaties wat betreft de bitfout waarschijnlijkheid. Recente ontwikkelingen op het gebied van geïntegreerde circuit technieken hebben geresulteerd in radio ontvanger architecturen die robuust zijn tegen blockers. 
Ten slotte wordt er een methode gepresenteerd die toegepast kan worden om de vereiste beschermingsniveaus voor kritieke apparatuur tegen IEMI in te schatten. Voorts wordt er een methode gepresenteerd om de kosten van het implementeren van een beschermingstechniek te analyseren. Deze generieke methodes worden vervolgens toegepast op de draadloze infrastructuur, maar ze kunnen worden toegepast op elke infrastructuur.

Algeheel kan er worden geconcludeerd dat in dit proefschrift een gedetailleerde risicoanalyse van IEMI tegen draadloze communicatie is gepresenteerd. Er zijn verscheidene redenen geïdentificeerd waarom IEMI als een serieuze dreiging tegen draadloze communicatie zou moeten worden beschouwd. Een uitgebreide kwetsbaarheidsanalyse is gepresenteerd en verschillende generieke experimentele methodes zijn getoond. Verscheidene beschermingstechnieken voor de verschillende interferentiemechanismen zijn geïdentificeerd en dit kan gebruikt worden om draadloze communicatie robuuster te maken tegen IEMI. 


\section{Summary}

Contemporary society is greatly dependent upon a set of critical infrastructures (CIs) providing security and quality of life. Electronic systems control the safety-critical functioning of most CIs, and these electronic systems are susceptible to electromagnetic interference (EMI). A threat to the infrastructures is that adversaries, such as terrorists, could disrupt the functioning by using electromagnetic (EM) sources. This is defined as intentional electromagnetic interference (IEMI). The European Commission released a research call to protect the CIs against EM attacks, and the project STRUCTURES - lead by an European consortium-addressed this call and got funded. The work presented in this thesis was conducted within STRUCTURES.

The research goal of this thesis is to study the vulnerability of the wireless communication infrastructure to IEMI. Wireless communication is today being used all over the world and the dependence of society upon wireless networks is growing. Communication is essential for the safe and effective functioning of the emergency services and herewith for the safety of the civilians. A detailed insight into the vulnerability of wireless systems should result into the identification of proper protection strategies and countermeasures to increase the robustness of the CI. The development of new innovative protection techniques is not part of this work.

The vulnerability analysis of the wireless communication infrastructure in this thesis starts with a threat analysis of an IEMI attack. It is necessary to not only look at technical attributes such as susceptibility levels, but also take the likelihood of an IEMI event into account. It is concluded that IEMI is a serious threat for wireless communication due to the high vulnerability of the wireless link and the wireless receivers, and the high likelihood of an IEMI attack. The susceptibility of wireless communication systems is analyzed next. Three different interference mechanisms are recognized - physical damage of the receiver, saturation of the receiver, and jamming - that could result into a denial-of-service (DoS) of the system. Generic experimental methods are presented that can be used to experimentally test the susceptibility levels of wireless receivers, and a terrestrial trunked radio (TETRA) base station is investigated. The base stations are not equipped with RF limiters, rendering them vulnerable to physical damage of the receiver. It is concluded that the inter- 
ference mechanisms are fundamentally different and protection strategies need to be addressed separately. Therefore a robust communication system should be designed by experts from various disciplines such as EMC experts, radio engineers, antenna engineers, and microwave engineers.

Next, the vulnerability of TETRA against intelligent jamming is investigated. Intelligent jammers have been developed to increase jamming efficiency by criteria such as energy efficiency, probability of detection, level of DoS, and resistance to physical layer anti-jamming techniques. From analysis of the TETRA protocol it is concluded that it can be disrupted by an intelligent jammer. The slotted ALOHA protocol can be interfered by corrupting each access assignment channel block, since the TETRA protocol states that the mobile station will wait indefinitely before transmitting until the access assignment channel can be decoded. The vulnerability of remote keylessentry (RKE) systems to jamming attacks is subsequently investigated. An RKE system is an electronic lock that controls access to vehicles or buildings by use of a wireless key fob carried by the user. Even though the systems are increasingly secured by use of encryption and code algorithms, they are still susceptible to hacking attacks that rely on jamming the wireless link from the key fob to the receiver, while the attacker is able to receive the signal from the key fob. Especially receivers with a poor selectivity are vulnerable to this hacking technique. This research shows that receivers equipped with envelope detectors are also vulnerable due to the high vulnerability against pulsed interference. It is concluded that an improved RKE system would use a highly selective receiver with a synchronous detector.

One of the interference mechanisms, saturation of the receiver due to a blocker, is then investigated. An experimental method is presented that can be used to measure the effects of a blocker on the performance of a receivers front end. This method was used to characterize a commercial-off-the-shelf (COTS) LNA. The detrimental impact at the RF stages is translated to the effects it has on the system performance in terms of bit-error-probability (BEP). Recent developments in the field of solid-state circuits resulted in more robust receiver architectures against blockers.

Finally, a methodology is presented for estimating the required protection levels of critical equipment against IEMI. Furthermore, a method to analyze the cost of implementing a specific protection technique is presented. These generic methods are applied to the wireless infrastructure, but they can be applied to any infrastructure.

Overall, it can be concluded that a detailed risk analysis of IEMI against wireless communication is presented in this thesis and various reasons are identified why IEMI should be considered as a serious threat for wireless communication. A comprehensive vulnerability analysis is presented and along this analysis generic experimental methods are shown. For the three different interference mechanisms, various protection techniques and strategies are identified, which can be used to improve the robustness of wireless communication against IEMI. 


\section{Contents}

Samenvatting $\quad$ i

Summary $\quad$ v

1 Introduction $\quad 1$

1.1 Motivation .......................... 1

1.2 Research project - STRUCTURES . . . . . . . . . . . . . 4

1.3 Wireless communication . . . . . . . . . . . . . 7

1.4 Research goals . . . . . . . . . . . . . . . . 9 9

1.5 Outline of the thesis . . . . . . . . . . . . . 9

2 Threat analysis $\quad 11$

2.1 Description of an IEMI scenario . . . . . . . . . . . . . . . . . . 11

2.2 Analysis of the IEMI sources . . . . . . . . . . . . . . . . . . . 12

2.2.1 Classification based on technical attributes . . . . . . . . . 13

2.2 .2 Risk potential of IEMI source . . . . . . . . . . . . . . 16

2.2 .3 Literature survey . . . . . . . . . . . . . . . . . 18

2.3 Coupling of IEMI . . . . . . . . . . . . . . . . . . . . . . . . . . . . . . 18

2.3.1 Front door coupling . . . . . . . . . . . . . . 19

2.3.2 Back door coupling . . . . . . . . . . . . . . . 20

2.4 Critical infrastructures . . . . . . . . . . . . . . . 20

2.4 .1 Accessibility . . . . . . . . . . . . . . 21

2.4 .2 Consequence ........................ 22

2.4 .3 Susceptibility . . . . . . . . . . . . . . 22

2.5 Wireless communication infrastructure . . . . . . . . . . . . 24

2.5.1 Overview of a typical wireless instrastructure . . . . . . . . 24

2.5.2 Analysis of the IEMI threat for wireless communication . . . . 24

2.6 Summary and conclusions . . . . . . . . . . . . . . . 26 
3 Interference mechanisms $\quad 27$

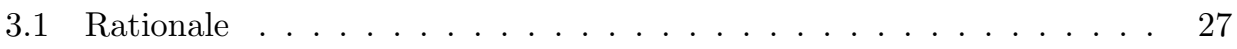

3.2 Overview of typical wireless receiver . . . . . . . . . . . . . . 28

3.3 TETRA overview . . . . . . . . . . . . . . . . . . . 29

3.3.1 Air interface . . . . . . . . . . . . . . . . 29

3.3.2 Typical base station structure . . . . . . . . . . . . . . 30

3.4 Analysis on front door coupled IEMI . . . . . . . . . . . . . . . . . . 31

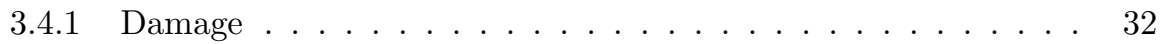

3.4.2 Saturation . . . . . . . . . . . . . . . 34

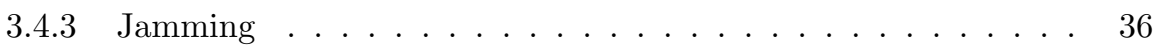

3.5 Experimental testing method . . . . . . . . . . . . . . 37

3.5.1 Gain compression . . . . . . . . . . . . . . 38

3.5.2 BER .......................... 40

3.6 Experimental results . . . . . . . . . . . . . . . . . . . . . . . . 41

3.6.1 Gain compression . . . . . . . . . . . . . . 41

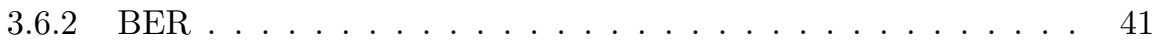

3.7 Discussion . . . . . . . . . . . . . . . . . . . . . . . . . . . 44

3.8 Summary and conclusions . . . . . . . . . . . . . . . 46

4 Intelligent jamming $\quad 47$

4.1 Background of intelligent jamming attacks . . . . . . . . . . . . . . 47

4.2 Vulnerabilities of TETRA protocol . . . . . . . . . . . . . . 49

4.2.1 Interfering with the voice data . . . . . . . . . . . 49

4.2.2 Distributed Denial of Service (DDoS) attacks . . . . . . . . . . 50

4.2.3 Interfering with the TDMA synchronisation . . . . . . . . . 50

4.2.4 Interfering with the Access Assignment Channel . . . . . . . . 51

4.3 Symbol errors on the physical layer due to interference signals . . . . . 52

4.4 Intelligent TETRA jammer . . . . . . . . . . . . . . . . 54

4.5 Experimental results . . . . . . . . . . . . . . . . . 55

4.6 Summary and conclusions . . . . . . . . . . . . . . . 58

$5 \quad$ Jamming attacks against remote keyless-entry systems $\quad 61$

5.1 Background on RKE systems and IEMI . . . . . . . . . . . . . . . 61

5.2 Analysis of purchased low-cost RKE systems . . . . . . . . . . . 63

5.2.1 Super regenerative receivers . . . . . . . . . . . . . . 63

5.2 .2 Selectivity of purchased systems . . . . . . . . . . 64

5.2 .3 Discussion . . . . . . . . . . . . . . 66

5.3 Analysis of pulsed interference . . . . . . . . . . . . 66

5.3.1 Superheterodyne receivers in RKE . . . . . . . . . . . 66

5.3.2 Background on pulsed interference . . . . . . . . . . 66 
5.3.3 Simulation model of a general envelope detector . . . . . . . . 67

5.4 Experimental study of an RKE receiver . . . . . . . . . . . . . 70

5.4.1 Experimental method . . . . . . . . . . . . . . . 70

5.4 .2 Experimental results . . . . . . . . . . . . . . . . 72

5.5 Improved receiver type . . . . . . . . . . . . . . . 76

5.5.1 Vulnerability of receiver against pulsed interference . . . . . . 76

5.5.2 Synchronous detector . . . . . . . . . . . . . . . . 77

5.5.3 Simulations of improved performance. . . . . . . . . . . 77

5.6 Summary and conclusions . . . . . . . . . . . . . . . . 79

6 Blocking and desensitization $\quad 81$

6.1 Rationale . . . . . . . . . . . . . . . . . . . . 81

6.2 Blocking mechanisms . . . . . . . . . . . . . . . . 82

6.2.1 Non-linear effects . . . . . . . . . . . . . . . . 82

6.2.2 Desensitization .................... 84

6.3 LNA characterization . . . . . . . . . . . . . . . 86

6.3.1 Experimental set-up . . . . . . . . . . . . . 86

6.3.2 Gain compression ................. 86

6.3.3 Distorted spectrum . . . . . . . . . . . . . 88

6.3.4 Blocker noise figure . . . . . . . . . . . . . . . . . . 89

6.4 System performance . . . . . . . . . . . . . . . 91

6.4.1 Modulation formats and bit error probability . . . . . . . . . 91

6.4.2 BEP curves . . . . . . . . . . . . . . . . . 91

6.5 Discussion on possible improvements . . . . . . . . . . . . . . . . 93

6.6 Summary and conclusions . . . . . . . . . . . . . . . . 94

7 Protection strategies against IEMI $\quad 97$

7.1 Background on protection strategies . . . . . . . . . . . . . . 97

7.2 Procedure for estimating the required protection levels . . . . . . . . . 99

7.3 Required protection levels for a typical base station . . . . . . . . . . . 100

7.3.1 Topological decomposition of the IEMI scenario . . . . . . . . . 100

7.3 .2 IEMI source description . . . . . . . . . . . . . . . . 100

7.3.3 Obtaining the required protection level . . . . . . . . . . . 101

7.4 Identification of protection techniques . . . . . . . . . . . . . . . 102

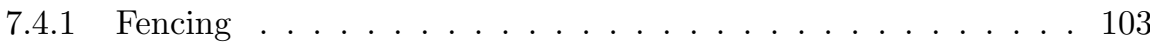

7.4 .2 RF limiter . . . . . . . . . . . . . . . . . 103

7.5 Evaluation of the protection technique . . . . . . . . . . . . . 104

7.5.1 Monetary costs . . . . . . . . . . . . . . . . . . . 104

7.5.2 Loss in performance . . . . . . . . . . . . . . . 106

7.6 Summary and conclusions . . . . . . . . . . . . . . 107 
8 Conclusions $\quad 109$

8.1 Summary and conclusions . . . . . . . . . . . . . . . . 109

8.2 Recommendations ..................... 111

$\begin{array}{ll}\text { References } & 113\end{array}$

$\begin{array}{ll}\text { List of Publications } & 127\end{array}$

$\begin{array}{ll}\text { List of Abbreviations } & 131\end{array}$

Dankwoord 135 


\section{Chapter 1}

\section{Introduction}

\subsection{Motivation}

Contemporary society is greatly dependent upon a set of critical infrastructures (CIs) providing security and quality of life. In [1], a definition of a CI is given as:

"an asset, system or part thereof [...] which is essential for the maintenance of vital societal functions, health, safety, security, economic or social well-being of people, and the disruption or destruction of which would have a significant impact [...] as a result of the failure to maintain those functions"

Examples of such infrastructures are [2]: telecommunication, electrical power systems, gas and oil storage and transportation, bank and finance, transportation, water supply systems, and emergency services. For obvious reasons, it is vital to protect these civilian CIs against external attacks by adversaries such as terrorists. The protection is highly complicated because CIs are generally largely distributed, complex, and interdependent. The interdependencies amongst CIs increases the risk of failure propagation to multiple infrastructures [3], which increases their vulnerability to attacks.

A steep increase in the use of electronics systems in civilian infrastructures has been seen over the last decades. Electric or electronic systems control the safetycritical functionality of a variety of CIs. For example, supervisory control and data acquisition (SCADA) is used for controlling and monitoring CIs and depends heavily on electronics. It is well known that the functioning of electronics can be disrupted or damaged by electromagnetic interference (EMI). This means that CIs are vulnerable against EMI, and an easily recognized threat is that adversaries could disrupt CIs using electromagnetic (EM) sources. This is defined as intentional electromagnetic 
interference (IEMI) and is described in [4] as:

"intentional malicious generation of EM energy introducing noise or signals into electric or electronic systems, thus disrupting, confusing, or damaging these systems for terrorist or criminal purposes"

IEMI is considered to be a serious risk for CIs and the reasons are twofold. Firstly, the previously mentioned increasing use of, and dependability on, electronics in CIs. Electronics are in general becoming more susceptible to EMI due to higher package densities and increasing use of the electromagnetic (EM) spectrum [5]. Secondly, we can observe a proliferation of powerful EM generators that can be adapted to IEMI sources [6]. Examples of widely available EM generators can be found in systems such as microwave ovens or civil radar systems.

To understand the risk of an electromagnetic attack against a CI, it is important to understand the physical effect that EMI can have on electric or electronic systems. The effect of EMI on systems has been thoroughly studied in the electromagnetic compatibility (EMC) world and are described in well-known books $[7,8]$. There are various electromagnetic environments (EME), either natural or man-made, that can disrupt or damage electronics. Examples of EMI and their effects on systems that are well studied are lightning strikes and the high-altitude electromagnetic pulse (HEMP). These studies brought forth tested technical knowledge and excellent standards describing the phenomena and protection strategies [9-16].

Nowadays commercial equipment are tested against product or generic EMC standards, but this does not mean it is robust against IEMI. This only means that it passed the standard EMC test, which does not include EM stresses that can be expected during an IEMI attack. Typical examples of well-known classical EMI originates for instance from major mobile communication technologies such as Global System for Mobile Communications (GSM), Universal Mobile Telecommunications System (UMTS), Long-Term Evolution (LTE) and WiFi. Typically, the EMI due to wireless systems from these technologies have expected field strengths below $1 \mathrm{~V} / \mathrm{m}$. Protection against IEMI is different from classical EMC, lightning and HEMP, and therefore requires additional research.

Technological advances resulted in IEMI sources capable of generating high-power electromagnetic (HPEM) fields with greater capability to disrupt systems [17]. The IEMI sources can generate both conducted and radiated interference, but in this thesis the focus is only on radiated interference. HPEM sources are now capable of generating output powers in the GW range [18]. In [19], a list is presented with documented system failures due to HPEM. The effects that HPEM can have on a system are hazardous, since it is able to induce conducted and radiated interference well above traditional interference levels. HPEM is typically defined as electromagnetic environments which produce radiated EM fields exceeding $100 \mathrm{~V} / \mathrm{m}$ or conducted voltages 


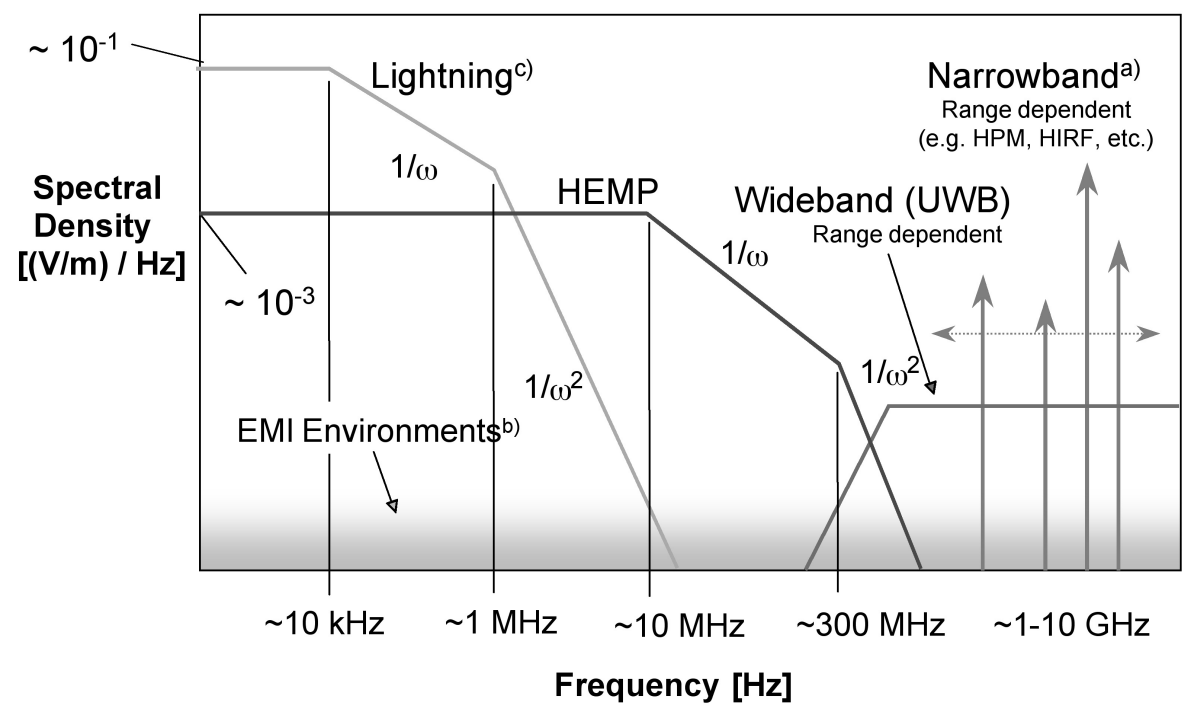

a) narrow band extending from $\sim 0,2$ to $\sim 5 \mathrm{GHz}$

b) not necessarily HPEM

c) significant spectral components up to $\sim 10 \mathrm{MHz}$ depending on range and application

Figure 1.1: HPEM environment and other electromagnetic environments. Adopted from $[5]$.

exceeding $1 \mathrm{kV}[5]$.

HPEM environments can be divided into two major categories: narrowband and wideband. Narrowband interference has the most energy concentrated at one specific frequency and is often referred to as high power microwave (HPM). Wideband interference on the other hand, spread its energy over a large frequency band and is often referred to as ultra-wideband (UWB). HPEM, both narrowband and wideband, is graphically compared to other well-known EME in Figure 1.1. It shows that it extends to higher frequencies than lightning and HEMP, and has more power than the classical EMI.

The differences between HEMP and HPEM are described in [20]. The main differences can be found in their spatial coverage and frequency range. Whereas the EM fields generated by HEMP can illuminate a whole continent, the radiation of the antenna of an HPEM source is only concentrated on a limited target region. Besides that, the HEMP signal only extends to a maximum of $300 \mathrm{MHz}$, while HPEM can extend to much higher frequencies (in the 1-10 $\mathrm{GHz}$ range).

It is now well established that EMI in the frequency range of $200 \mathrm{MHz}$ up to 5 $\mathrm{GHz}$ can be very effective in generating upsets or damage to electronic systems for 
the following reasons [5]:

- Many antennas operate in this frequency range (from $200 \mathrm{MHz}$ and up), providing a point of entry for interfering signals;

- Physical dimensions of circuit boxes are resonant in the frequency range of 1 to $3 \mathrm{GHz}$. Also, typical apertures, slots, holes, and hatch openings have their resonance in this frequency range;

- The interior coupling paths are roughly a quarter to a full wavelength in the frequency range of $1 \mathrm{GHz}$ to $3 \mathrm{GHz}$.

In [21], it is shown that the electromagnetic response of most systems is maximized around $1 \mathrm{GHz}$.

The effects of IEMI at a CI can be diverse and depends on the victim susceptibility. The effect of an electromagnetic attack can be classified into four different classes:

- Permanent damage;

- Upset;

- Interference;

- Deception.

Classification schemes are described in [22]. The most severe effect is damage, where the system needs repair before it can function again. In the case of an upset, the system is temporarily disrupted, but not damaged. Interference degrades the functioning of the system only during the attack, i.e., once the attack stops the system functions as specified again. Another effect that can be realized with an electromagnetic attack is deception. Systems such as the global positioning system (GPS) can be spoofed by transmitting a false signal [23].

In the last decade, several studies have investigated the impact of IEMI on individual electronic systems. In [24-26] the effect of HPEM on information technology (IT) equipment has been tested. The propagation of HPEM pulses on power supply networks is investigated in [27-29]. Additional work on the susceptibility of various equipment and systems against IEMI can be found in [30-35]. The research in this field resulted in the production (still ongoing) of several standards providing recommendations and protection guidelines for protection against HPEM by the subcommittee SC77C of International Electrotechnical Commission (IEC) [5, 19, 36-39].

\section{$1.2 \quad$ Research project - STRUCTURES}

The trends described in the previous section resulted in a research call from the European Commission to ensure the security of the citizens from threats posed by 
IEMI. The call is within the Security theme of the Seventh Framework Programme for European Research (FP7), and the topic is SEC-2011.2.2-2: Protection of Critical Infrastructure (structures, platforms and networks) against Electromagnetic (High Power Microwave (HPM)) Attacks. Three different European consortia addressed this call and got funded:

- STRUCTURES: Strategies for the Improvement of Critical Infrastructures Resilience to Electromagnetic Attacks [40];

- HIPOW: Protection of Critical Infrastructures against High Power Microwave Threats [41];

- SECRET: Security of Railways against Electromagnetic Attacks [42].

More information on European IEMI studies can be found in [43]. The research presented in this thesis was conducted as a part of the STRUCTURES project. A general overview of this project is presented in [44] and [45].

The starting date of the STRUCTURES project was 1 July 2012, and it ended at 30 October 2015. The consortium consisted of 13 partners, including several universities, companies and research centres. STRUCTURES aims at: analysing possible effects of EM attacks on critical infrastructures, assessing the impact for our defence and economic security, identifying innovative awareness and protection strategies, and at providing a picture for the policy makers on the possible consequences of an EM attack. The investigation was divided into three phases as can be seen in Figure 1.2. A managing and a dissemination work package (WP) ran along the whole duration of the project.

In the first phase, the focus was on the assessment of the scenario concerning IEMI attacks. For the physical scenario assessment, an extensive literature review was conducted to identify and classify possible IEMI threats and analyse the target systems. Analysis of the target systems and their criticality is of key importance to effectively use the limited resources on research. Ambiguity about criticality could result into inefficient research and focus on too many systems, or otherwise, focus on too little systems and miss a vulnerability [2]. Six critical infrastructures were analysed in the STRUCTURES project:

1. Power plant;

2. Communication exchange;

3. Transport based on train;

4. Bank/financial office;

5. Airport; 
WP1

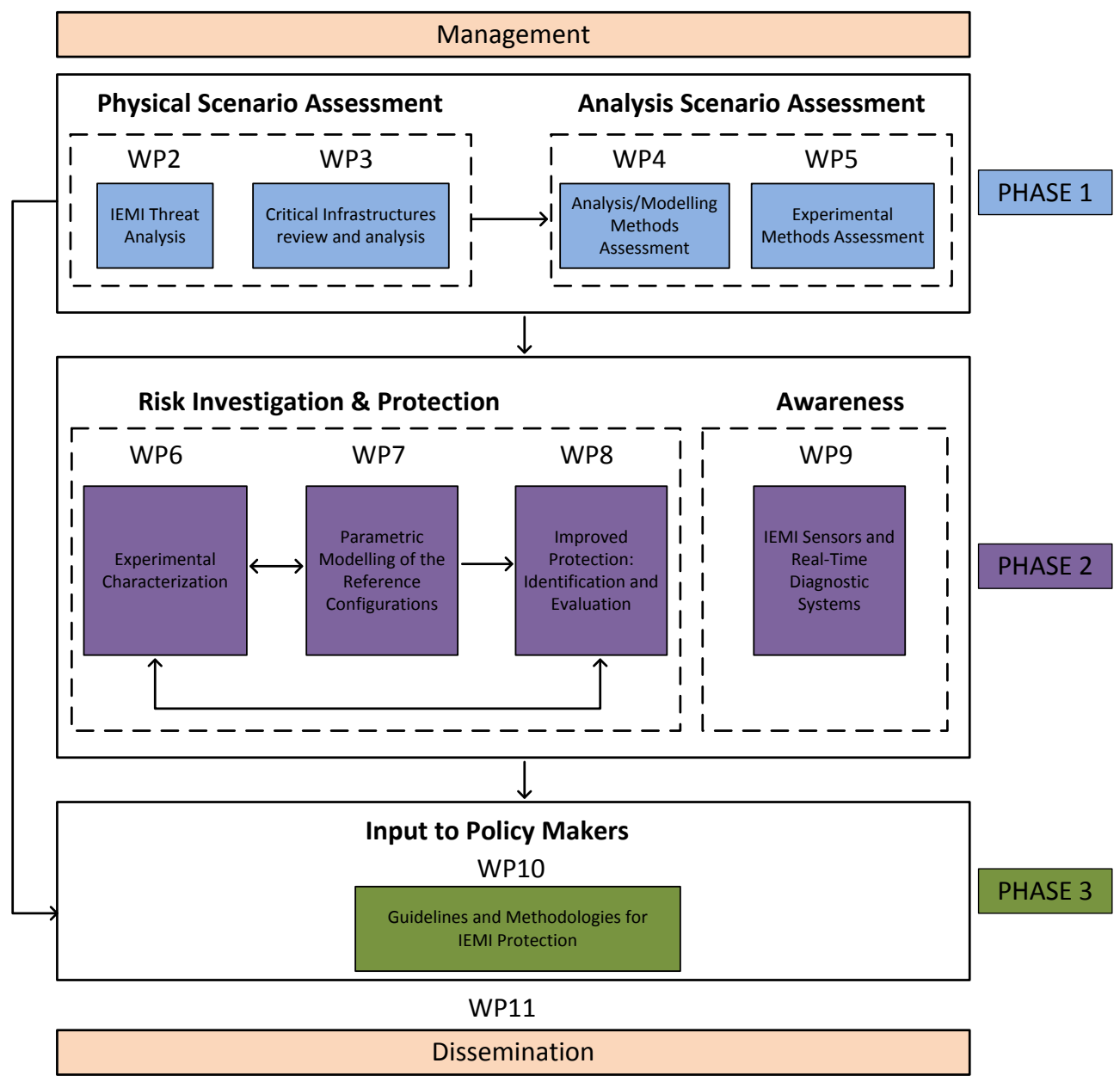

Figure 1.2: Overview of the STRUCTURES project.

\section{Computer network.}

In the analysis scenario assessment, the available experimental and simulation methods to model the relevant scenarios were investigated. In this thesis, the main focus will be on the wireless communication infrastructure. A part of the physical scenario assessment will be presented in Chapter 2 .

The second phase concerns the risk investigation, both experimental and numerical, and the proposal of possible protection strategies. Another part was dedicated to the awareness of victim systems regarding IEMI attacks. IEMI sensors were developed for real-time detection and identification of attacks. In this thesis, Chapter 3 to Chapter 6 present a detailed risk investigation for various wireless communication systems 
and identifies possible protection techniques. Chapter 7 presents a general protection strategy regarding IEMI threats which can be applied to any infrastructure.

In the third phase, the dissemination of the work and results of this project were addressed. All outcomes were combined and processed to define a series of guidelines for policy makers.

\subsection{Wireless communication}

This thesis focusses mainly on the threat of IEMI against wireless communication and the work presented here is part of the STRUCTURES project. Most critical infrastructures rely on wireless communication in one way or another. For instance, in the transport sector, systems such as air planes and vessels rely for there positioning and approach on GPS. Wireless communication systems are considered to be of key interest when investigating the effects of IEMI on infrastructures. As stated in the 'Technology Trends Survey' report from NATO published in 2015 [46], the most serious effects of HPM weapons will be on sensors working in the radio frequency (RF) region. Every device capable of wireless communication is equipped with a sensor working in the RF region, that is, an antenna.

Wireless communication of today is being used all over the world and the dependence of society upon wireless networks is growing. All sorts of critical services are provided by these networks, such as banking transactions, managing transportation, exchanging position information, and communication among safety organizations. Concepts such as smart cities, using information and communication technologies, and Internet of Things (IoT) are being developed to enhance quality and performance of urban services. These developments are highly dependent upon wireless communication. Machine-to-machine communication, for example car-to-car, is of particular interest and is expected to be commonplace in the near future. In a forecast released by Ericsson in 2015, it is estimated that the number of connected devices will reach 28 billion in 2021 [47]. These trends will be supported by the development of the fifth generation of mobile telecommunications $(5 \mathrm{G})$ and by updating existing mobile standards. Current major technologies are the 4 generations $(1 \mathrm{G} / 2 \mathrm{G} / 3 \mathrm{G} / 4 \mathrm{G})$ of mobile technologies, WiFi based on the IEEE $802.11 \mathrm{~b} / \mathrm{g} / \mathrm{n}$ protocol, ZigBee based on the IEEE 802.15.4 protocol, Bluetooth, and Terrestrial Trunked Radio (TETRA). The technology that is deployed by a system depends on the typical application and factors such as power demands, data requirements, range, and security. The arrival of new communication standards are promising increased benefits in terms of performances, possible services and amount of data that can be exchanged.

Wireless communication is fundamentally based on microwave technology and the propagation of EM waves through free space within the microwave frequency range. 
Microwave systems offers huge advantages such as providing the possibility for lineof-sight (LoS) communication and the support of wide bandwidth communication for high data rates. The majority of applications of microwave technology is communication systems, but there are more such as: radar systems, navigation systems, video broadcast, radio astronomy and sensors. Communication systems greatly benefited from the development in microwave technologies that was originally performed for radar systems [48]. The receivers employed in all these applications are based on similar microwave techniques. Different frequency bands are allocated to different systems to prevent coexistence problems and provide electromagnetic compatibility. Nowadays, communication applications are generally operating from $100 \mathrm{MHz}$ up to $6 \mathrm{GHz}$, but with the new developments this is expected to extend up to $100 \mathrm{GHz}$ supported by developments in the fields of microwave technology [47].

The biggest advantages of wireless communication is the flexibility it provides to the end user. Additionally, it is often easier to implement and better affordable than wired communication. However, a disadvantage which is inherently related to wireless systems is the vulnerability against EMI, both intentional and unintentional. The systems are susceptible for denial-of-service (DoS) attacks due to an easy point of entry for IEMI, and the open access nature of the wireless medium. The coupling of the IEMI via an antenna is defined as front door coupling [31], and it is difficult to protect a system's electronics against this type of IEMI coupling. The antenna is the point of entry of the IEMI, but also of the desired signal, and therefore it is designed to capture as much EM energy as possible within a certain frequency band. This easy point of entry facilitates an HPM attack that can possibly damage the electronics of the receiver. The open access nature of the medium makes it easy for an adversary to jam the communication signal. Many RF jammers are available online that are designed to emit noise at specified frequency bands employed by certain communication systems [49].

Reliance and dependence on systems employing wireless links can be a possible weakness in today and tomorrow's society. Terrorists or other adversaries might endeavour to disrupt or damage the civilian communication infrastructures. Communication is essential for the safe and effective functioning of the emergency services and herewith for the safety of the civilians. As an example, in February 2009, a plane of the Turkish Airlines crashed near Amsterdam and the professional mobile radio (PMR) system of the emergency services failed [50]. First responders were severely limited in the communication with the emergency control room and were forced to switch to private mobile phones. Consequences of disrupting mission-critical communications can be severe both economically and physically. Critical communication systems, such as TETRA which is specifically designed for PMR, have high demands on security and measures, such as encryption and other code algorithms, are implemented. These security measures are mainly addressed at the higher open systems 
interconnection (OSI) layers. However, the act of IEMI disrupts the system at the fundamental physical layer, which renders many security measures implemented at higher OSI layers to be useless. Military communication systems are developed with a major focus on reliability and are hardened against this threat. However, civilian systems are designed to only meet the modest immunity levels as prescribed by normative standards and are mainly developed from a functional and cost-effective point of view. For this reason, protection is not a major issue and therefore many civilian systems remain vulnerable against IEMI.

\subsection{Research goals}

In order to increase the robustness and resilience of society against external threats such as IEMI, it is necessary to have a solid understanding of the risks which are involved. The research goal of this thesis is to study the vulnerability of the civilian communication infrastructure against IEMI. A realistic threat analysis on an IEMI attack should be able to provide policy makers with a picture for the risk of such an event. Possible effects that EM attacks can have on wireless communication systems will be thoroughly analyzed in order to assess the susceptibility. A detailed insight into the vulnerability of wireless systems should result into the identification of proper protection strategies and countermeasures to increase the robustness of the CI. However, the development of new innovative protection techniques is not part of this work.

The following tasks should be fulfilled to achieve the stated goals of this research

- Provide a risk analysis of the threat IEMI poses to the wireless communication infrastructure;

- Identify the most relevant interference mechanisms that can disrupt wireless communication;

- Develop generic evaluation methods that can be used to experimentally test the susceptibility levels;

- Develop a generic methodology to estimate the required protection levels;

- Identify protection strategies to increase the robustness of the wireless infrastructure.

\subsection{Outline of the thesis}

This thesis is structured in line with the research goals stated in the previous section. 
An overview and a risk analysis of an IEMI attack against a CI is presented in Chapter 2. Of particular interest is the threat IEMI poses to a wireless infrastructure. Next, in Chapter 3, the susceptibility levels of wireless communication are thoroughly investigated, and the relevant interference mechanisms are identified and briefly discussed. This chapter also presents generic evaluation methods to experimentally test the susceptibility levels, and the method is applied to a typical TETRA base station.

The identified interference mechanisms are further investigated in the next three chapters. This investigation provides a detailed insight into the vulnerability of wireless systems and proper protection strategies are identified. Chapter 4 presents the investigation of the vulnerability of TETRA against intelligent jamming techniques. Chapter 5 discusses the weaknesses of remote-keyless-entry (RKE) systems against jamming attacks, and improvements are suggested. In Chapter 6 , the effect of a highpower interferer - saturating the receiver - on the performance of wireless systems is discussed.

Chapter 7 is dedicated to protection strategies for critical infrastructures. First, a short summary of protection techniques for wireless communication systems, as already discussed throughout this thesis, is presented. Next, a methodology is presented for both the estimation of the required protection levels of critical equipment, and for the evaluation of the applicable protection techniques.

Finally, in Chapter 8, an comprehensive conclusion and summary of this work is presented. The thesis finishes with directions for further research. 


\section{Chapter 2}

\section{Threat analysis}

An overview of the threat analysis of an IEMI attack is presented in this chapter. The threat analysis is based on an extensive literature study. Parts of this study are published in the IEEE Electromagnetic Compatibility Magazine [44] and presented at the IEEE International Symposium on Electromagnetic Compatibility in 2014 and $2015[45,51]$.

\subsection{Description of an IEMI scenario}

A typical IEMI attack as envisioned by the European Commission and standardization committees is presented in Figure 2.1. An adversary or terrorist could transport a HPEM source into close vicinity of civilian infrastructures and disrupt the electronics systems. The robustness of an infrastructure against IEMI cannot be evaluated based on standard EMC tests, and a dedicated threat analysis is necessary. Genender et al. presented a method to systematically analyze the risk of a facility exposed to IEMI in [52]. The main objective of the analysis in [52] is to determine, both qualitatively and quantitatively, the risk of a failure of a system during an IEMI attack. The overall structure of the threat analysis is divided into three main elements:

1. the IEMI sources,

2. the coupling of the EM energy to the CI,

3. the vulnerability level of the CI.

The first two steps in the threat analysis give an estimate of the electromagnetic threat level at the victim. Comparing this threat level with the vulnerability of a CI gives an estimation of the robustness of an infrastructure against IEMI [19]. 


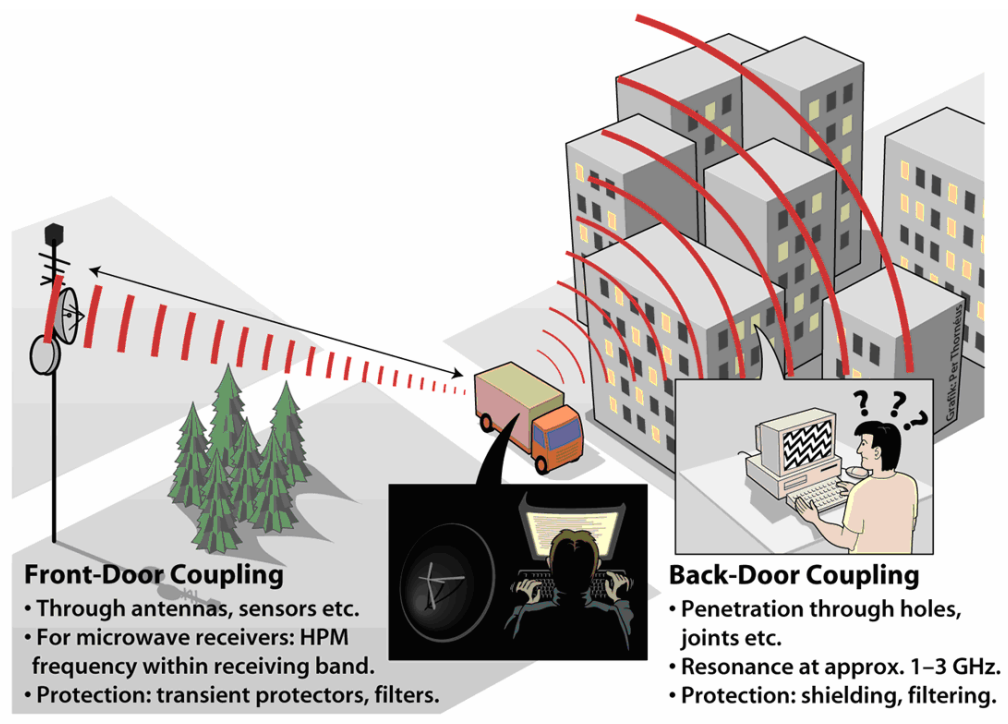

Figure 2.1: Typical envisioned scenario of an IEMI attack illustrating both front and back door coupling. Adopted from [31].

The analysis of the IEMI sources is not straightforward. The sources need to be classified according to both technical and non-technical parameters to examine the risk potential. A detailed discussion on IEMI sources is given in Section 2.2. The coupling of the EM energy to a highly distributed infrastructure is complex and there are many coupling paths that should be taken into account. The coupling of IEMI to a victim is discussed in Section 2.3. The vulnerability of the CI cannot simply be expressed as a defined EM level that causes an upset. Many additional attributes play a role and this will be further discussed in Section 2.4. Finally, in Section 2.5, an overview is given of a typical wireless infrastructure and a qualitative analysis of the risk IEMI poses to wireless communication is presented.

\subsection{Analysis of the IEMI sources}

Classification of sources capable of generating HPEM environments is an important step in the overall threat analysis of an IEMI attack [53]. The sources creating HPEM environments can be classified by many attributes, both technical and nontechnical. Technical attributes describe the physical characteristics and non-technical attributes focus more on the risk potential and addresses the likelihood of occurrence of an attack. The source attributes, both technical and non-technical, will be further discussed in the next two sections. 
Table 2.1: HPEM classification based on bandwidth.

\begin{tabular}{|c|c|c|}
\hline Band type & $\begin{array}{c}\text { Percent bandwidth } \\
p b w=200\left(\frac{b r-1}{b r+1}\right)(\%)\end{array}$ & Band ratio $\boldsymbol{b r}$ \\
\hline $\begin{array}{c}\text { narrow or } \\
\text { hypoband }\end{array}$ & $<1 \%$ & $<1.01$ \\
\hline $\begin{array}{c}\text { moderate or } \\
\text { mesoband }\end{array}$ & $1 \%<p b w \leq 100 \%$ & $1.01<b r \leq 3$ \\
\hline $\begin{array}{c}\text { ultramoderate } \\
\text { or } \\
\text { subhyperband }\end{array}$ & $100 \%<p b w \leq 163.6 \%$ & $3<b r \leq 10$ \\
\hline hyperband & $163.6 \%<p b w<200 \%$ & $b r \geq 10$ \\
\hline
\end{tabular}

\subsubsection{Classification based on technical attributes}

The possible EME created by IEMI sources are classified by the spectral content in [53]. A four-way categorization is made based on the frequency bandwidth of the source: narrow or hypoband, moderate or mesoband, ultramoderate or subhyperband, and hyperband. The categorization is defined by the bandratio $b r=f_{h} / f_{l}$, where $f_{h}$ is the upper frequency point and $f_{l}$ the lower frequency point. The frequency points are defined such that $90 \%$ of the signal energy is contained within these frequency points. The frequency bandwidth classification adopted from [53] is presented in Table 2.1. As an example, in [54] an overview is given of narrowband sources and in [55] an overview is given of wideband sources.

Three different waveforms can be distinguished that are common for HPEM; narrowband waveform, ultrawideband waveform, and a damped sinusoidal waveform [56]. An overview of these waveforms, both in time and frequency domain, are shown in Figure 2.2. Most waveforms of IEMI sources are similar to these waveforms or are a combination of them.

A narrowband waveform can emit a high amplitude burst of pulses at a carrier frequency, with each pulse containing many cycles, at a certain pulse repetition frequency (PRF), or a continuous signal. The majority of its energy is centered around a single frequency, i.e., the carrier frequency. The carrier frequency can be tuned to the vulnerable frequency to increase the chance of a successful attack, but this implies that the vulnerable frequency needs to be known a priori. In the case of wireless communication this can easily be determined, and the front door coupling can be maximized with a narrowband source tuned to the operating frequency of the communication system. A narrowband waveform can be described in the time domain 
by:

$$
a(t)=A_{0} \sin \omega_{0} t \cdot u(t)
$$

and in the frequency domain by:

$$
A(\omega)=-A_{0} j \frac{\pi}{2}\left[\delta\left(\omega-\omega_{0}\right)-\delta\left(\omega+\omega_{0}\right)\right]+\frac{\omega_{0}}{\omega_{0}^{2}-\omega^{2}} .
$$

In these equations, $A_{0}$ is the peak amplitude, $\omega_{0}$ is the angular centre frequency, and $u(t)$ is the Heaviside step function.

An UWB waveform, or hyperband, is represented by a double exponential pulse with very short rise time and short full-width-at-half-maximum (FWHM) time. Opposed to the narrowband waveform, this waveform spreads its energy over a very wide frequency band, resulting in a relatively low power density. Since an UWB covers a large frequency band, it is likely to cover a vulnerable frequency band of the victim system. However, as mentioned, the power density is relatively low, and the energy of a UWB pulse is very low because it is extremely short, which makes it less likely to cause damage to a system. An UWB waveform is described in the time domain by:

$$
b(t)=B_{0}\left(e^{-\alpha t}-e^{-\beta t}\right) \cdot u(t),
$$

and in the frequency domain by:

$$
B(\omega)=\frac{B_{0}(\beta-\alpha)}{(\alpha+j \omega)(\beta+j \omega)} .
$$

In these equations, $\alpha$ and $\beta$ are directly related to the rise-time and the FWHM of the waveform.

A damped sinusoidal waveform is a combination of the previous two waveforms; it has the short rise time of a UWB pulse and a centre frequency carrying a large part of the energy. Repetitive pulses of a damped sinusoidal waveform are called a dispatcher, which stands for damped intensive sinusoidal pulsed antenna. Dispatcher create highly energetic radiation and fall often in the mesoband category [5]. A damped sinusoidal waveform is described in the time domain by:

$$
c(t)=C_{0} e^{-\alpha t} \sin \omega_{0} t \cdot u(t),
$$

and in the frequency domain by:

$$
C(\omega)=\frac{C_{0} \omega_{0}}{(\alpha+j \omega)^{2}+\omega_{0}^{2}} .
$$

In these equations, $\alpha$ represents the damping factor of the oscillation.

The EME generated by the source can also be classified by the E-field strength at a specified distance, the frequency agility, the duration and repetition rates for 


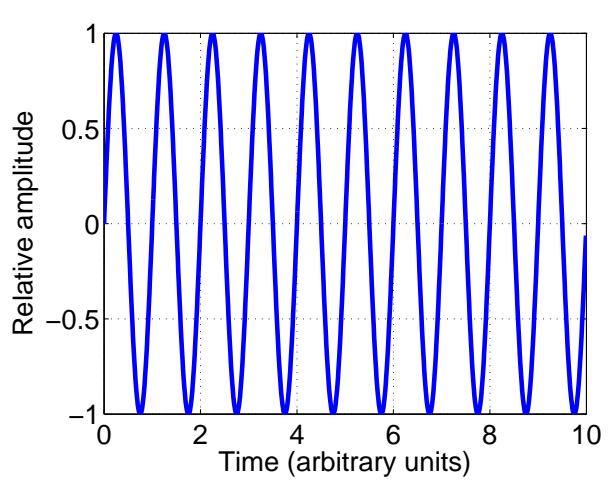

(a) Narrowband waveform.

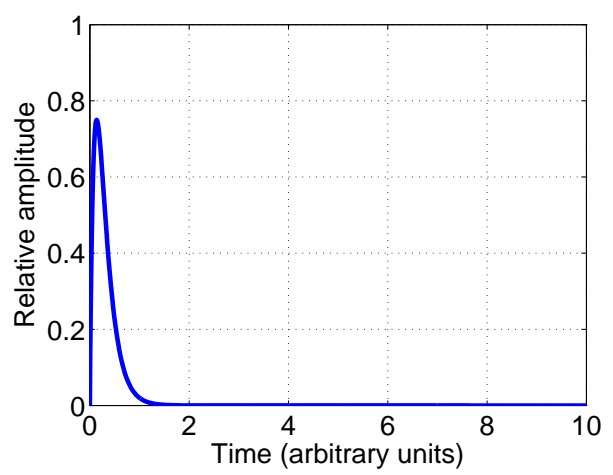

(c) UWB waveform.

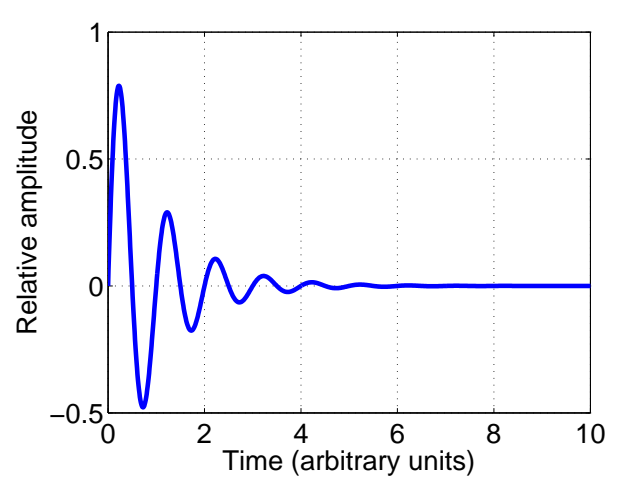

(e) Damped sinusoidal waveform.

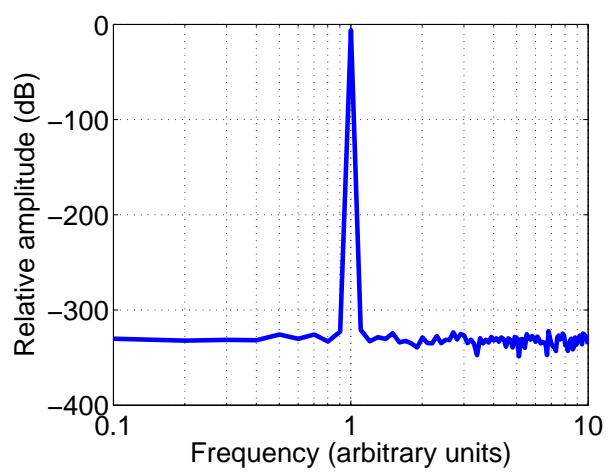

(b) Frequency content of the narrowband waveform.

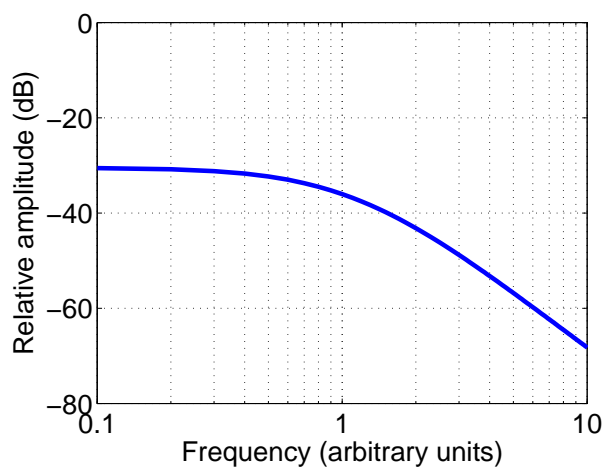

(d) Frequency content of the UWB waveform.

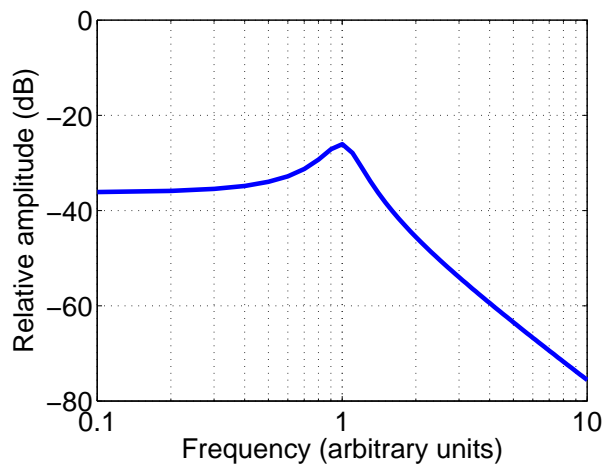

(f) Frequency content of the damped sinusoidal waveform.

Figure 2.2: Time and frequency description of the three different waveforms. 
pulsed sources, and the burst lenght [57]. Another commonly used figure of merit for defining HPEM sources is the far voltage, which is the product of the peak electric field (measured in the far field) and the distance between the source and location where the peak electric field is measured. In this way, it is easy to calculate the peak electric field generated by a HPEM source at a specified distance; this is simply the far voltage divided by the distance. All these technical parameters are influencing the effect an EME can have on a target system, i.e. the ability to cause a disruption.

\subsubsection{Risk potential of IEMI source}

As mentioned before, to analyse the risk a IEMI sources poses to a target system, it is not sufficient to take only technical attributes into account. As explained in [58], the risk is also dependent upon:

- Likelihood of occurrence of the EME;

- Ability to access the target system;

- Sensitivity of the target to the EME.

It is stated in [56] that the likelihood of occurrence of an EME in general decreases as the pulse energy of the EMI increases. This is graphically clarified by Figure 2.3 [56]. The rationale behind this reasoning is that a system that can deliver a pulse carrying a large amount of energy to the target system is most likely a highly sophisticated system with high cost and having a large size (so not very transportable). The ability to access a target system is dependent on both the portability of the IEMI source and the accessibility of the system. The accessibility of an infrastructure and the sensitivity of the target are further discussed in Section 2.4. To assess the risk potential of an IEMI source, classification will also be based upon source technology, portability, and availability.

\section{Source technology}

Different sources can be classified by their technical sophistication level in assembling and deploying such systems. The levels are in [5] divided into; low-tech, medium-tech, and high-tech generator systems.

Low-tech generator systems require minimal technical capabilities, possess marginal component performance, and are easily assembled and deployed while hiding behind dielectric truck walls or in similar vehicles. Med-tech generator systems require the skills of a qualified electrical engineer, have relatively more sophisticated components, and can be a modified commercially-available radar system. High-tech generator systems require specialized and sophisticated technologies, and may be specifically tuned to cause severe damage to specific targets. 


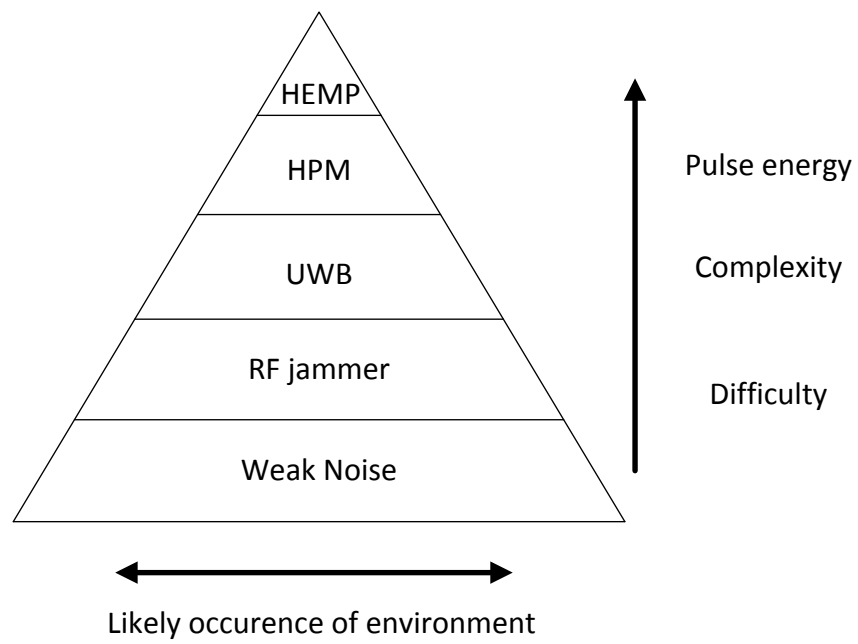

Figure 2.3: Likelihood of occurence for different EME.

\section{Portability}

The portability of the sources is divided into four different levels as described in [59]; pocket-sized, briefcase sized, motor-vehicle sized, and trailer sized.

In Table 2.2, the portability levels are defined. Level 1 applies to threat devices that can be hidden in the human body and/or in the clothing. Level 2 applies to threat devices that are too large to be hidden in the human body and/or in the clothing, but are still small enough to be carried by a person (such as in a briefcase or a backpack). Level 3 applies to threat devices that are too large to be easily carried by a person, but large enough to be hidden in a typical consumer motor vehicle. Finally, level 4 applies to threat devices that are too large to be either easily carried by a person or hidden in a typical consumer motor vehicle. Such threat devices require transportation using a commercial/industrial transportation vehicle.

Table 2.2: Definitions of portability levels as defined in [59].

\begin{tabular}{|c|c|}
\hline Portability level & Definition \\
\hline 1 & Pocket-sized or body-worn \\
\hline 2 & Briefcase or backpack-sized \\
\hline 3 & Motor Vehicle-sized \\
\hline 4 & Trailer-sized \\
\hline
\end{tabular}




\section{Availability}

Availability is a measure of both cost and the technological sophistication as described in [59]. Four different levels are classified ranging from 1 to 4 , where 4 means that the availability is low.

\subsubsection{Literature survey}

Throughout the literature many EM sources can be found that could potentially be considered as an IEMI threat. Within STRUCTURES 65 possible IEMI sources were classified according to their spectral attributes, field strength, source technology, portability, and availability. The results, from which general trends can be observed, are partly published by consortium partners in [6] and [57]. For instance, the survey clearly showed that with increasing field levels, the portability of the sources tend to decrease. Similarly, it was observed that sources which are highly available, produce lower field levels. The classification aids the understanding of the risk of a possible IEMI source. As an example, the risk of an IEMI source increases with a higher portability, since the ability to access the target system increases. Similarly for availability and source technology, i.e., low-tech generator systems with a high availability are more likely to be used as an IEMI source.

\subsection{Coupling of IEMI}

The coupling of EMI to a large complex and distributed CI is difficult to analyse. There are often many possible points of entry through which IEMI can couple to the system. The coupling paths can be both radiated and conducted and often the complete coupling path is a combination of both. Examples of coupling of conducted interference through possible points of entry (e.g. a power socket) are described in $[27,28]$. In these papers, the point of entry analysed is a power socket, which is normally not considered for high frequency or high power disturbances [60]. The EM waves can couple into the electronic systems through the front door or through the back door. These coupling methods are in [31] and [34] defined as:

- Front door coupling: The energy uses available ports intended for the propagation of electromagnetic energy and communication with the external environment, e.g., antennas or power sockets. This can cause interference in-band and/or out-of-band through the ports used for coupling.

- Back door coupling: The electromagnetic energy uses ports and paths generally not intended for communication with the external environment, e.g., through walls or small apertures, or coupled onto cables. 
A typical example of an IEMI attack scenario is depicted in Figure 2.1, illustrating both front door and back door coupling mechanisms. The coupling of radiated EM energy to a receiver comprises a number of factors. The emitted energy will be attenuated by the free space loss factor, i.e. the power density falls off as $1 / r^{2}$, with $r$ being the distance. Besides the free space losses, there are the atmospheric losses, which are dependent on weather conditions. Often, electronic equipment is located inside a building, and therefore the walls will cause another frequency dependent attenuation. As can be understood, it is complex to estimate the coupling from IEMI correctly to a critical subsystem of an infrastructure. Often measurements or simulations are needed to determine the transfer function from an IEMI source to a critical system of an infrastructure.

\subsubsection{Front door coupling}

Front door coupling of radiated interference is mostly via an antenna. Assuming far field conditions, the received signal power of the antenna equals [61]

$$
P_{\mathrm{rx}}=\frac{E^{2}}{Z_{0}} \frac{\lambda^{2}}{4 \pi} G(\theta, \phi)\left(1-|\Gamma|^{2}\right) e_{\mathrm{p}},
$$

where $E$ is the RMS value of the electric field at the antenna, $Z_{0}$ is the wave impedance, $\lambda$ is the wavelength, $G(\theta, \phi)$ is the gain of the antenna as a function of the polar and azimuthal angle, $\Gamma$ is the antenna reflection coefficient, and $e_{\mathrm{p}}$ is the polarization mismatch. The polarization mismatch factor equals

$$
e_{\mathrm{p}}=\left|\hat{\boldsymbol{\rho}}_{\mathbf{w}} \cdot \hat{\boldsymbol{\rho}}_{\mathbf{a}}\right|^{2}
$$

where $\hat{\boldsymbol{\rho}}_{\mathrm{w}}$ is the unit vector of the incoming wave and $\hat{\boldsymbol{\rho}}_{\mathbf{a}}$ is polarization vector of the receiving antenna. The $E$-field at the receiving antenna due to the IEMI source in free space can be described as:

$$
E=\sqrt{\frac{P_{\mathrm{EIRP}}}{4 \pi r^{2}} Z_{0}}
$$

where $P_{\text {EIRP }}$ is the effective isotropic radiated power by the IEMI source, and $r$ is the distance between the receiver and the IEMI source. The $P_{\text {EIRP }}$ is dependent on both the power of the IEMI source and the directivity of the source antenna.

From (2.7) and (2.9), we can make two important observations. Firstly, the received power is space-dependent and related to the antenna pattern of both the source and receiver. The maximum amount of energy is received if the direction of the interferer is along the boresight of the receiving antenna. This is one of the reasons why front door interference can be relatively easily achieved at a large distance. The gain of the receiving system can be used by the adversary to effectively couple IEMI into the system. 
Secondly, the received power is strongly frequency dependent due to the antenna reflection coefficient. An antenna is often designed such that the coefficient is below $10 \mathrm{~dB}$ for the desired frequencies, i.e. in-band frequencies. For out-of-band frequencies the reflection coefficient can be higher, resulting in less received power. However, antennas can be very broadband or can have more resonating frequencies with a low reflection coefficient.

\subsubsection{Back door coupling}

Back door coupling is more complex as compared to front door coupling. With front door coupling, the attacker often has knowledge of the coupling mechanisms - for instance the operating frequencies of the antennas - whereas with back door coupling this information is unknown or difficult to obtain. As illustrated in Figure 2.1, the coupling is complex and it is likely that exact locations of critical or vulnerable equipment is unknown.

There are several different coupling mechanisms that can play a role: conducted coupling, field-to-wire coupling, wire-to-field coupling, aperture coupling, and aspects such as reflection, diffraction, and absorption. In [21] Baum attempts to show how one can optimize the coupling of EM energy via a backdoor coupling at a distance.

In Figure 2.4, the system response as a function of frequency to incident EM waves is depicted [21]. As can be seen, there is a resonance region where the impact is maximized. This graph can be explained as follows: for higher frequencies (smaller wavelengths), the energy couples easier to the system interior through the seams, slots, apertures and other openings. However, with further increasing frequency, the fieldto-wire coupling decreases due to re-radiation losses and increasing path losses. These two opposing phenomena lead to the presented graph, where the electromagnetic response of the target system is maximized in the resonance region.

This resonance region is related to the wavelengths and is often estimated at 1 $\mathrm{GHz}$ to $3 \mathrm{GHz}$. Wavelengths in this frequency region (30 to $10 \mathrm{~cm}$ ) are comparable to the size of many electronic devices, such as cell phones and laptops, and therefore the coupling is maximized.

\subsection{Critical infrastructures}

The risk IEMI poses to a facility or critical infrastructure (CI) is not easy to quantify. Again, for a full risk assessment of IEMI one has to look at both technical attributes and non-technical attributes. In the next section, a classification methodology for facilities with respect to IEMI is described. This section is a review of the work of Mansson et al. in [62]. 


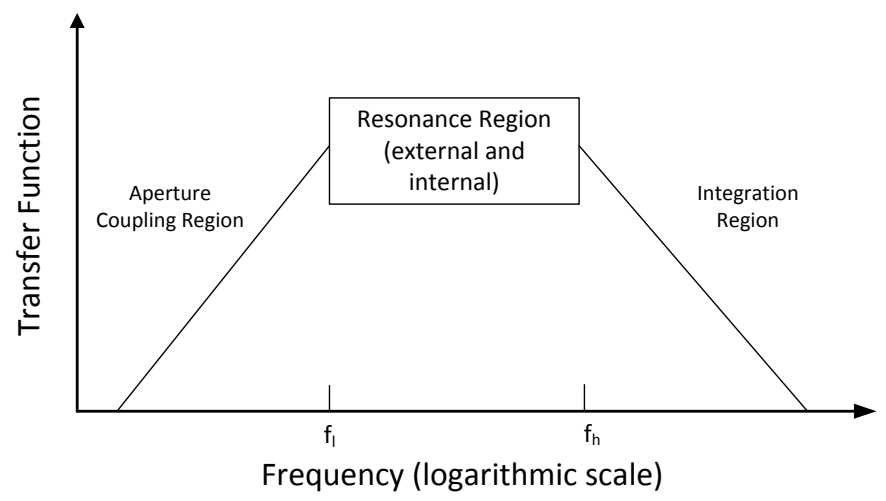

Figure 2.4: System response as a function of frequency. Adopted from [21].

A classification of the vulnerability of facilities based on the accessibility, susceptibility, and consequence (ASC) is proposed in [62]. The contributions of these three aspects to a systems hardness against IEMI is clarified in Figure 2.5. Essentially, the quantified ASC is represented by a vulnerability vector with a good hardness near the origin and a bad hardness in the farthest corner of the ASC cube.

\subsubsection{Accessibility}

The accessibility of a system describes the ability to gain access to the different parts of the facility or the ability to get in close vicinity of critical components of the facility. For system hardness a low accessibility is desired. The scaling of the accessibility should be qualitatively measured and the meaning, the number of levels, and the differences of these degrees have to be clarified.

By application of the electromagnetic topology (EMT) approach, a facility or infrastructure can be divided into various EM zones [63]. For large infrastructures, these various zones can be for instance different building or rooms and each EM zone can have a different level of accessibility. The EM coupling from one zone to another is often represented by a transfer function; for instance coupling from outside to inside a building.

It is useful to transform a facility into an EMT diagram and number the zones hierarchically. The accessibility is expected to vary with zone number and decreases with increasing zone number, i.e., the zone numbers are ordered from outer zones to inner zones and inner zones should be less accessible. The accessibility of zones can be approved by having access control. Some facilities have guard control, where access rights are needed to enter the building, which greatly lowers the accessibility. 


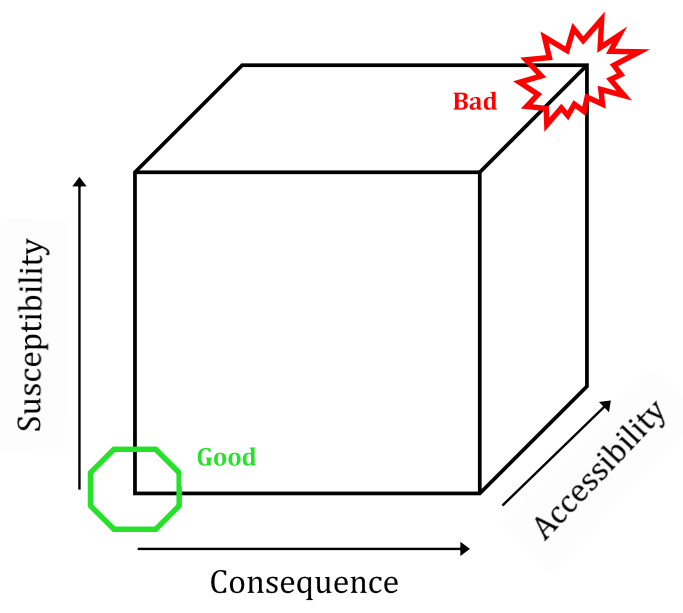

Figure 2.5: Risk cube dependent on three quantities that can be used to analyse the IEMI hardness of a system. Adopted from [62].

\subsubsection{Consequence}

With consequences the result of a successful IEMI attack on a CI is meant and it is best determined by the system owner or operator. The consequence also depends on the interdependency with other infrastructures, as is described in [3]. The scaling can be qualitatively measured, in a similar qualitative way as the scaling of the accessibility is determined.

The consequence of a system mishap is in MIL-STD-882E [64] classified by various severity categories. The severity categories presented in [64] are summarized in Table 2.3 .

\subsubsection{Susceptibility}

Susceptibility is defined in [5] as:

"inability of a device, equipment or system to perform without degradation in the presence of an electromagnetic disturbance"

Essentially, this is a technical aspect of a system that has been often evaluated by EMC engineers.

For a large, complex, and distributed system, this term has to be reviewed. The susceptibility of such a system is also dependent on the tolerance of the facility against faults (redundancy), and on the ability to handle, or mitigate, disturbances. Of course, it is still based on the susceptibility of its components in terms of electric fields, induced current, and voltages, but this is not sufficient. 
Table 2.3: Severity categories as defined in [64].

\begin{tabular}{|c|c|l|}
\hline $\begin{array}{c}\text { Descrip- } \\
\text { tion }\end{array}$ & $\begin{array}{c}\text { Severity } \\
\text { Category }\end{array}$ & Mishap Result Criteria \\
\hline Catastrophic & 1 & $\begin{array}{l}\text { Could result in one or more of the following: death, } \\
\text { permanent total disability, irreversible significant } \\
\text { environmental impact, or monetary loss equal to or } \\
\text { exceeding } \$ 10 \mathrm{M} .\end{array}$ \\
\hline Critical & 2 & $\begin{array}{l}\text { Could result in one or more of the following: } \\
\text { permanent partial disability,injuries or occupational } \\
\text { illness that may result in hospitalization of at least } \\
\text { three personnel, reversible significant environmental } \\
\text { impact, or monetary loss equal to or exceeding } \$ 1 \mathrm{M} \\
\text { but less than } \$ 10 \mathrm{M} \text {. }\end{array}$ \\
\hline Marginal & 3 & $\begin{array}{l}\text { Could result in one or more of the following: injury } \\
\text { or occupational illness resulting in one or more lost } \\
\text { work day(s), reversible moderate environmental } \\
\text { impact, or monetary loss equal to or exceeding } \\
\$ 100 \mathrm{~K} \text { but less than } \$ 1 \text { M. }\end{array}$ \\
\hline Negligible & $\begin{array}{l}\text { Could result in one or more of the following: injury } \\
\text { or occupational illness not resulting in a lost work } \\
\text { day, minimal environmental impact, or monetary } \\
\text { loss less than } \$ 100 \mathrm{~K} .\end{array}$ \\
\hline
\end{tabular}

The system may be built in such a way, that it automatically reconfigures itself, even though some subsystems or components are disturbed by IEMI. As a result the susceptibility of a system cannot be simply defined in physical parameters, e.g., volts per meter.

In analysing the susceptibility of a CI, it is important to identify the critical subsystems, i.e., a subsystem that is critical for the functioning of an infrastructure. As an example, the GPS system in a plane is useful, but it is not critical, because there is other instrumentation that enables navigation. In other words, we can again look at the consequence of failure of this subsystem. Once the critical subsystems are identified, we can look into their electronic components and there susceptibility levels. These susceptibility levels can be determined either from literature, simulation or measurements. 


\subsection{Wireless communication infrastructure}

In the next section, an overview is presented of a typical wireless infrastructure. Then a qualitative analysis will be presented of the risk IEMI poses to wireless communication, using the non-technical attributes presented in this chapter.

\subsubsection{Overview of a typical wireless instrastructure}

The majority of the wireless communication infrastructures is based on the cellular principle. In a cellular radio network the geographical area is divided into cells, with each cell being served by at least one base station transceiver. It offers great advantages such as the possibility for a high number of users through the limited allocated spectrum, possibility for a wide coverage area, and low power restraints on the terminal equipment [65].

A system overview of a cellular system is presented in Figure 2.6. The terminal equipment is connected to the access network via a wireless user-network interface (UNI). Terminal equipment can be all sorts of devices, such as cell phones, laptops, or even cars. The access network consists of the base station and the base station controller.

The base station communicates directly with the end users, and are most often situated at a tall tower or building. Each base station can facilitate many end users within one cell. The communication between the base station and the base station controller is mostly over fibre. Base station controllers are connected to a multitude of base station and forward the received data to the mobile switching center. From there on the data is distributed over the transit network to the access network of the end connection.

\subsubsection{Analysis of the IEMI threat for wireless communication}

As mentioned in Chapter 1 and Chapter 2.3.1, wireless communication itself is vulnerable against IEMI because of the open access nature of the wireless medium and the easy point of entry via the antennas. The critical parts of a wireless infrastructure are the wireless link and the involved subsystems, i.e., the receivers of the base station and the terminal equipment.

Protection of these critical subsystems is not easy, because the infrastructure is widely distributed and easily accessible; the locations of most base stations are publicly accessible and are not fenced. Besides this, the EMI is front door coupled using the antenna gain of the victim so it can be done at a large distance.

The base station needs additional attention in this analysis. As described in [66], it is most economical for an adversary to disrupt the base station, because this systems is at a fixed location and it is easy to get into line-of-sight of the receiving antenna. 


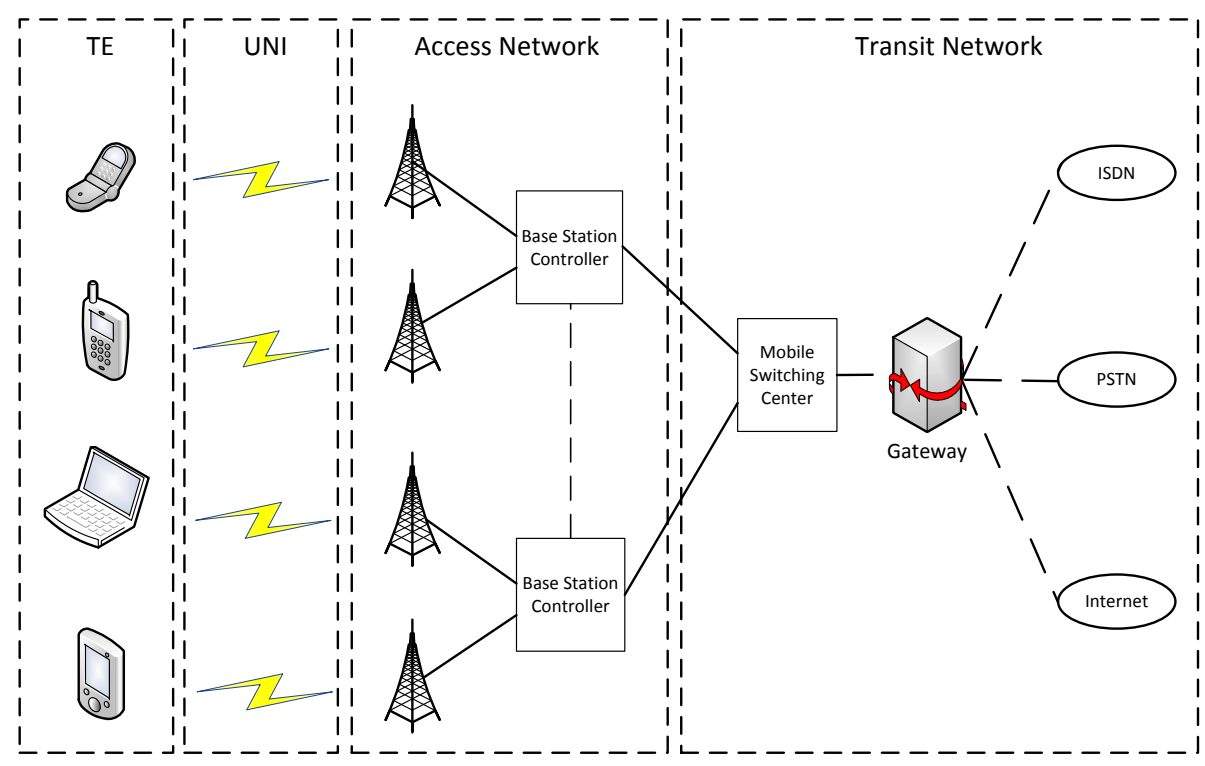

Figure 2.6: Reference configuration of a wireless communication infrastructure.

A mobile device can be moved to diminish the impact of interference or to prevent line-of-sight. The fixed position of the base station enables a jammer to be in close proximity of the base station giving it a power advantage over the terminal equipment. The consequence of disrupting a base station is also larger, since a complete cell will be denied communication services.

From this short analysis, it is easily concluded that the wireless infrastructure is vulnerable to IEMI. However, as explained in [67], it is also necessary to assess the likelihood of an IEMI event to give a realistic risk assessment. The question that should be asked is whether it is likely that an adversary conducts an IEMI attack against a wireless system.

To this end, the likelihood can be classified by: 1) availability of an IEMI source, 2) required knowledge for an attack, and 3) the cost of an attack.

Typical IEMI sources that can disrupt wireless communication are RF jammers. The risk potential of these sources are high according to Section 2.2.2. These jammers are widely available and can easily be purchased online well below $\$ 500$. The source technology and required knowledge is minimal, i.e. in the description it is stated what communication systems it is capable of disrupting and within what distance. Besides this, the sources are highly portable and easily brought into close vicinity of victim systems.

So it can be concluded that IEMI poses a serious threat to wireless communication 
and that additional research is required to get a better insight into the vulnerabilities and to identify protection strategies. This conclusion is supported by the account of numerous IEMI attacks against various wireless communication systems [68-71].

\subsection{Summary and conclusions}

In this chapter, an overview is presented of the threat related to an IEMI attack, involving both technical and non-technical attributes. A risk analysis would start with analysing the susceptibility of a CI by identifying the critical subsystems and their susceptibility levels. Once the levels are known, a risk estimate can be made by combining knowledge on available IEMI sources, possible coupling paths, and the accessibility of the infrastructure.

It can be concluded that IEMI can be a serious threat for wireless communication and serious efforts should be taken to minimise this risk. The wireless link and receivers were recognized to be the most vulnerable and critical subsystems of the wireless infrastructure. In the remainder of this thesis, the focus is on the susceptibility of a wireless link and the involved receivers, and on possible techniques to improve the system's robustness against IEMI. In the next chapter the susceptibility levels of wireless communication will be thoroughly investigated, and relevant interference mechanisms will be identified. 


\section{Chapter 3}

\section{Interference mechanisms}

The susceptibility levels of a wireless link and wireless receiver against IEMI will be analyzed in this chapter. The results presented in this chapter are published in the IEEE Transactions on Electromagnetic Compatibility [72] and are presented at the European Electromagnetics Symposium 2016 [73].

\subsection{Rationale}

The focus is only on front door coupled interference in this chapter, since this is recognized as the critical coupling mechanism in the previous chapter. Results of a susceptibility analysis are important to estimate the vulnerability of a wireless communication infrastructure against IEMI.

The qualitative analysis is applicable to any wireless network, but quantitative results will be presented specifically focused on Terrestrial Trunked RAdio (TETRA). TETRA is a digital standard that was developed by the European Telecommunication Standards Institute (ETSI) to meet the needs of professional mobile radio (PMR) [74], and as such is considered to be a critical infrastructure. TETRA is designed to be robust, and therefore we chose to further investigate this system.

The outline of this chapter is as follows. An overview of general wireless receivers is given in the next section. In Section 3.3, a brief summary of the TETRA air interface is given and a typical TETRA base station is presented. In Section 3.4, the different interference mechanisms are identified and discussed. In Section 3.5 and 3.6, an experimental analysis of a TETRA base station is presented. Finally, in Section 3.7 , a discussion on the analysis and results is presented. 


\subsection{Overview of typical wireless receiver}

The front end of a receiver is directly connected to the antenna, and is therefore the critical part to be investigated. An overview of a common receiver architecture is shown in Figure 3.1. The RF front end includes the front door filter, low noise amplifier (LNA), and the mixer. The received signal is fed to the LNA via a front door filter, which will amplify the signal with a very low noise contribution. Next, the mixer converts the amplified signal to a lower intermediate frequency (IF). At lower frequencies it is easier to implement a sharp analog filter to suppress out-of-channel interference, and thus to achieve a high selectivity. Finally, the signal can be amplified again and will be converted to the digital domain for further processing.

The selectivity of a receiver can be defined as the ability of a receiver to correctly detect and decode a desired signal in the presence of unwanted signals nearby in frequency. Selectivity is achieved by implementing high quality filters that attenuates all other frequencies except for the desired channel. The channel-selection filtering is being done at lower IF frequencies, since the required value of $\mathrm{Q}$ is then much lower. It should be clear that all of the stages in the receiver preceding the channel selection filter should be sufficiently linear to avoid non-linear effects, such as compression and intermodulation, resulting from unwanted spectral components.

Often there is a front door filter installed directly after the antenna. The function of this filter is to select an entire band and suppress the out-of-band (OOB) interferers. So it protects the receiver against unwanted effects of OOB interferers, but not against in-band interferers. The filter is not very selective and is not present in every receiver. The insertion loss of a filter reduces the noise performance of the receiver - and as a result the sensitivity - and for this reason designers might decide not to include them.

The noise performance of a wireless receiver is one of the key characteristics. Both passive and active components in a receiver are noisy and this leads to a degradation in signal-to-noise ratio (SNR). The ratio between the SNR at the output of a receiver over the SNR at the input receiver is defined as the noise figure. To calculate the noise figure of an cascaded system such as a receiver, we need to know the noise figure of each individual stage in the receiver chain. The total noise figure of a receiver can be expressed by [65]:

$$
F_{\mathrm{tot}}=F_{1}+\frac{F_{2}-1}{G_{1}}+\frac{F_{3}-1}{G_{2}}+\ldots
$$

where $F_{i}$ and $G_{i}$ are the individual noise figures and noise gains of the subsequent stages in a receiver. From the Friis formula it is obvious that the first stage needs special attention. From a functional point of view it is preferred that the first stage has a low noise figure and a high gain. This is the reason the first stage of a receiver is a LNA. A high gain in the first stage suppresses the noise contribution of subsequent stages. 


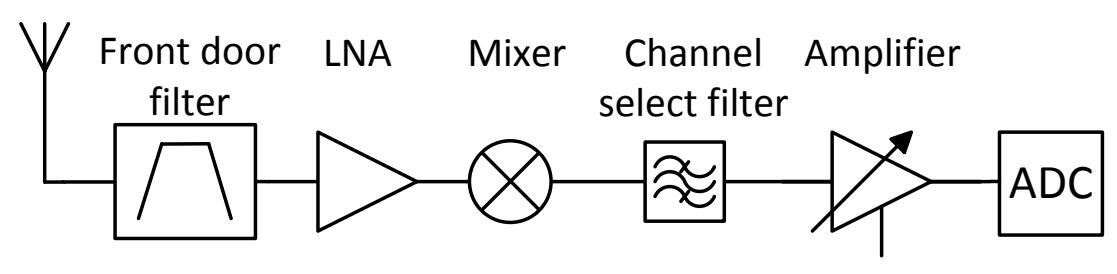

Figure 3.1: Typical receiver architecture.

\subsection{TETRA overview}

A variety of mission-critical communication applications are based upon TETRA such as: public safety, transportation, government, utilities, military, and more. For these type of applications, it is crucial that the system continues to function, even under harsh conditions. More information on the TETRA protocols can be found in $[75,76]$. A brief overview of the TETRA air interface as used by the emergency services in the Netherlands, and the structure of a typical base station, is described in this section.

\subsubsection{Air interface}

The air interface for the TETRA system is defined in [77]. It contains the specifications of the physical layer, the data link layer and the network layer according to the OSI model. The system can work in two different modes; the direct mode and the trunked mode. In the direct mode the mobile stations (MS) communicate directly with each other, whereas in the trunked mode it communicates via a base station (BS). In trunked mode operation, TETRA is comparable to any cellular communication system, e.g. GSM, but there are some major differences [76]:

- Security, the traffic data can be encrypted, and terminal equipment have a TETRA Equipment Identification for registration and authentication;

- Group calls, one-to-many, and many-to-many connections are possible;

- Fast call set-up, TETRA users with a push-to-talk set up a connection without noticeable delay;

- Capacity, in TETRA the data transfer is rather slow compared to modern standards, partly because more priority is given to robustness than to optimization for capacity. 
The TETRA system can operate in the bandwidth of $100 \mathrm{MHz}$ up to $900 \mathrm{MHz}$ [78]. The emergency services in the Netherlands operate in the 380 to $395 \mathrm{MHz}$. The access scheme is time division multiple access (TDMA) [65] with 4 channels per carrier. A physical channel is defined for a specific carrier frequency and a specific TDMA slot number. The physical channel can be a control channel or a traffic channel. The carrier data rate is $36 \mathrm{kbit} / \mathrm{s}$ and the user data rate is $7.2 \mathrm{kbit} / \mathrm{s}$ per time slot. The most used modulation scheme is $\pi / 4$-shifted differential quaternary phase shift keying ( $\pi / 4$-DQPSK). More information on $\pi / 4$-DQPSK can be found at $[65,79]$. The carrier spacing is $25 \mathrm{kHz}$. There is a fixed frequency separation between the downlink and the uplink. The dynamic reference sensitivity of the MS and BS are respectively -103 $\mathrm{dBm}$ and $-106 \mathrm{dBm}$ and power levels as strong as $-20 \mathrm{dBm}$ can correctly be received at the BS [77]. So it can be concluded that the dynamic range of the BS receiver is over $80 \mathrm{~dB}$.

The data link layer on top of the physical layer provides error free communication. It adds error detection and error correction. The number or rate of bit errors that is acceptable for error free communication is dependent on the type of error control scheme implemented in the data link layer [77].

\subsubsection{Typical base station structure}

It is important to note that for TETRA only the air interface is described. Used equipment, such as base stations, can be manufactured in a specific way as long as the equipment complies with the minimum technical characteristics as described in [80]. A typical structure of a base station receiver as is often seen in the Netherlands is described in this section.

Typically in the Netherlands, TETRA base stations are equipped with three vertical polarized dipoles. The three signals are summed and connected to a single input of the receiver. A typical structure of the receiving part at a TETRA base station tower is given in Figure 3.2. This is the structure of the base station receiver that will be analyzed in Section 3.6. The combined signal from the antennas has a coaxial connection to a cavity band pass filter (BPF). Next, the received signal is connected with a coax cable to a low noise block (LNB) containing a LNA and a splitter. The LNB has 4 equal outputs that are fed to the base station. Every single output of the front end can be processed individually by the base station. In the base station, the signal is filtered and converted to a $70 \mathrm{MHz}$ intermediate frequency. Next, the 70 $\mathrm{MHz}$ signal is converted to the digital domain for further processing. The receiver is based on the heterodyne principle, i.e., the received signal is first down converted to an IF to improve channel filtering [81].

The scattering parameters (S-parameters) of a BPF, which is used at TETRA base stations, were measured with a vector network analyzer (VNA) and the results 


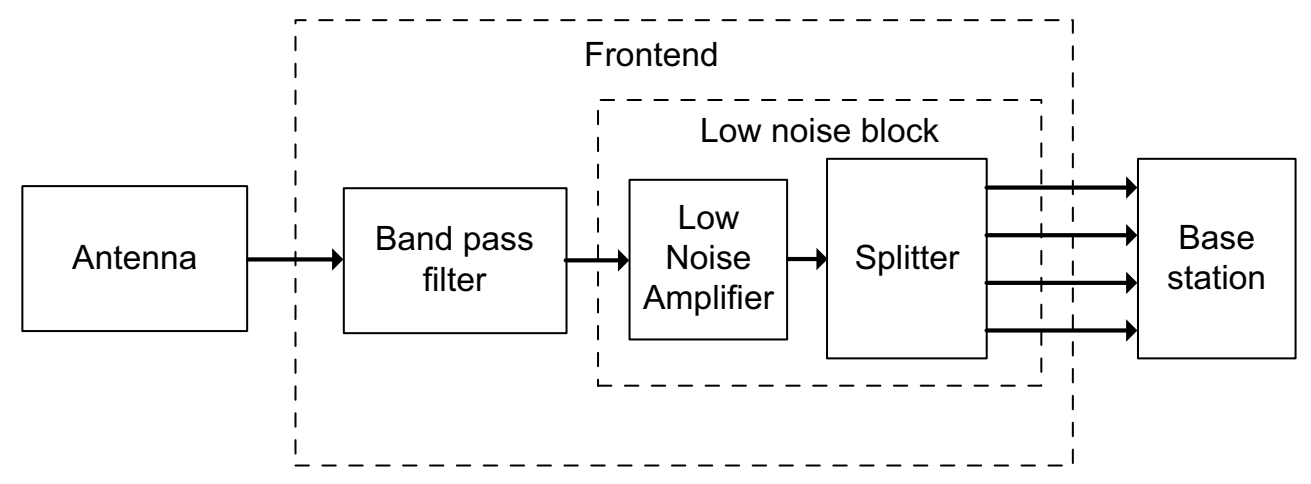

Figure 3.2: Typical structure of the receiver at a TETRA BS tower.

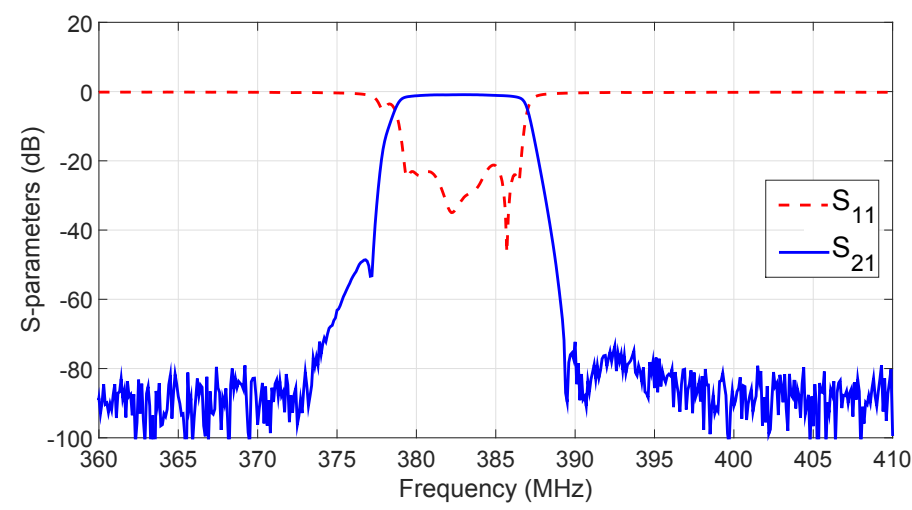

Figure 3.3: S-parameters of a BPF as a function of frequency.

are presented in Figure 3.3. It can be seen that this BPF is a pass band from approximately $380-385 \mathrm{MHz}$ and the OOB interference is $80 \mathrm{~dB}$ attenuated. The transition bandwidth from pass band to stop band is approximately $6 \mathrm{MHz}$. This high quality filter is a bulky cavity filter which is very suitable for base stations, but not for terminal equipment due to the form factor. Typically, at terminal equipment, such as cell phones, the front end filter is a surface acoustic wave (SAW) filter [82].

\subsection{Analysis on front door coupled IEMI}

In this section, the different interference mechanisms of front door coupled IEMI on the previous discussed TETRA base station will be analyzed. It should be clear that this analysis can be extended to any wireless receiver.

To understand the various interference mechanisms it is important to analyze the frequency content of the radiated IEMI. Front door coupled IEMI is defined to 
be in-band if the frequency is within the pass band of the front end filter or of the antenna. If not, the interference is defined to be OOB. The front end BPF of a TETRA base station often has a bandwidth of $5 \mathrm{MHz}$, but the bandwidth of a TETRA communication channel is only $25 \mathrm{kHz}$. This means that in-band interference can be both in-channel or out-of-channel. The effect of out-of-channel interference on the performance of a receiver depends on its selectivity. In Figure 3.4, the different frequency domains of IEMI of a wireless receiver are graphically shown. Of course, wideband interference can spread its energy over several frequency domains. In case of wide band interference, the total power at the input of the LNA can be approximated by integrating the power density of the interference over the bandwidth of the front end filter.

The goal of IEMI at the receiver is disrupting the detection of communication signals resulting in a DoS of the system. As described in [22], an IEMI attack can be classified by the physical mechanism causing the detrimental effect. We can recognize three different interfering mechanisms of front door coupled IEMI that can result in a DoS. Firstly, interference can damage the receiver leading to a permanent DoS. Secondly, interference can saturate the receiver resulting in a desensitization of the receiver. Thirdly, interference can mask the communication signal such that the receiver is unable to properly detect the received signal. This jamming of the communication signal can be done in a crude way or using intelligent techniques. Intelligent jamming techniques make use of vulnerabilities of the systems higher layer protocols making the jamming attack power efficient and hard to detect. Damage or saturation of a wireless receiver can only be achieved with high-power EMI, i.e., power levels greatly exceeding the normally received signal power. Masking the communication signal can already be achieved with power levels comparable to the signal power level. Protection strategies against the different interfering mechanisms are fundamentally different and need a separate analysis.

\subsubsection{Damage}

As discussed in Section 3.2, typically the first active component in a wireless receiver is the LNA and for this reason the most susceptible component. High-power interference can result in permanent degradation of the LNA, or it can burn out. In [83], it is shown that a LNA - after suffering from permanent physical damage - is completely useless, resulting in a DoS of the system. A possible protection strategy against physical damage is by mounting a limiter before the LNA. The limiter will shunt away the peak current if it exceeds a certain threshold and so protecting the LNA from damage. It is important to notice that when a limiter-protected-receiver is subject to high-power EMI the receiver is not able to function, but at least no permanent damage will occur. 


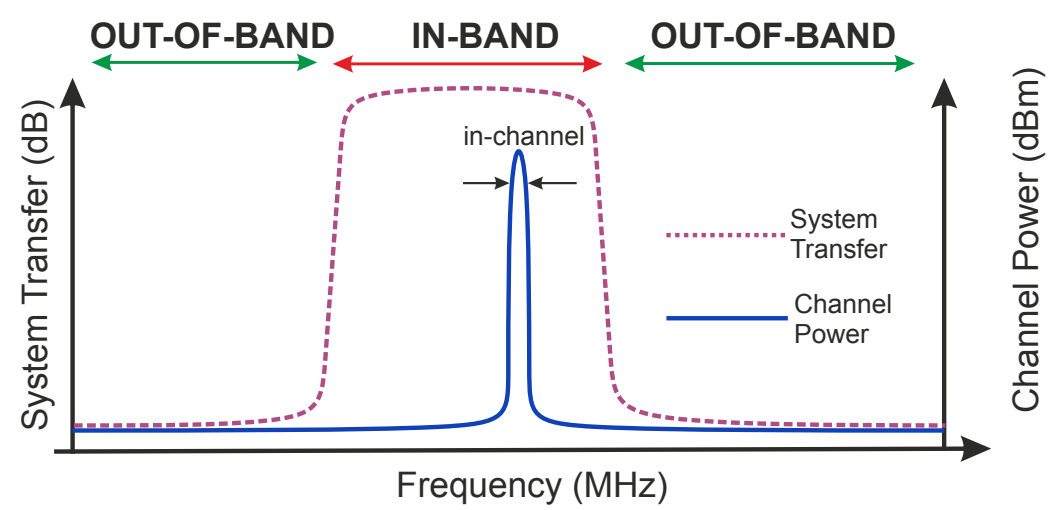

Figure 3.4: Graphical representation of the system transfer (from antenna to the LNA) and the channel power, showing the different frequency domains for IEMI.

In [83-85], extensive research has been presented on the destruction/ susceptibility levels of LNAs and on front door protection devices, i.e., limiters. In-band EM pulses with different pulse widths were injected into the LNAs designed to operate in C-band (4-8 GHz). Even though these LNAs operates at different frequencies than TETRA, meaning results are not directly comparable, the LNA technology (GaAs MESFET MMIC) of the samples was chosen to be general. For this reason, these thresholds are suitable to approximate destruction threshold levels for general LNAs working in a different frequency band. The destruction levels of the LNAs are summarized in [34] to be $34 \mathrm{dBm}$ for narrowband interference with a center frequency of $6 \mathrm{GHz}$ and a pulse width exceeding $1 \mu \mathrm{s}$. However, it has to be mentioned that it is very difficult to determined damage levels in a deterministic way. For instance, in a technical report from 1992 [86] the authors reported variations of damage levels of the same LNA - only different batch-between 3 and 15 Watt.

In [87], an analysis is presented on the survivability of GaN-based LNAs. It is well known that GaN technology transistors are outstanding for high-power applications, including robust LNAs with low noise figures. There are reports of LNAs surviving over $40 \mathrm{dBm}$ of input power continuously [88]. This technology is able to improve the robustness of a wireless receiver against damage. The disadvantage of GaN technology is currently the high costs involved. Both the material and the production process to make the devices are costly.

It is for now assumed that the damage threshold level of an LNA is $34 \mathrm{dBm}$. The base station antennas used by public safety sector national TETRA networks in The Netherlands have a omnidirectional gain of $8 \mathrm{dBi}$ [89]. Assuming a wavelength of $75 \mathrm{~cm}$ and free space wave impedance of $377 \Omega$, an E-field of $58 \mathrm{~V} / \mathrm{m}$ will result in a received power of $34 \mathrm{dBm}$. In [5], it is stated that it is possible to exceed E-fields of $100 \mathrm{~V} / \mathrm{m}$ at distance at a kilometer distance with commercially available sources, with 
modest sized antennas. A more detailed description on the susceptibility of wireless receiver against damage will be treated in Chapter 7 .

The investigated base station in this chapter is equipped with a BPF as a front door filter, see Figure 3.3. As can be seen, OOB interference is $80 \mathrm{~dB}$ attenuated, so it is safe to state that OOB interference is not likely to damage the LNA of the base station. To damage the receiver, the interference needs to be in-band, but it can be both in-channel or out-of-channel (see Figure 3.4). Most base stations are not equipped with a limiter, so this makes them vulnerable against high power in-band EMI that can damage the receiver.

In this analysis, we only focused on the damage level of the LNA and did not mention the $\mathrm{BPF}$ preceding the LNA. In case the BPF gets damaged, it might not provide the $80 \mathrm{~dB}$ attenuation for $\mathrm{OOB}$ interference anymore. Subsequent OOB interference could then possibly propagate into the LNA. A typical TETRA base station, and the base station we analyzed, have a RF cavity filter. The dominant mechanisms that cause a disruption in these type of filters is electrical breakdown inside the cavity or thermal-related high-power breakdown and detuning [90]. Power requirements to damage the filter would be very high as compared to damaging the LNA. For this reason, we did not further investigated this.

\subsubsection{Saturation}

Receiver components, such as the LNA or the mixer, can saturate for high input signals. For increasing input power, the receiver will first go into compression before actual damage will occur. For large input signals, a RF receiver becomes saturated and therefore non-linear. A saturated receiver will result in spurious cross modulation and intermodulation products, meaning the input spectrum is distorted.

Saturation of a system will often lead to compressive behavior, i.e., a decreasing gain for increasing input amplitude. This effect can be quantified by the 1-dB compression point, $P_{1-\mathrm{dB}}$, defined as the input signal level that causes the gain to decrease by $1 \mathrm{~dB}$ [81]. In Figure 3.5, $P_{1-\mathrm{dB}}$ is graphically explained. At the $1-\mathrm{dB}$ compression point, the output power is $1 \mathrm{~dB}$ lower than the output power expected from the theoretical response. The effect of gain compression is larger with amplitude modulation than with phase modulation. Gain compression only affects the amplitude of the received information signal, and the amplitude contains the information with an AM modulation scheme.

A high-power interferer, accompanying a desired signal, could saturate the receiver and, as a result, lower the SNR at the output of the receiver. SNR is a key characteristic in communication since it determines the sensitivity, i.e., the lowest detectable signal. The phenomenon of a desired signal superimposed on a large interferer experiencing gain reduction is called desensitization. A detailed investigation 


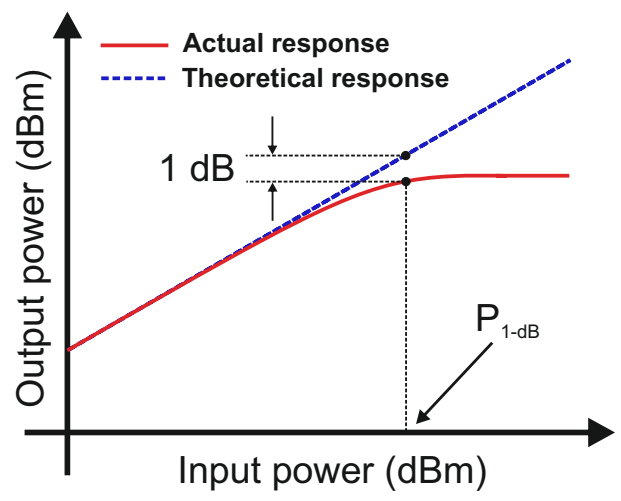

Figure 3.5: Graphical representation of $P_{1-\mathrm{dB}}$. The output power is plotted as a function of the input power.

on desensitization is presented in [91]. In this section, the focus is on the gain reduction of the desired signal due to an accompanying high-power interference or a blocker. This effect will be quantified by $P_{O B}$ defined as the input signal level of the blocker that causes the gain of the desired signal to decrease by $1 \mathrm{~dB}$. This wellknown phenomena can be modelled by a memoryless system with an input-to-output characteristic approximated by using a Taylor expansion:

$$
y(t) \approx \alpha_{1} x(t)+\alpha_{2} x^{2}(t)+\alpha_{3} x^{3}(t),
$$

where $y(t)$ is the output signal, $x(t)$ the input signal, and $\alpha_{1}, \alpha_{2}$, and $\alpha_{3}$ are the coefficients. Now assume $x(t)=V_{1} \cos \omega_{1} t+V_{2} \cos \omega_{2} t$, where the first term represents the desired signal, and the second term the interferer. If we substitute this in Eq. (3.2), and assume $V_{1} \ll V_{2}$, the output at frequency $\omega_{1}$ appears as

$$
y(t)=\left(\alpha_{1}+\frac{3}{2} \alpha_{3} V_{2}^{2}\right) V_{1} \cos \omega_{1} t .
$$

Assuming $\alpha_{1} \alpha_{3}<0$, it is easy to see from (3.3) that the gain experienced by the desired signal is a decreasing function of $V_{2}$. This desensitization lowers the SNR at the receiver output, e.g., the noise contribution of the following baseband blocks is increased. For a sufficiently large $V_{2}$, the gain can even drop to zero and the desired signal is completely blocked. Another important phenomenon that can come with a strong interferer accompanying a desired signal is called cross modulation. From Eq. (3.3) it can be easily seen that if the amplitude of $V_{2}$ is time varying, this variation can be seen in transfer of the desired signal at $\omega_{1}$.

To prevent the receiver from saturating, it requires a high dynamic range, such that it can still properly receive a very small signal, and at the same time a very 
strong signal can still be accurately processed. A TETRA base station is required to correctly receive signals with power levels up to $20 \mathrm{dBm}$ [77]. It is important to understand that IEMI can saturate the receiver both in-channel and out-of-channel, but if it is OOB it will be suppressed by the antenna transfer and the filter.

\subsubsection{Jamming}

Low-power interference cannot saturate or damage the receiver, however, it can mask the desired signal by decreasing the SNR. If the SNR is too low the receiver is not able to correctly detect the bits, and the resulting bit errors will disrupt the communication. This type of attack on wireless communication is generally referred to as jamming. There are many different jamming techniques and these techniques have been extensively studied [92-97].

An important measure to quantify a jamming attack is the jamming-to-signal ratio (JSR), which is given by the following equation [92]:

$$
\frac{J}{S}=\frac{P_{j} G_{j r} G_{r j} R_{t r}^{2} L_{r} B_{r}}{P_{t} G_{t r} G_{r t} R_{j r}^{2} L_{j} B_{j}}
$$

where

$P_{j}=$ Jammer power;

$P_{t}=$ Desired signal power;

$G_{j r}=$ Jammer antenna gain in the direction of the receiver;

$G_{r j}=$ Receiver antenna gain in the direcion of the jammer;

$G_{t r}=$ Transmitter antenna gain in the direction of the receiver;

$G_{r t}=$ Receiver antenna gain in the direction of the transmitter;

$R_{t r}^{2}=$ Distance between transmitter and receiver;

$R_{j r}^{2}=$ Distance between jammer and receiver;

$L_{r}=$ Jammer signal loss due to attenuation such as polarization loss;

$L_{j}=$ Transmitter signal loss due to attenuation such as polarization loss;

$B_{r}=$ Bandwidth of the receiver system;

$B_{j}=$ Bandwidth of the jamming signal.

It is assumed here that $B_{j}>B_{r}$. Usually a successful jamming attack requires the jamming power to be roughly equal to the signal power.

Digital communication often increases the robustness against errors by employing techniques such as coding and interleaving. Therefore digital communication is robust against low SNR values, but only to some extend. All in all, the effectiveness of a jamming attack depends on many parameters such as the JSR, modulation scheme, channel coding, and interleaving of the target system [66]. The primary measure to quantize the performance of a digital communication system is the bit-error-ratio 
(BER) [98]. It is typically a non-linear function of the SNR, or, in the case of dominant interference, of the signal-to-interference ratio (SIR). The BER of a data stream is defined as

$$
\mathrm{BER}=\frac{\text { Bit Errors }}{\text { Total Number of Transmitted Bits }}
$$

The data stream in TETRA includes redundancy to correct errors, but for a critical BER the base station will not be able to correct the errors and the reception is disturbed. The critical BER is dependent on the error code scheme that is implemented and can be different from system to system [75]. The BER is dependent on the detector scheme implemented in the receiver. A comprehensive description of the BER of $\pi / 4$-DQPSK modulated signal with a differential detection scheme is given in [79]. In the testing specification standard of TETRA [80], it is stated that a base station should be capable of receiving a $-112 \mathrm{dBm}$ signal with a BER below $3.66 \%$ in the presence of a $-25 \mathrm{dBm}$ interferer at $1 \mathrm{MHz}$ offset of the carrier frequency. This requirement is set for a static environment, meaning a propagation model with no multipath components or Doppler shift. This requirement results in a receiver that will filter out-of-channel interference, so low-power IEMI is only effective when it is in-channel.

A long list of countermeasures against jamming attacks are listed in [95]. A possible protection technique against jamming is the implementation of frequency hopping. By rapidly changing the carrier among many frequency channels the jammer is forced to spread its energy over a wide bandwidth, and thereby decreasing the power spectral density of the in-channel interference. The TETRA standard does not support frequency hopping, however, modern TETRA base station are equipped with jamming detection techniques. If an interferer is detected it has the possibility to switch to a different frequency channel. This is not a solution against a jammer with frequency agility, since it can also switch to the different channel. Another solution against jammers can be found in the application of smart antennas. Smart antennas can suppress the antenna gain in the direction of the jammer, and hereby increase the SIR at the output of the antenna. Most systems employing countermeasures, aim at decreasing the JSR by affecting the parameters shown in Eq. (3.4) in their advantage.

\subsection{Experimental testing method}

As mentioned earlier, the TETRA standard only describes the air interface, so the vulnerability to IEMI is different from manufacturer to manufacturer. For this reason experimental testing methods are necessary to give accurate and reliable results of the robustness of a base station against interference. In the next sections, we will present a method to measure the $P_{O B}$ over a wide frequency band, and a method to measure the raw BER of the uplink under different interference scenario's. The 


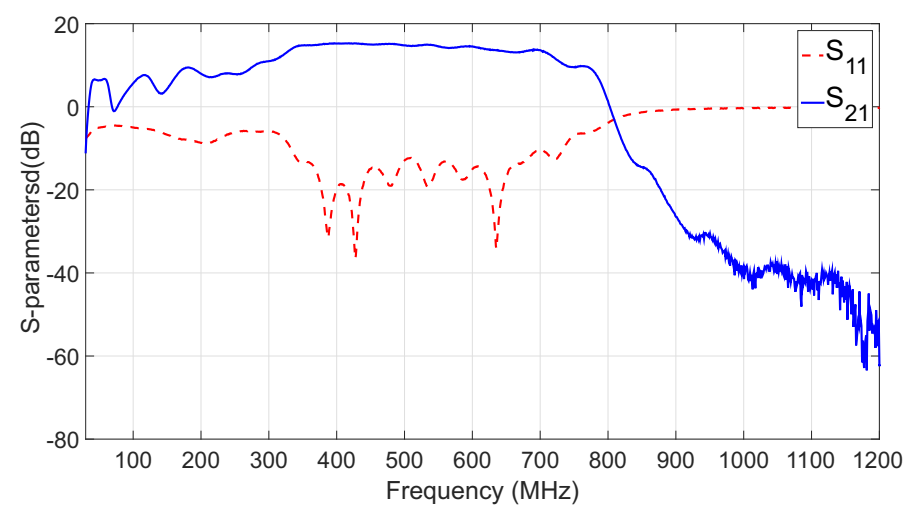

Figure 3.6: S-parameters of a LNB as a function of frequency.

measurements are conducting on a TETRA base station, as described in Section 3.3, but the methods are applicable to any wireless receiver.

\subsubsection{Gain compression}

At a single frequency point the gain curve of the LNB can be measured by directly feeding a signal to the input of the LNB, and connecting one of the output to a spectrum analyzer. Next, we increase the input power with small steps, while measuring the output power with a spectrum analyzer. If we plot the measured gain curve next to the extrapolated small signal gain curve we get a graph comparable to Fig. 3.5 and we can derive $P_{1-\mathrm{dB}}$.

The S-parameters from an LNB from input to one of the outputs has been measured with a network analyzer and the results are presented in Figure 3.6. As can be seen, the LNB has a gain over $10 \mathrm{~dB}$ from $280 \mathrm{MHz}$ to $740 \mathrm{MHz}$ meaning it can be used over a wide frequency range. The BPF determines the $5 \mathrm{MHz}$ frequency band where the TETRA base station receiver operates and different base stations can have different pass bands. This means that the susceptibility of the LNB needs to be analyzed over the complete frequency band where it can operate. The in-band gain, $380-400 \mathrm{MHz}$, is $15 \mathrm{~dB}$.

It is interesting to investigate the $P_{O B}$ over a wide frequency band, since the EMI can be both in-band and OOB. The investigated base station has a front end BPF that selects a $5 \mathrm{MHz}$ band and has a $80 \mathrm{~dB}$ attenuation for OOB interference, but this is not necessarily the case in other receivers. With the method we will present it is possible to measure $P_{O B}$ of an RF front end, without filter, over a wide frequency band. The results gives information which can set requirements on the front end filter. 


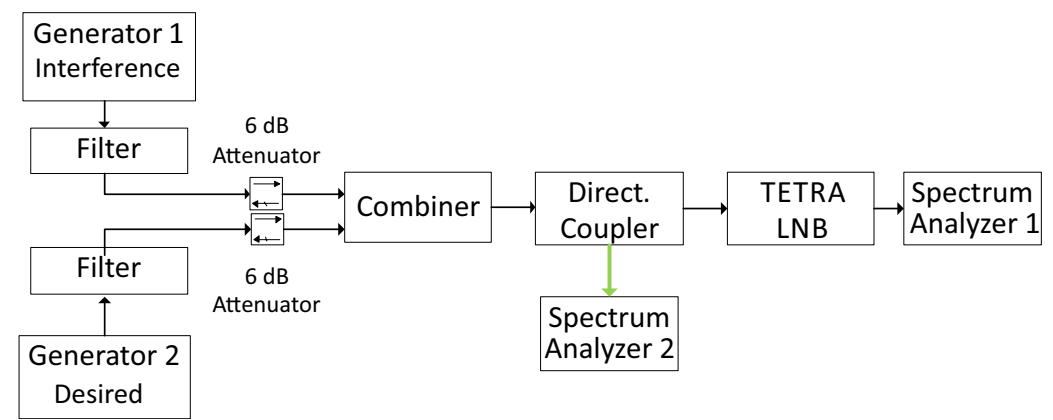

Figure 3.7: Measurement set-up for determining $P_{O B}$.

The method we use is based on the requirements for the control of EMI characteristics of electronic equipment described in MIL-STD-461E [99]. The basic concept is to apply out-of-band signals while monitoring the receiver for degradation, but in the standard only a very general test set-up is shown, and it is not specified how to quantify the degradation of the receiver. It is stated that the required test equipment, set-up, procedures, and data presentation should be determined on a case-by-case basis.

The schematic of the test set-up we used to measure $P_{O B}$ over a wide frequency band is given in Figure 3.7. It is a conducted susceptibility test to get accurate results. The description of the method is as follows: one signal generator, Generator 2, generates the in-band desired signal and the other generator, Generator 1, will generate the EMI. The desired signal should be well below $P_{1-d B}$ such that it does not contribute significantly to the saturation of the receiver.

To determine $P_{O B}$, firstly, Generator 2 is transmitting a small continuous wave (CW) desired signal at a fixed frequency, and the output power of the front end LNB is measured with a spectrum analyzer, SA 1. Next, Generator 1 will be switched on and starts transmitting a $\mathrm{CW}$, the blocker, at the frequency of interest. The power of the blocker is gradually increased until the output power of the desired signal at the LNB is decreased by $1 \mathrm{~dB}$, i.e. $1 \mathrm{~dB}$ compression, which is measured by SA 1. The $P_{O B}$ is determined by measuring the input power of the interfering signal with Spectrum Analyzer 2. The procedure is repeated for every frequency point of interest, and as a result, we get the $P_{O B}$ as a function of frequency. This set-up can also be used to monitor possible spurious emissions arising in the front end due to non-linearity.

It is important to verify that the signals at the input of the LNB are only the intended signals. For this reason, the set-up includes filters, 6-dB attenuators, and a directional coupler. The filters are connected, if necessary, to the output of the generators to filter the possible spurious harmonics generated in the signal generators. The 
generators are connected to 6-dB attenuators, which function as wideband isolators, to prevent unwanted reflection affecting the signals. Dedicated isolators are often not suited for this measurement, because they are narrowband and the measurements we perform can be extremely wideband.

A resistive splitter can be used as a wideband combiner. The resistive splitter we use has a loss of $6 \mathrm{~dB}$ from one port to another, whereas a 3-dB combiner only has 3 -dB loss. But again, a 3 - $\mathrm{dB}$ combiner is often not suited for this measurement, because they are narrowband and are therefore unable to combine two signals with a large frequency difference. Finally, a directional coupler is used to verify that the signals appearing at the input of the LNB are actually the intended signals, and to monitor the power of the interference.

\subsubsection{BER}

The schematic of the test set-up to measure the raw BER of the uplink of TETRA can be seen in Figure 3.8. Again, it is a fully conducted susceptibility test set-up. With this method we can measure the relation of the SIR at the input of the base station receiver and the BER.

A computer controlled BS will transmit a synchronization pattern to a Radio Communication Tester (RCT) that behaves as a TETRA MS. The RCT we use is the IFR 3901 Digital Radio Test Set. The RCT will synchronize to the BS and it will transmit a signal with a known bit pattern. The EMI is superimposed on the TETRA signal using a combiner. A directional coupler is used to monitor the power of the interference that is being received by the TETRA front end of the BS. The BS will analyze the BER and the results are displayed on the computer. This measurement is controlled by dedicated software installed on the computer.

In this setup, we can use narrowband dedicated isolators and a 3-dB combiner since the signals of interest will be in-band. The advantage of dedicated isolators is that the isolation greatly exceeds the $6 \mathrm{~dB}$ from the attenuators, meaning the $\mathrm{RCT}$ and interference generator are fully isolated.

The set-up shown in Figure 3.8 allows to investigate the impact of different interference scenarios on the quality of the wireless link. For example, the IEMI can be generated as $\mathrm{CW}$ interference, pulse interference, or as wideband noise interference (if within bandwidth of the isolator). Using $\mathrm{CW}$ interference, it is possible to analyze the selectivity of the superheterodyne receiver. Another possibility is to analyze the BER of the wireless link, while the front end is saturated, i.e. desensitized, by the interference. 


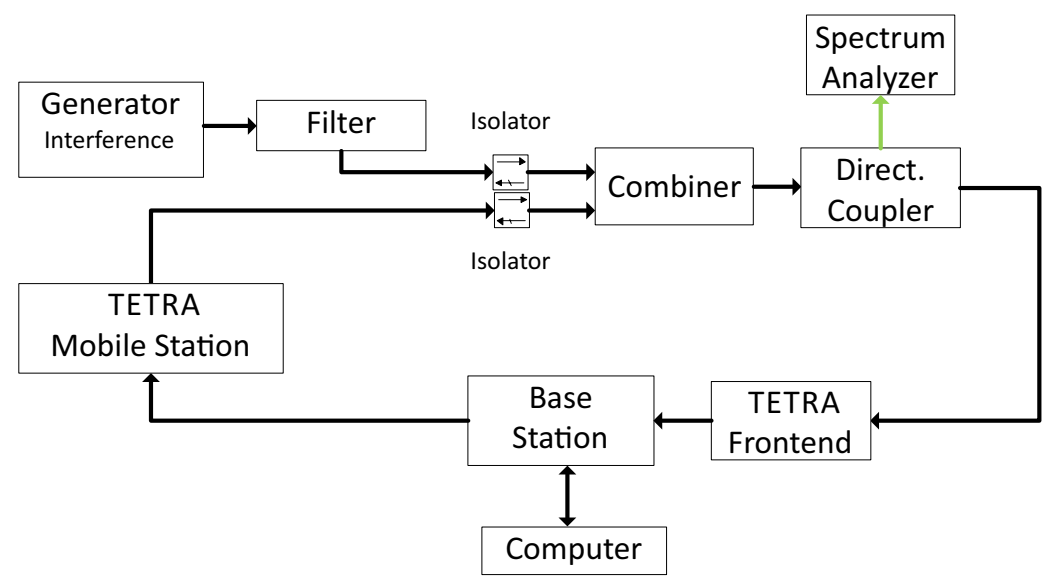

Figure 3.8: Test set-up to measure the BER of the uplink in the presence of an interferer

\subsection{Experimental results}

\subsubsection{Gain compression}

The gain curve of the LNB is measured at $388.5 \mathrm{MHz}$ and the input power from the signal generator is increased from $-40 \mathrm{dBm}$ to $20 \mathrm{dBm}$ with steps of $2 \mathrm{~dB}$. For every power step, the output power is monitored with a spectrum analyzer. The result is depicted in Figure 3.9. As can be seen, the output power flattens from approximately $-4 \mathrm{dBm}$ and for higher input power it can be seen that the receiver is operating in the saturation regime. The $1-\mathrm{dB}$ compression point was measured to be $-4 \mathrm{dBm}$.

Compression measurements, using the set-up as shown in Figure 3.7, were performed on an individual LNB, excluding a BPF, over a wide frequency band to analyze the compressive behaviour of the LNB for OOB EMI. The $P_{O B}$ was determined from $80 \mathrm{MHz}$ up to $900 \mathrm{MHz}$ with frequency steps of $10 \mathrm{MHz}$. The desired signal was set at $395 \mathrm{MHz}$ with a power of $-53 \mathrm{dBm}$ at the input of the TETRA LNB. The results are depicted in Figure 3.10. The lowest measured $P_{O B}$ is at $800 \mathrm{MHz}$ and is $-11 \mathrm{dBm}$. For frequencies exceeding $800 \mathrm{MHz}$ the compression points increased rapidly, because most power is reflected at the input port which can be seen in Figure 3.6.

\subsubsection{BER}

As mentioned before, the IFR 3901 functions as a TETRA mobile station. The losses from the RCT to the TETRA front end has been calibrated such that the output power selected at the IFR is the actual power at the input of the front end. The frequency of the TETRA signal is fixed at $387.5 \mathrm{MHz}$ and has a bandwidth of 
$25 \mathrm{kHz}$. The BER was continuously analyzed over a sample size of 170.000 bits of TETRA traffic channel with a user data rate of $7.2 \mathrm{kbit} / \mathrm{s}$. The analyzed BER is the raw BER without any error correction. The interference signal generator emits a $\mathrm{CW}$ interference exactly in-channel at a frequency of $387.5 \mathrm{MHz}$. The power of the $\mathrm{CW}$ interferer was increased with steps of $1 \mathrm{~dB}$ from $-90 \mathrm{dBm}$ up to power levels where the BER achieved its maximum.

In Figure 3.11, the BER curves are plotted for two TETRA signals with a power difference of $10 \mathrm{~dB}$ as a function of interference power. The absolute power levels of the TETRA signals is not presented, because of the sensitivity of these results, but the power of the TETRA signals are within the same range as the interferer. The results clearly show the non-linear relation between the SIR and the BER. These type of curves allow one to extract the in-channel CW EMI power that is necessary to achieve a certain BER for a constant TETRA signal. It should be clear that the power levels of the TETRA signal and the interferer are far below the compression point of the receiver. The increase in the BER is due to the in-channel interference masking the communication signal.

It can also be noticed that the two curves are similar, but shifted on the horizontal axis with $10 \mathrm{~dB}$, i.e., the power difference between the TETRA signals. This shows the constant SIR that is necessary to achieve a certain BER. Additional measurements were conducted to confirm these observations. Measurements showed that for a BER of $4 \%$ the SIR is constant, and independent on the absolute power levels. These measurements were performed over a power range of the TETRA signal from -95 $\mathrm{dBm}$ to $-50 \mathrm{dBm}$.

Finally, we measured the BER as a function of frequency of the interference to investigate the selectivity. First, the interference frequency was set exactly in-band

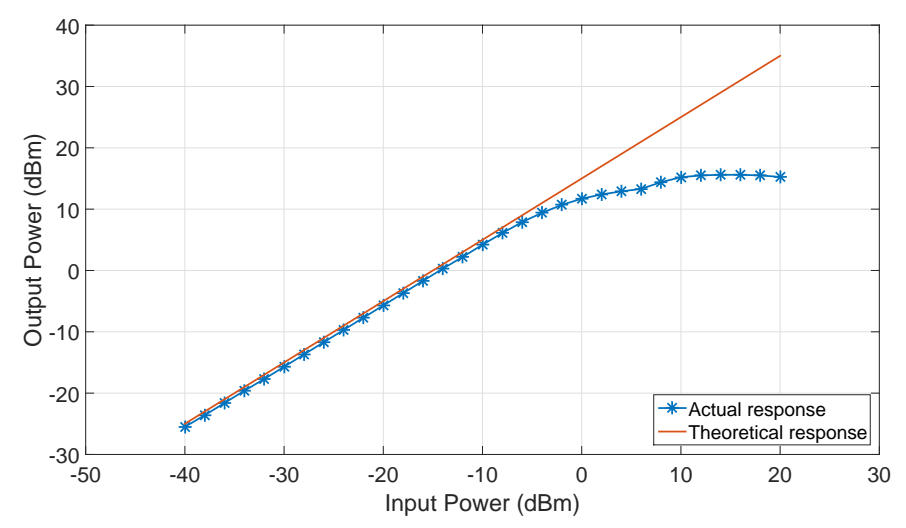

Figure 3.9: Gain curve of the LNB at $388.5 \mathrm{MHz}$. 


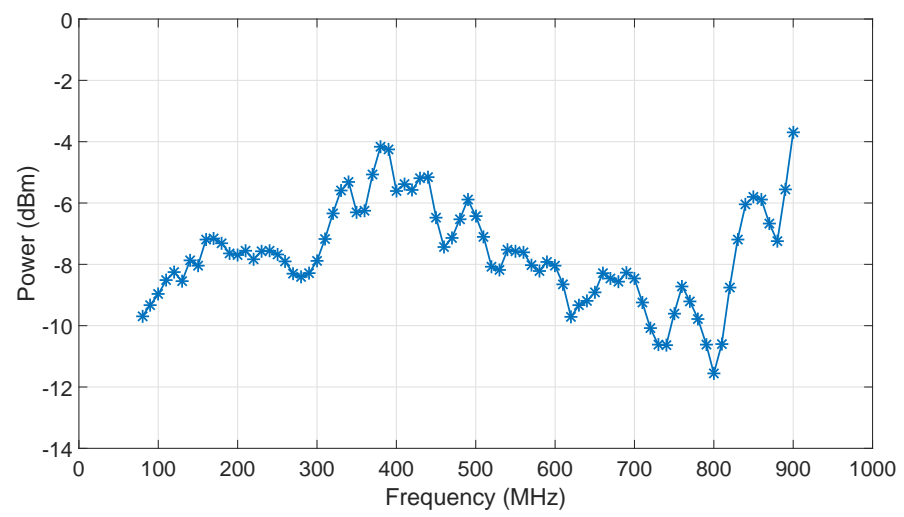

Figure 3.10: $P_{O B}$ of the TETRA LNB as function of frequency.

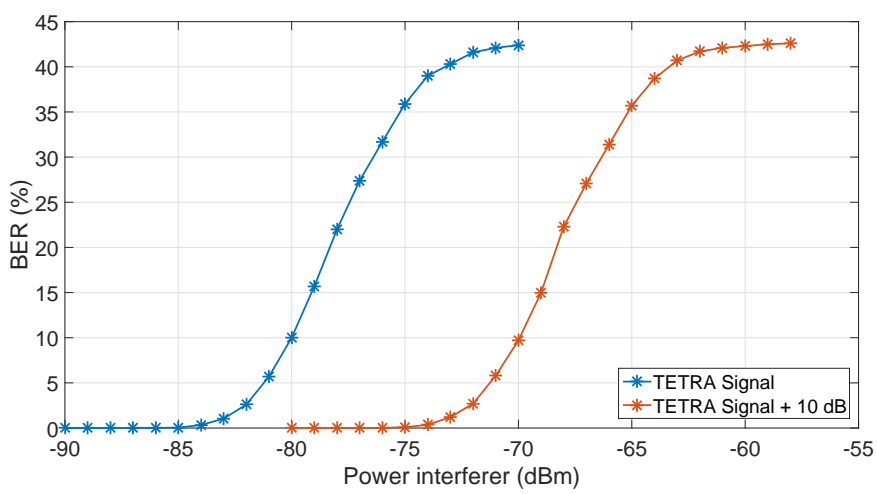

Figure 3.11: BER as a function of interference power for two TETRA signals of arbitrary power level. The power difference between the two TETRA signals is 10 $\mathrm{dB}$.

at $387.5 \mathrm{MHz}$ and the interference power was increased until a BER was achieved of $10 \%$. Next, we swept the interference frequency $15 \mathrm{kHz}$ with steps of $1 \mathrm{kHz}$. The BER as a function of frequency was recorded and the results in Figure 3.12 clearly shows the selectivity of the receiver. For interference frequency offsets of $\pm 10 \mathrm{kHz}$ from the TETRA center frequency the BER reduces to $0 \%$. The data of the TETRA signal is spread over a band of $18 \mathrm{kHz}$, whereas the channel width is $25 \mathrm{kHz}$.

The quality of the channel filtering in the base station is tested by monitoring the BER, while we increase the power of an in-band, but out-of-channel, interferer. The interference frequency was set at $1 \mathrm{MHz}$ of the TETRA center frequency (which is obviously out-of-channel), and the TETRA signal power is set at $-80 \mathrm{dBm}$. The power 


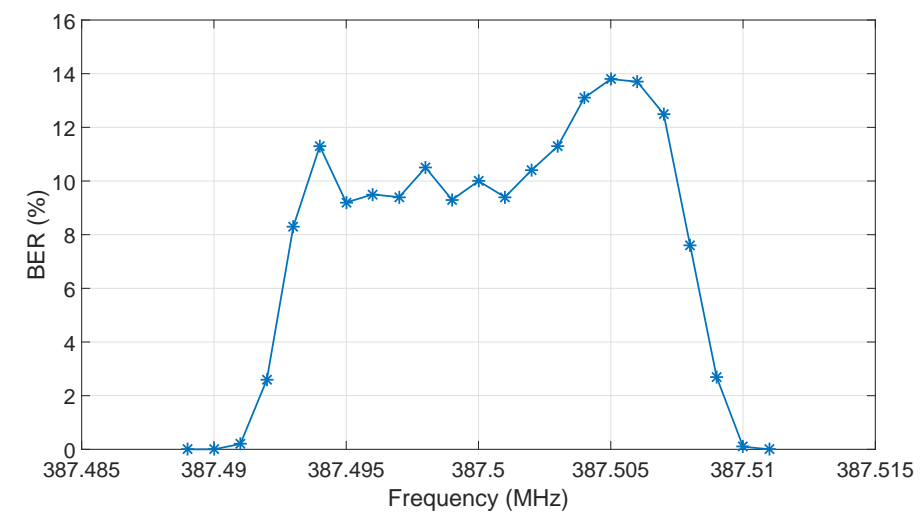

Figure 3.12: BER as a function of frequency of the interference. The power of the interference is set such that the BER is $10 \%$ (blue curve) at $387.5 \mathrm{MHz}$.

of the interference was increased up to $+10 \mathrm{dBm}$ and the BER was still $0 \%$. So even though the interfering signal is very strong, and completely saturating the receiver, see Fig. 3.9, the base station is still able to correctly detect the symbols of a $-80 \mathrm{dBm}$ TETRA signal. Of course, gain compression of the receiver leads to a reduced SNR and sensitivity. However, if the TETRA signal is well above the sensitivity levels, which is $-106 \mathrm{dBm}$ in a dynamic environment, it is possible to detect the symbol.

\subsection{Discussion}

The analysis on front door coupled IEMI presented in Section 3.4 is valid for any wireless receiver. The measurement set-up presented in MIL-STD-461E [99] has been further developed to specifically test front ends of wireless receiver to investigate the $P_{O B}$ over a wide frequency band. The experimental set-up and measurement methods presented in Section 3.5 are generic and can be used for any wireless receiver to determine its robustness against radiated IEMI.

The quantitative results presented in this chapter are only valid for the investigated base station, so it cannot be generalized. Base stations can be different from manufacturer to manufacturer as long as it applies to the minimum technical characteristics described in [80].

The base station is not protected against damage due to high power in-band EM interference, because there is no limiter mounted in front of the LNA. A comprehensive study conducted in [83-85] showed that the susceptibility level of a general LNA can be estimated at $34 \mathrm{dBm}$. Commercially available EM sources are able to exceed this power threshold at a large distance. A limiter mounted in front of the LNA would 
increase the robustness, and make the front end less susceptible for damage due to in-band IEMI. From a functional point of view, a limiter would introduce additional noise in the receiver chain, and this might be the reason that many base stations are not equipped with this component.

Interference in-band can saturate the front end which will lead to a decrease in the receivers sensitivity. Compression measurements have shown that in-band interference saturates the receiver at $-4 \mathrm{dBm}$. If the IEMI is out-of-channel, a saturated receiver can still correctly detect a TETRA signal as long as the power level is well above the sensitivity level. The sensitivity of the receiver does decrease with increasing gain compression. Measurements showed that the selectivity of the base station is high, i.e., the superheterodyne receiver filters out any out-of-channel interference in the receiver chain.

It is not possible to show a BER threshold for which the transmission of speech fails since TETRA is flexible in terms of different coding rates and error protection [75]. However, in [97] it is stated that the critical input error rates for most coding algorithms is below $25 \%$. From the results in Figure 3.11, it can be concluded that it is possible to jam TETRA signals with relatively low power signals. TETRA is designed for professional radio with an emphasis on security, but it is just as vulnerable to jamming attacks than any other civilian wireless system. In this chapter, only the impact of CW interference is investigated, but the set-up can also be used for many different type of interference scenarios, such as pulsed interference or wideband noise interference. For instance, it would be interesting to further investigate the impact of UWB pulses, as defined in [5], on a wireless receiver.

The difference between EMI and IEMI is that an adversary will always try to make use of the vulnerable frequency of the system. This means that the IEMI is expected to be in-channel, and so the communication will be disrupted. To overcome this weakness a complete different communication protocol at the physical layer would be necessary. Spread spectrum techniques such as frequency hopping are a mitigation technique against jamming, but it is not fully jamming proof. Wideband jammers are still capable of disrupting spread spectrum communication [97]. It is impossible to make a wireless system completely invulnerable against jamming attacks, but the goal of jamming countermeasures is to make a jamming attack cost more than the attacker's available resources [100].

It is easily concluded from the EMI analysis that the three interference mechanisms are fundamentally different in nature. The likelihood of occurence, as explained in Chapter 2.5.2, also differs per mechanism. Jamming has the highest likelihood of occurence, because of the availability and low-cost of RF jammers. More power is needed for damage and saturation of the receiver, and therefore the availability decreases.

To improve the robustness of wireless system against IEMI-by developing pro- 
tection techniques - it is necessary to have involved experts in various disciplines. Developing a robust communication system requires effort from software engineers, radio engineers, antenna engineers, microwave engineers, and EMC specialists.

\subsection{Summary and conclusions}

In this chapter, three different interference mechanisms are recognized, i.e. physical damage of the receiver, saturation of the receiver, and masking the communication signal, which needs to be addressed separately. The interference mechanisms are fundamentally different in nature and therefore a robust system should be designed by experts from various disciplines.

The research presented showed that the analyzed base station is robust against OOB interference due to the high quality band pass filter mounted before the front end. EM signals OOB are attenuated over $80 \mathrm{~dB}$. For OOB interference the power needs to be at least $-10 \mathrm{dBm}$ to saturate the $\mathrm{LNB}$ of the receiver. It is safe to conclude that OOB interference has no impact on TETRA via the front door. High power in-band interference can damage the receiver, because there is no diode limiter implemented. With moderate power levels, meaning power levels below $10 \mathrm{dBm}$, interference only has a significant effect if it is in-channel; out-of-channel interference does not harm the communication.

An experimental method for determining the saturation levels of a LNB over a wide frequency interval is presented in this chapter. This method to determine the OOB compression levels are useful, because the results enable to extract the minimum constraints necessary for a front end filter. Next, a test set-up is presented which can be used to analyze the impact of many different interference scenarios on the quality of the wireless link.

In Chapter 2 it is concluded that IEMI is a serious threat for wireless communication, and in this chapter the relevant interference mechanisms are identified. The next step is the deeper investigation of the impact these interference mechanisms have on wireless systems. This is the topic for the next three chapters. The vulnerability of TETRA against intelligent jamming techniques is analyzed in Chapter 4. Chapter 5 discusses the robustness of remote-keyless-entry (RKE) systems against jamming attacks. Finally, Chapter 6 presents a further investigation of the blocking mechanism of a front end. 


\section{Chapter 4}

\section{Intelligent jamming}

In this chapter, the results are presented of a study on the vulnerability of TETRA to intelligent jamming attacks. The results are published in IEEE Transactions on Electromagnetic Compatibility [101].

\subsection{Background of intelligent jamming attacks}

It is well known that wireless networks are vulnerable to jammers. Extensive research has been performed on this matter [92-97]. A brief overview of studies on jamming attacks will be given in this section.

There are various attack models that can be deployed by jammers to disrupt wireless communication, with various levels of sophistication. In [93] four different models are described. The effect of the jammers can have two results: 1) the sender does not transmit the communication signal because the medium is sensed to be busy, or 2) the reception of the receiver is interfered due to the jamming signal. The four basic models in [93] are:

- Constant jammer

- Deceptive jammer

- Random jammer

- Reactive jammer

Most jammers can be described either by one or a combination of these models.

A constant jammer continuously emits interference. The deceptive jammer is similar to this jammer, except the fact that a deceptive jammer continually injects regular information packets on the channel making it look like a regular transmission 
for observers. Both previously described models are power hungry. The random jammer is more power efficient and instead of continuously transmitting interference it alternates between sleeping and jamming. During the jamming stage it can either be a constant or deceptive jammer. The reactive jammer employs channel sensing. When the channel is idle it will not emit interference, but it starts transmitting when it senses activity on the communication channel.

In [97] various jamming techniques based on technical attributes are described. Based on the frequency and time attributes of the output signal the next classification can be applied to jamming:

- Noise jamming

- Tone jamming

- Swept jamming

- Pulse jamming

Noise jamming is generated by modulating a carrier with a noise signal. The bandwidth of the noise signal can be varied. It can be as wide as the entire frequency interval used by the victim system, or it can be much narrower, aiming at only a specific channel. Continuously jamming the complete frequency band is often referred to as barrage jamming. Tone jamming can consists of a single tone or more tone signals. Swept jamming is either a narrowband noise signal or a tone signal, which is swept all across the frequency interval of interest. Pulse jamming transmits the interference in a pulsed way.

As stated in Chapter 2, low-complexity RF jammers are easily acquired and very effective in disrupting wireless communication. However, these low-complexity jammers can be easily detected and are not very energy efficient. As an example, at the Nuclear Security Summit 2014 in the Hague, the Netherlands, the Dutch Radio Agency was continuously monitoring the RF spectrum. If an adversary would have tried to disrupt the communication of the security service using crude jamming devices, they would have been immediately detected, located and neutralized. This is the most effective countermeasure against crude jammers that emit interference across the entire frequency band of operation.

The detectability of a jammer, hence the possibility to undertake countermeasures, degrades the risk potential of an IEMI source. For this reason, intelligent jammers have been developed to increase jamming efficiency. The most commonly used criteria to determine jamming efficiency are: energy efficiency, probability of detection, level of DoS, and resistance to physical layer anti-jamming techniques [96]. All of these criteria are important, but depending on the situation one of these criteria will be more relevant. 
Intelligent jammers exploit weaknesses in higher layers of the Open Systems Interconnection (OSI) model to impair the correct functioning of the communication system. For instance, smart jammers will only target specific parts of the signal which are critical. Often an intelligent jammer targets only the control signals, which are periodically sent, and in this way paralyse the complete system. This implies that an intelligent jammer requires extensive information of the communication protocol of the victim system and sensing capabilities to be effective. This increase in complexity does decrease the availability of these type of sources, having a reducing impact on the risk potential.

Automated jamming detection systems are described in [93,95,102]. Detection is often based on a combination of metrics such as signal strength consistency checks, packets delivery ratio (PDR) and carrier sensing time. In normal operating conditions, a low PDR should be correlated to a low signal strength if it results from legitimate causes. For example, this situation can occur if the mobile station is too far away from the base station. However, if the received signal strength is high, yet the PDR is low, this is a strong indication that the wireless link is being jammed.

Countermeasures against regular jamming attacks, such as spread spectrum techniques and smart antennas, are obviously also increasing the robustness against intelligent jammers. Applicable measures against smart jammers are to scramble the control channels, or any other critical channel, in a pseudo random way [103]. Based on the scrambling scheme, a legitimate user would be able to receive the control channel, but a jammer would have to jam the complete signal to be effective. The goal of anti-jam methods is to force the adversary to deploy barrage jamming.

In the remainder of this chapter, the ability of TETRA to resist smart jamming attacks is investigated. By recognising the possible vulnerabilities it is possible to take adequate countermeasures and increase the security of the system.

\subsection{Vulnerabilities of TETRA protocol}

TETRA has been tested with Additive White Gaussian Noise, but smart jamming attacks focussing specifically on TETRA have not yet been investigated. The vulnerabilities of the TETRA protocols are discussed in this section.

\subsubsection{Interfering with the voice data}

Jamming of the sent voice data is the most easy and crude way to deny users from service. However, it requires a lot of energy since it is required to interfere with the voice data continuously. The speech data passes error control schemes and the data is split into bits of different priorities. The most important bits receive a lot of error protection and therefore interfering with these data bits requires corrupting 
the complete bit stream during the conversation. Since the jammer needs to transmit interference signals continuously, it can be detected relatively easily by measuring the received signal strength [102]. If the interference is detected, the system can take countermeasures to reduce the impact of the jammer.

\subsubsection{Distributed Denial of Service (DDoS) attacks}

In TETRA, the upper Medium Access Control (MAC) layer provides air interface encryption [77]. It is therefore difficult to obtain the original messages. Furthermore, it is difficult to spoof the communication system since all TETRA devices have TETRA Equipment Identification (TEI) numbers, which uniquely define each device [75]. Without a registered TEI number it is not possible to start a conversation. All registered numbers are stored in databases and once a device is obsolete or is lost, the number can be stripped of its permissions to make calls and send data.

A commonly used attack is a DDoS attack [104]. There are several ways to perform such an attack, but the main goal is always to deny users from service. For example, a DDoS attack can create a large number of communication requests that saturates the target device, so that it cannot respond to legitimate traffic. These attacks can also be generated against TETRA and do not necessarily require valid TEI numbers. However, it requires more power to generate the many synchronisation messages at the base station compared to generating an interference signal at the right time. Also, most importantly, the jammer can be more easily detected since the target device receives many messages with an invalid TEI number.

\subsubsection{Interfering with the TDMA synchronisation}

There is a more intelligent way to interfere with the system than just simply occupying the channel by transmitting continuously. The system is vulnerable to interruptions of the correct control messages. The advantage of this technique compared to the naive jammers is that the jammer is more likely to stay covert, since it does not have to send signals continuously as in the case of the constant and deceptive jammer. It hits the critical control packets instead of sending random bits and corrupting random packets. Furthermore, the jammer is more energy efficient, since it is not constantly sending interference signals [102]. Interference signals acting on specific control data packets of TETRA have not been reported to the knowledge of this author.

TETRA uses TDMA and therefore the mobile and the base station have to synchronise each time a communication session is started. This synchronisation is not protected. The base station sends the unencrypted synchronisation block periodically. These blocks are known so that the mobiles can lock onto it [77]. If the synchronisation is disturbed, the mobile cannot synchronise with the base station and the 
communication link cannot be set up. This way of jamming requires listening to the channel and determining when the synchronisation block is sent and subsequently interfere with this signal. Jamming this signal will only work if the mobile has not established a connection with the network already. However, since the mobile sets this connection at start, a non-critical moment, it is not very effective.

\subsubsection{Interfering with the Access Assignment Channel}

Another better possibility to paralyse the TETRA system is to interfere with the random access protocol. This protocol is based on slotted ALOHA procedures [105]. The slotted ALOHA procedures are extended with an access framing structure. The random access protocol with slotted ALOHA is used when a mobile wants to transmit an unsolicited message to the base station. The mobile station does not have a reserved channel and has to use this protocol.

The base station sends so called "Access Codes". There is a maximum of four possible access codes. The base station sends these codes to mark opportunities for the mobile stations to start a transmission. Mobile stations will only try to send traffic in these designated time frames. This way the control of collisions between access requests from different mobile stations is taken care off. It is also possible to provide different kinds of grades of service.

For convenience the TETRA frame structure is shown in Figure 4.1. One TDMA slot is 510 bits long. Each TDMA slot assigned for data transmission from base station to mobile station, i.e. a downlink, contains a Broadcast Block. The access codes are sent on the Access Assignment CHannel (AACH). The AACH is sent in the Broadcast Block of every downlink slot and it consists of 14 bits. Before these 14 bits are sent to the physical layer for transmission they are first encoded with a shortened Reed Muller code into 30 bits and then the resulting 30 bit long stream is scrambled. The mobile will wait for the correct access code before transmitting.

In the ETSI TETRA protocol standard the following is stated: "If the AACH is not decodable then both the corresponding uplink subslots shall be regarded as reserved" [77]. Thus it regards the uplink slot as not available for random access. So according to the protocol it is possible that the mobile will wait indefinitely if it cannot decode the AACH message. From the mobile station point of view it appears that the network is congested, since the devices cannot make new connections, but running conversations are not affected. However, if the base station contacts the mobile then it still can setup a link since it will reserve slots for the mobile to send its data. Nevertheless, the impossibility for the mobile station to setup a link impedes the system significantly. 


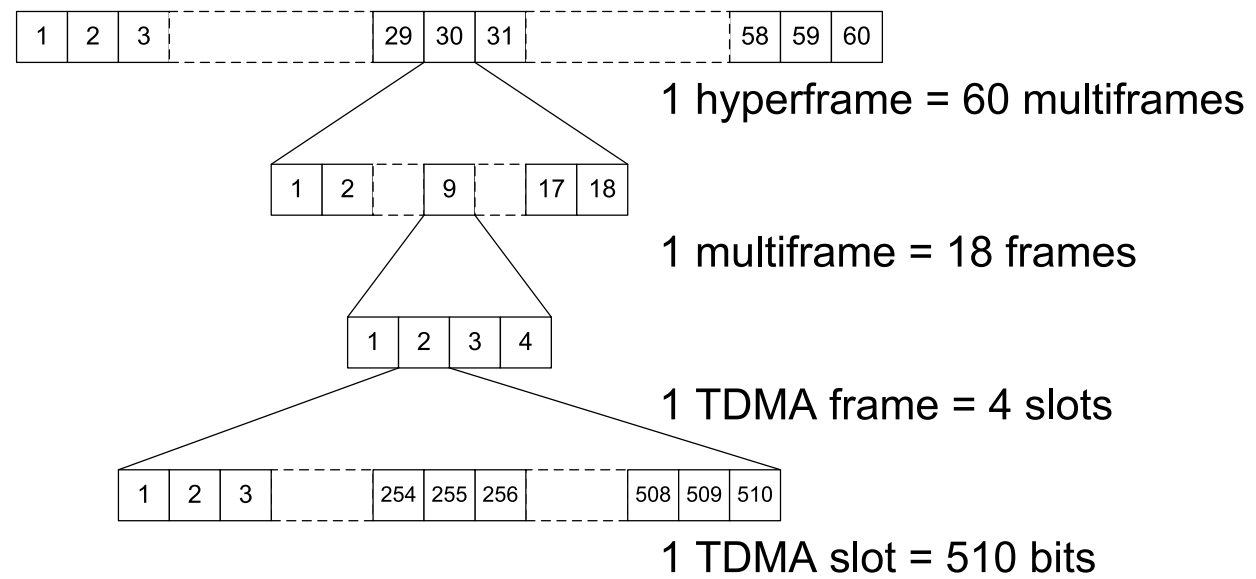

Figure 4.1: TETRA frame structure. Each TDMA slot assigned for downlink channels contains a Broadcast Block [77].

\subsection{Symbol errors on the physical layer due to interference signals}

To cause a denial of service, the physical signs of the control messages have to be corrupted. TETRA uses $\pi / 4$-DQPSK and the newer versions of TETRA use $\pi / 8$ DQPSK and quadrature amplitude modulation (QAM) modulation. This research focuses on the widely implemented $\pi / 4$-DQPSK modulation. This modulation scheme consists of two signal constellations as shown in Figure 4.2 and the modulation scheme switches between these two constellations for every consecutive symbol. In the left constellation, the points lie on $\pi / 4,3 \pi / 4,-\pi / 4$ and $-3 \pi / 4$. In the right constellation, the points lie on $0, \pi / 2,-\pi$ and $-\pi / 2$. The phase transitions between symbols for this modulation scheme are $\pi / 4,3 \pi / 4,-\pi / 4$ and $-3 \pi / 4$. Disrupting the signal on the physical layer is the obvious choice, since the shared nature of the medium makes it easy to access for interference signals.

To achieve errors in the modulation scheme the error vector magnitude (EVM) has to be increased to shift the constellation points over the decision boundaries of both constellations. The EVM is a measure of how much the constellation point is shifted away from the correct position in the constellation diagram.

An asynchronous continuous wave is one of the simplest signal to create an EVM that shifts the constellation points over the decision boundaries. The data signal superimposed by a continuous wave interference can be described as:

$$
\begin{aligned}
x_{n}(t)+m(t)= & a \cos \left(\omega t+\phi_{n}\right)+ \\
& b \cos \left(\omega t+\Delta \omega t+\phi_{m}\right)
\end{aligned}
$$




\section{Quadrature}

\section{Quadrature}
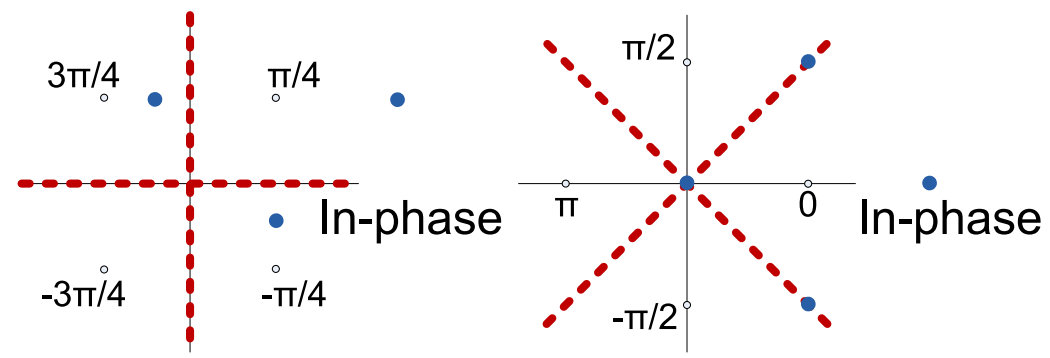

Figure 4.2: The constellation diagram of $\pi / 4$-DQPSK consists of a set of two signal constellations: QPSK and a $\pi / 4$ rotated QPSK. The small dots are the constellation points, the dashed red lines indicate the decision boundaries and the big dots are the constellation points when the $\pi / 4$-DQPSK scheme is interfered with a QPSK signal.

where $x_{n}(t)$ is the data signal, $m(t)$ the interference signal, $a$ the amplitude of the modulated signal, $\omega$ the carrier angular frequency, $t$ the time, $\phi_{n}$ the modulated phase, $\phi_{m}$ the phase of the interference signal, and $\Delta \omega$ the difference angular frequency between the modulated signal and the interference signal. The quadrature components of this combined signal can be described as:

$$
\begin{aligned}
I(t) & =a \cos \left(\phi_{n}\right)+b \cos \left(\Delta \omega t+\phi_{m}\right) \\
Q(t) & =a \sin \left(\phi_{n}\right)+b \sin \left(\Delta \omega t+\phi_{m}\right)
\end{aligned}
$$

where $I(t)$ is the in-phase component and $Q(t)$ is the quadrature component. The first terms in $I(t)$ and $Q(t)$ are the desired quadrature components of the data signal, and the second terms results from the asynchronous continuous wave interference signal. As a result, the received point in the constellation diagram after demodulation will lie on a circle around the ideal constellation point.

In a similar way a QPSK modulated interference signal is superposed on the $\pi / 4$-DQPSK. In this analysis the QPSK interference signal is synchronised with the $\pi / 4$-DQPSK signal in order to be able to push the points over the decision boundaries as shown in Figure 4.2. It is assumed that the signals are exactly synchronised to achieve the clear superposition of the $\pi / 4$-DQPSK and the QPSK. In a practical situation this is never the case. The phase noise and the frequency difference between the interference signal and the modulation signal will rotate the QPSK signal around the $\pi / 4$-DQPSK points. In addition, the sampling points to determine the symbols for the $\pi / 4$-DQPSK and the QPSK are not in synchronisation. Therefore the QPSK signal will not be sampled on the four points, but also somewhere along the signal trajectory between the four points. 


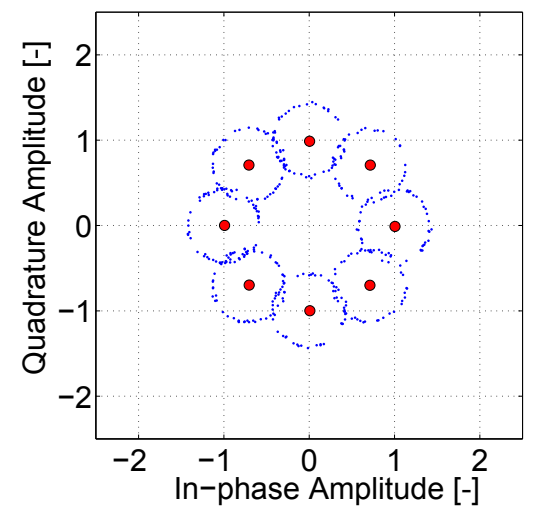

(a)

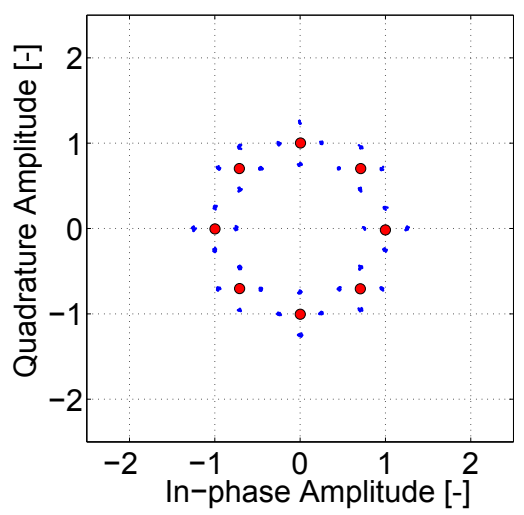

(b)

Figure 4.3: Constellation diagram of $\pi / 4$-DQPSK with (a) continuous wave interference signal and (b) synchronised QPSK interference signal. The red dots are the ideal constellation points, and the blue dots are the received points.

A standard Simulink model has been adapted to determine the effects of interference signals added to the channel [106]. If a continuous wave is added to the channel to interfere with the modulated signal it is expected that the interference signal is superimposed on the original constellation diagram. A QPSK modulated signal is also simulated as an interference signal. Figure 4.3a and Figure 4.3b confirm the superposition of the interference signals as expected by equation (4.2). The continuous wave interference signal caused an EVM that rotates around the ideal constellation points and the synchronous QPSK modulated interference signal caused an EVM consisting four points around the ideal constellation points.

The graphs in Figures $4.4 \mathrm{a}$ and $4.4 \mathrm{~b}$ show the spectra of the data signal superimposed by the interference signal. It can be clearly seen that the continuous wave creates a spike in the spectrum, while the power of the QPSK interference signal is divided over the whole spectrum.

\subsection{Intelligent TETRA jammer}

A possible threat for a TETRA system would be an intelligent jammer that interferes with the bits involved in AACH control. Jamming these control messages will deny users from service.

In the previous sections it is shown that the TETRA communication system can be disrupted, so the mobile stations cannot start calls. This is achieved by interfering with the $\mathrm{AACH}$ messages sent by the base station, which improves the jamming 


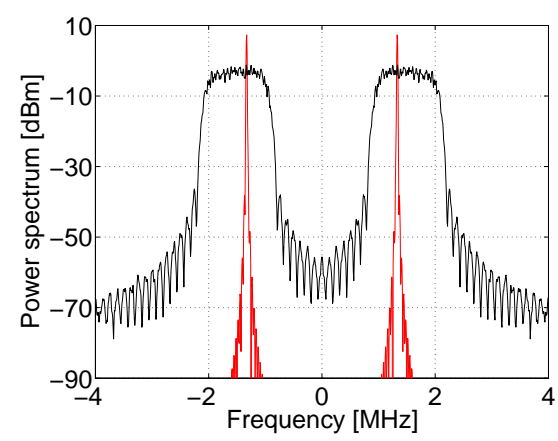

(a)

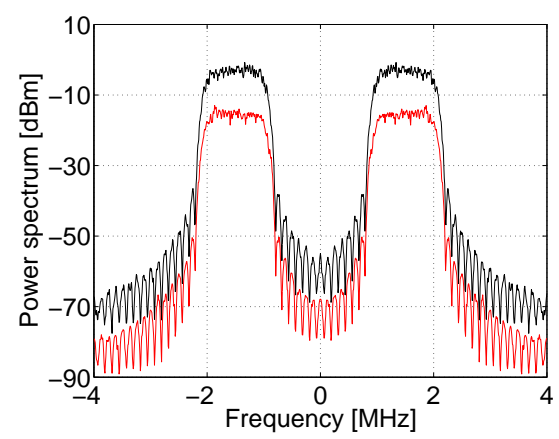

(b)

Figure 4.4: Spectrum of $\pi / 4$-DQPSK (black) with (a) CW interference and (b) QPSK signal interference.

efficiency considerably. The probability of detection of the jammer can be reduced by using a QPSK modulated signal as the interference signal, since the signal is divided over the spectrum as shown in Figure 4.4b. The discrimination between legitimate and adversarial traffic is the main challenge for detection of jammers [102]. In this case, it is not straightforward to discriminate jamming from the legitimate traffic scenarios using only the signal strength [102].

Other data besides the signal strength are analysed such as the PDR. Even if a network is congested the PDR always maintains a certain value, while an effective jammer decreases the PDR to a value close to zero [102]. However, this method cannot be applied to detect the jamming attack described in this paper. Conversations cannot be started by the mobile stations by attacking the AACH. There is no steep drop in PDR, since an ongoing conversation is not terminated.

As described in Section 4.1 advanced jamming detection strategies include combining the PDR and the signal strength. An intelligent jammer interfering with the $\mathrm{AACH}$ control circumvents this detection, since there is no data for the PDR, because no new conversation can be started and ongoing conversations are not interrupted.

So a jammer that interferes with the AACH and uses a QPSK signal is effective at disrupting the TETRA communication system, while being able to stay covert.

\subsection{Experimental results}

In Section 4.3, it is shown that an interference signal that is distributed over the spectrum is more likely to stay covert than a continuous wave. In this section the concealment of the interference signal and the superposition of the interference signal with the modulated signal are verified. 


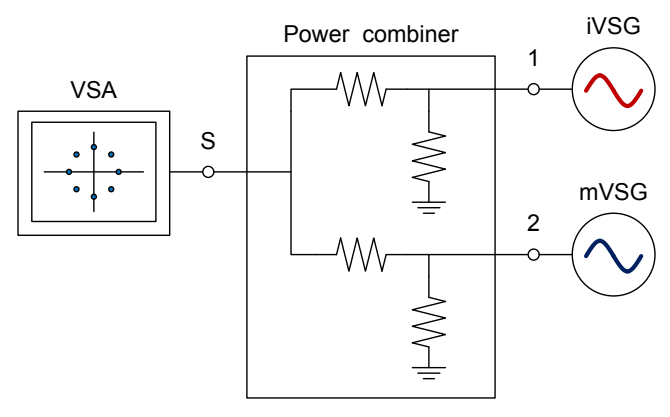

Figure 4.5: Measurement setup modulation scheme superposition; a modulating vector signal generator (mVSG), an interfering vector signal generators (iVSG) connected via a power combiner to a vector signal analyser (VSA).

A vector signal analyser (VSA) was connected with two vector signal generators (VSG) via a power combiner. The measurement setup shown in Figure 4.5 was used to measure the superposition and spectra. The VSA was an Agilent PXA Signal Analyzer N9030A, the modulating vector signal generator ( $\mathrm{mVSG}$ ) to create the modulation signal on a frequency of $390 \mathrm{MHz}$ was an Agilent E4438C ESG VSG, and the interfering vector signal generator (iVSG) to create the interference was an Agilent E8267D PSG VSG. The used power combiner was an ZFRSC-123-S+ from Mini-Circuits. The VSA measured the constellation diagram and the spectrum. The following settings were set for the mVSG: centre frequency at $390 \mathrm{MHz}$, power at -40 $\mathrm{dBm}, \pi / 4$-DQPSK modulation, and a symbol rate of $18 \mathrm{kbps}$. The iVSG used two different interference signals: a continuous wave and a QPSK modulation scheme. The power of the interference was set at $-50 \mathrm{dBm}$ at the centre frequency and at $1000 \mathrm{~Hz}$ above the centre frequency of the modulated signal. The iVSG and mVSG were synchronised and unsynchronised by connecting and not connecting the $10 \mathrm{MHz}$ reference signal, respectively. The iVSG sent random bits with QPSK modulation.

The constellation diagrams are shown in Figures $4.6 \mathrm{a}$ and $4.6 \mathrm{~b}$ and the spectra are shown in Figures $4.7 \mathrm{a}$ and $4.7 \mathrm{~b}$. When the continuous wave had the same centre frequency and was synchronised via the $10 \mathrm{MHz}$ reference signal with the $\pi / 4$-DQPSK signal, the interference did not affect the constellation diagram. The continuous wave adds a constant shift in the diagram, but the VSA compensates for this constant shift. Therefore, this shift is not visible in Figure 4.6a. The QPSK signal, however, does create an error and as expected the QPSK signal is superimposed on the $\pi / 4$-DQPSK signal. Measurements were performed with larger amplitudes causing the VSA to lose lock on the $\pi / 4$-DQPSK signal and the constellation could not be reconstructed. The EVM readings also increased and varied greatly.

When the continuous wave was unsynchronised and set at $1000 \mathrm{~Hz}$ above the 


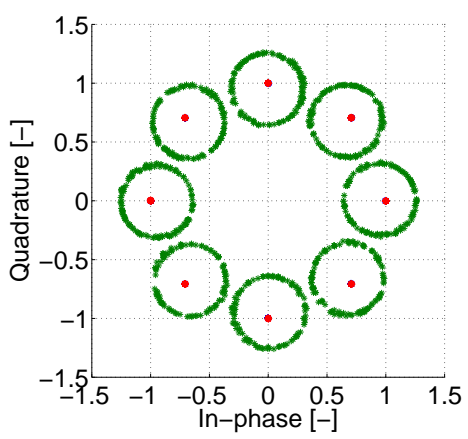

(a)

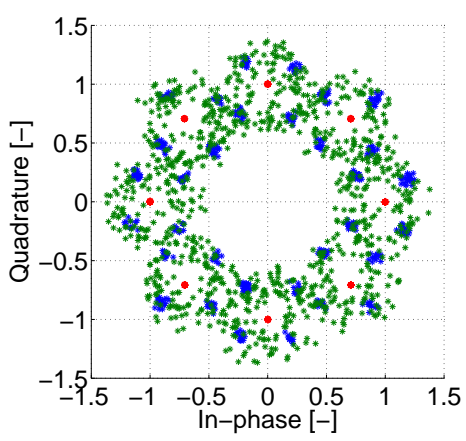

(b)

Figure 4.6: Constellation diagram of $\pi / 4$-DQPSK (red pluses) with (a) asynchronous continuous wave IEMI at $1000 \mathrm{~Hz}$ above the center frequency (green asterisks), and (b) synchronized QPSK-modulated IEMI signal (blue dots) and asynchronous QPSKmodulated IEMI signal (green asterisks).

centre frequency, the constellation rotated, which is in accordance with equation Eq. (4.2).The asynchrony and setting the frequency $1000 \mathrm{~Hz}$ above the centre frequency of the $\pi / 4$-DQPSK resulted in a cloud of points around the constellation points when a QPSK interference was added. This is because the signal is on a trajectory to the QPSK points, but is not sampled at times when the QPSK points are reached. The phase noise also caused the constellation points to arc. Exact synchronisation with the TETRA signal is in practice hard to achieve and therefore the asynchronous results are more realistic.

The continuous wave created a larger EVM than the QPSK signal, however, in Figure 4.7a, two clear peaks can be seen at the centre frequency and $1000 \mathrm{~Hz}$ above the centre frequency, which are caused by the continuous wave interference. The QPSK interference does not cause a noticeably different spectrum to that shown in Figure 4.7b. The continuous wave at $1000 \mathrm{~Hz}$ above the centre frequency caused a larger EVM than the QPSK signal, while also causing a peak in the received spectrum.

The QPSK signal, however, did not create a noticeable difference in the received spectrum. The QPSK interference is more difficult to detect than a continuous wave, which is in accordance with the study performed by Mlezcko et al [107], where they show that a signal occupying a significant amount of the bandwidth requires a lower signal level. If the interference signal stays covert, then it is less likely that countermeasures against the interference signal will be taken. 


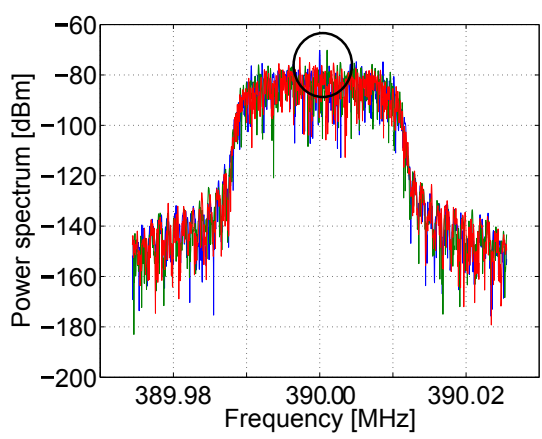

(a)

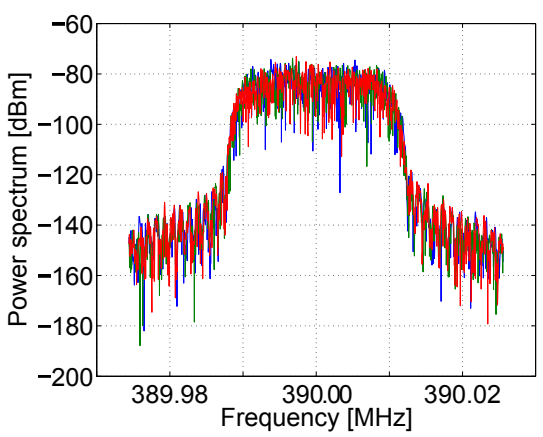

(b)

Figure 4.7: Spectrum of received signal at the VSA with (a) synchronized continuous wave IEMI signal on the center frequency (blue) and asynchronous wave continuous wave IEMI at $1000 \mathrm{~Hz}$ above the center frequency (green) and (b) synchronized QPSK-modulated IEMI signal on the center frequency (blue) and asynchronous QPSK-modulated IEMI $1000 \mathrm{~Hz}$ above the center frequency (green).

\subsection{Summary and conclusions}

An overview of general jamming attacks is presented in this chapter. This overview is based on relevant literature with respect to jamming attacks against wireless networks. The goal of countermeasures against jamming attacks - such as the scrambling of the control channels - is to force the adversary to deploy barrage jamming, which is not power effective and easily detected. Next, the protocol of TETRA was investigated to identify possible weaknesses.

From the analysis, we can conclude that TETRA can be disrupted by an intelligent jammer. The slotted ALOHA protocol can be interfered by corrupting each access assignment channel block, since the TETRA protocol states that the mobile station will wait indefinitely before transmitting until the access assignment channel can be decoded. This jamming attack is only effective if the victim mobile device wants to set-up a connection. If the base station contacts the mobile then it still can set-up a link since it will reserve slots for the mobile to send its data.

The study also showed that an intentional electromagnetic interference $10 \mathrm{dBm}$ lower than the intended signal was able to create a large EVM. The continuous wave interference caused a larger EVM than the QPSK modulated interference. However, a QPSK modulated interference stays covert, while a continuous wave with the same power causes a noticeable peak in the received spectrum compared to the situation without any interference. Therefore, the TETRA protocol is vulnerable to a QPSK modulated interference signal corrupting the $\mathrm{AACH}$, since it is hard to detect the 
interference signal.

After discussing intelligent jamming attacks against TETRA in this chapter, the next chapter will discuss the effect of a jamming attack on RKE systems. Although RKE systems are not directly part of a critical infrastructure, it does rely on wireless systems, and as such is an interesting case study to study the effects of a jamming attack and to identify possible improvements. 


\section{Chapter 5}

\section{Jamming attacks against remote keyless-entry systems}

In this chapter, the results are presented of a study on the vulnerability of remote keyless-entry (RKE) systems against jamming attacks. The results are published in IEEE Transactions on Electromagnetic Compatibility [108] and presented at the IEEE International Symposium on Electromagnetic Compatibility in 2014 and 2015 $[109,110]$.

\subsection{Background on RKE systems and IEMI}

The remote keyless-entry (RKE) system is an electronic lock that controls access to vehicles or buildings by use of a wireless key fob carried by the user. The key fob is an electronic remote control that sends a unique code to the receiver, located in the car or the building, which communicates with the central locking system to control access. The advantage of an RKE system - as compared to traditional access systems with a mechanical key - is the higher level of flexibility and comfort in getting access.

The wireless communication of RKE in Europe operates in the license-free 433 $\mathrm{MHz}$ ISM band (433.05 MHz - 434.79 MHz). Most of the RKE systems employ oneway communication, using the key fob as RF transmitter. The key fob transmits bursts of data on a digital-modulated carrier. In most parts of the world, the used modulation is on-off keying (OOK), which is the most elementary form of amplitudeshift keying (ASK) [111].

A serious issue that arose with RKE is the security of the system. The open nature of the wireless medium makes it possible for intruders to gain information from the system and acquire illegal access. As an result, manufacturers have increas- 


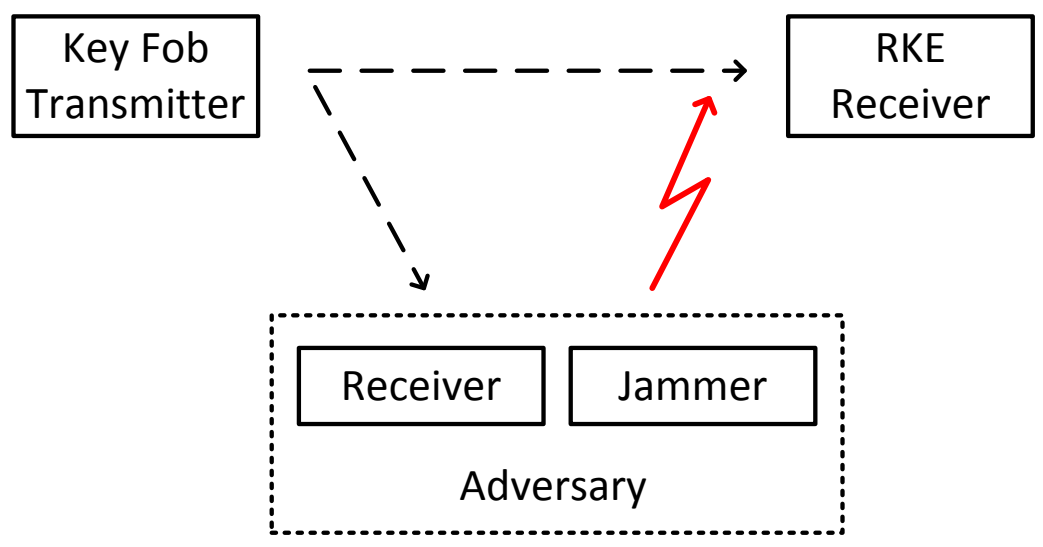

Figure 5.1: The scenario of jamming the signal from the key fob to the RKE receiver, while the adversary simultaneously receives the transmitted signal.

ingly secured the system by use of encryption and code algorithms on higher open systems interconnection (OSI) layers. For example, nowadays many RKE systems use the rolling code technique to prevent replay attacks [112,113]. In [113], an detailed analysis is made of possible attacks against the security of RKE systems.

More recent at DEF CON 2015 - one of the world's largers hacker conventions - a technique was presented that allows an intruder to hack RKE systems and acquire access to cars and garages [114]. At the heart of this technique is the ability of the intruder to jam the signal from the key fob to the RKE receiver, while at the same time the receiver of the intruder is able to receive and store the keyless-entry codes transmitted by the key fob. This is shown in Fig. 5.1. This technique, employing jamming on the physical layer, renders the coding algorithms such as rolling codes, on higher OSI layers to be useless. An detailed discussion of this techniques is presented in [115]. The threat of an adversary's ability to both jam an RKE receiver and receive the transmitted signal at the same time is the main interest of this paper.

From the aforementioned technique, it should be clear that the security of the RKE system can be improved by increasing the robustness of the receiver against jamming attacks. If the intruder is not able to jam the RKE receiver without jamming its own receiver, the technique described in [114] and [115] would not work. However, as concluded in Chapter 3, wireless communication is easily disturbed by IEMI. In this chapter, the weaknesses of RKE systems are investigated and suggestions are made for robuster systems against jamming attacks.

In Section 5.2, it will be shown that a poor selectivity makes a receiver extremely vulnerable against the jamming technique described in [114]. Two low-cost RKE systems for automotive applications are investigated in this section. After Section 5.2, the focus will be on the vulnerability of a superheterodyne receiver, commonly used 


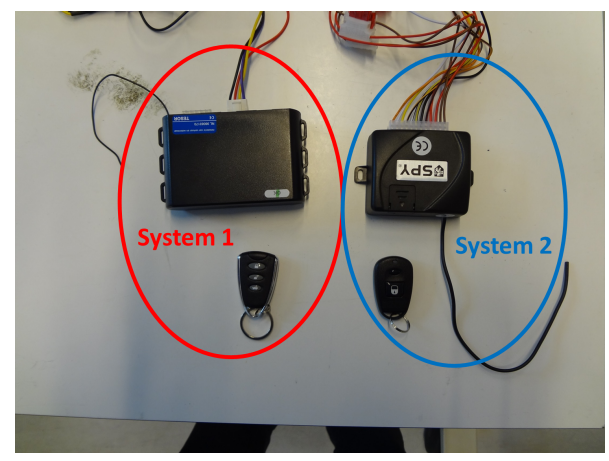

(a)

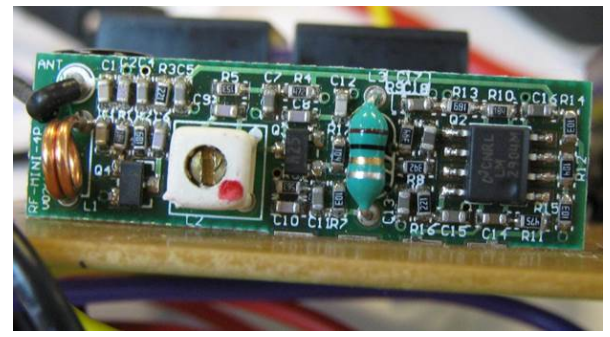

(b)

Figure 5.2: In (a) the two RKE systems bought online and in (b) a picture of the receiver circuit of system 1 .

in RKE applications, against pulsed interference. In Section 5.3, it is shown in what way pulsed interference differs from continuous interference and the reason why it can be more detrimental. This analysis is completed with simulations. In Section 5.4, an experimental study is presented to test the RKE heterodyne receiver. BER curves are shown of the receiver under various interference scenarios, including the effect of a varying pulse repetition frequency (PRF). Next, in Section 5.5, an improved receiver design that increases the robustness to pulsed interference is presented. Finally, in Section 5.6, conclusions are drawn with respect to the vulnerability of RKE systems against IEMI.

\subsection{Analysis of purchased low-cost RKE systems}

\subsubsection{Super regenerative receivers}

Most receivers in RKE applications are superheterodyne receivers. However, after purchasing two RKE systems for automotive applications, it was found that the receiver in these particular two systems are super regenerative receivers (SRR). This receiver technology is simple and low-cost, since it consists of relatively few electronic components. In RKE applications SRR are used, because they are very low cost, consume little power, and are well-suited for OOK detection [116]. The purchased RKE systems (system 1 and system 2) are depicted in Figure 5.2a and the receiver circuit of system 1 is depicted in Figure 5.2b.

The details of SRR are explained in $[117,118]$. The most important drawback of SRRs is their poor selectivity. This results in a high susceptibility for interference from an adjacent channel. Especially in an license-free ISM band this can result in 


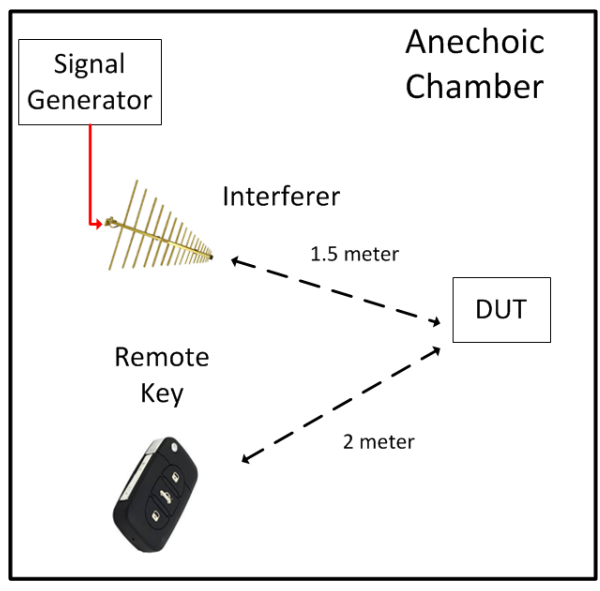

(a)

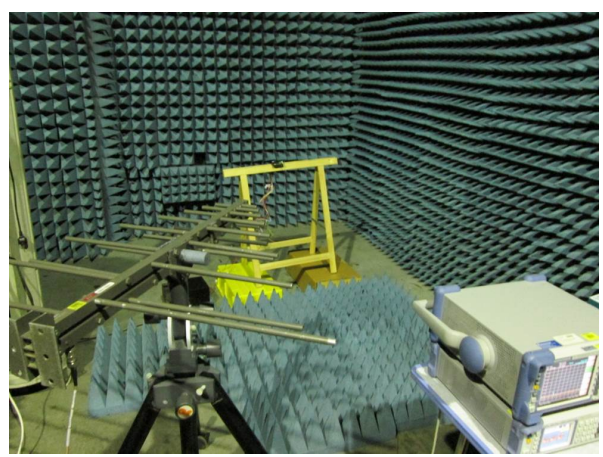

(b)

Figure 5.3: Measurement method with (a) schematic of the test set-up in the anechoic chamber and (b) a picture of the actual set-up.

many issues.

In this section, the focus is on the selectivity of the two purchased RKE systems which employ SRR. However, it has to be clear that this is a low-end system, and many RKE systems are equipped with superheterodyne receivers. The vulnerability of these systems is later investigated.

\subsubsection{Selectivity of purchased systems}

The selectivity of the RKE systems and the robustness against interference was tested in an anechoic chamber. The test setup is depicted in Figure 5.3a. The receiving part of the RKE system was illuminated by a log-periodic antenna at a distance of 1.5 $\mathrm{m}$. The antenna was connected to a signal generator to emit the continuous wave interference. The interference was being swept from $420 \mathrm{MHz}$ up to $460 \mathrm{MHz}$ with steps of $500 \mathrm{kHz}$. This frequency interval was chosen, because the receiving antenna is tuned to this frequency range by the system designer. From $433 \mathrm{MHz}$ to $436 \mathrm{MHz}$ steps of $200 \mathrm{kHz}$ were taken.

The key fob was fixed at a distance of $2 \mathrm{~m}$ from the receiver. The electric field (E-field) generated by the key fob was measured using the log-periodic antenna with a known antenna factor. The remote control of system 1 generates an E-field of 85 $\mathrm{dB} \mu \mathrm{V} / \mathrm{m}$ at a distance of $2 \mathrm{~m}$, and the remote of system 2 generates an E-field of 89 $\mathrm{dB} \mu \mathrm{V} / \mathrm{m}$ at $2 \mathrm{~m}$.

The output of the receiver was connected to a LED light that indicates if the signal from the remote was received correctly. For every frequency point, the output 


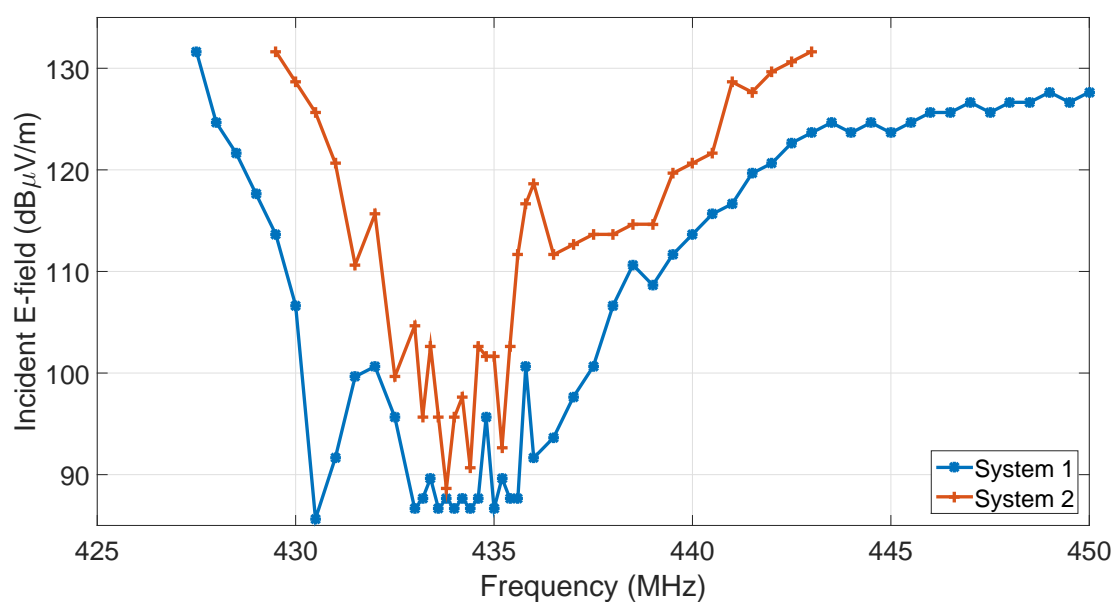

Figure 5.4: Robustness of the two RKE systems against jamming. The E-field of the interference incident on the receiver is plotted as a function of frequency.

power of the generator was being increased with steps of $1 \mathrm{~dB}$, until the signal from the key fob was not detected anymore. A picture of the actual setup can be seen in Figure 5.3b. Additional absorbers were placed on the ground to absorb the ground reflection.

The results of the measurements of system 1 and system 2 are shown in Figure 5.4. The E-field of the electromagnetic interference at the receiver, emitted by the logperiodic antenna, was calculated. The E-field strength, at which the signal from the key was not detected any more, is plotted as a function of frequency. The curve shows the robustness of the receivers against jamming as a function of frequency. For frequency points where the E-field is not plotted, we could not jam the signal because of limited output power of the signal generator.

As can be seen, system 1 has approximately a bandwidth of $5 \mathrm{MHz}$ in which it is very sensitive to interference. System 2 has a bandwidth of $4 \mathrm{MHz}$ in which the system is very sensitive. This means that a nearby transmitter in the $433 \mathrm{MHz}$ ISM band is very likely to jam the car key. This clearly shows the poor selectivity of the SRR and this results in little robustness to other systems working in the same frequency band.

For example, the two RKE systems we bought do not not work simultaneously when they are close to each other. The remote key from system 1 is able to jam the signal of system 2 if the distance to the receiver is similar, and vice versa, even though the key fobs operate at slightly different frequencies. System 1 and system 2 operate respectively on a carrier frequency of $433.85 \mathrm{MHz}$ and $433.80 \mathrm{MHz}$ [109]. 


\subsubsection{Discussion}

The poor selectivity of these SRRs make it very easy for an adversary to jam the receiver at the car, while the receiver of the adversary can still correctly detect the signal from the key fob. This is the scenario depicted in Figure 5.1. The adversary only needs a CW interference source which is out-of-band of his own receiver, but in-band of the RKE receiver.

A much robuster system would have a superheterodyne receiver, because it can achieve a high selectivity [81]. These type of receivers are actually used in many RKE applications and a typical superheterodyne receiver for this application is investigated in the next sections of this chapter. However, SRRs can still be found in particular applications for cost saving reasons.

\subsection{Analysis of pulsed interference}

Now the robustness to jamming attacks of superheterodyne receivers commonly used in RKE applications is analyzed. Of particular interest is the effect of pulsed interference on these receivers. A brief background is given on pulsed interference as compared to continuous interference and an explanation is given why it needs to be further researched.

\subsubsection{Superheterodyne receivers in RKE}

Superheterodyne receivers band-pass filter the desired signal at intermediate frequencies enabling a high selectivity. A literature study into characteristics of RKE systems from various manufacturers revealed that the demodulation in the receivers are fundamentally based on envelope detection of the received signal $[111,119,120]$. Envelope detection is the simplest form of demodulating an amplitude modulated signal and is very often used because of the low complexity. There is no synchronization required between the demodulator and the received signal (non-coherent receiver). The focus will be on the behaviour of the envelope detector under various interference scenarios.

\subsubsection{Background on pulsed interference}

Research on the matter of pulsed interference as compared to continuous interference has been reported in [121] and [122]. In both these papers, pulsed interference is compared to continuous wave $(\mathrm{CW})$ interference by assuming equal average power levels. For this reason, the amplitude of the pulsed signal is dependent on the duty cycle and it is larger than the amplitude of the CW signal. In [121], the BER of a wireless link is calculated by analyzing individually the BER during the "ON" and "OFF" states of the interference. However, the effect of transitions in received 
power when subject to pulsed interference should be included in this investigation. Any wireless receiver needs some time to adapt to new channel conditions to achieve optimal detection [123]. Pulsed interference results in a time-varying channel, which makes it difficult for the receiver to set its parameters such that the detection is optimal.

In ASK modulated signals, the information is expressed by the amplitude of the signal. To correctly decide what symbol has been sent, precise decision boundaries have to be determined [124]. The received voltage levels - after demodulation - are compared with the threshold (or decision boundary) to decide what symbol has been transmitted. A common method to estimate these boundaries is by first averaging the received power over a certain number of symbols. The determined average then provides a reference to determine the optimal decision boundaries. The estimation time of the average received power should not be so short that the estimate is inaccurate. On the other hand, it should not be so long as to make the receiver slow in adapting to new channel conditions.

\subsubsection{Simulation model of a general envelope detector}

A Matlab model of a typical envelope detector is developed to examine the effect of pulsed interference. The data signal is OOK-modulated with a data rate of $5 \mathrm{kbps}-\mathrm{a}$ data rate comparable to data rates in RKE systems - and an arbitrarily chosen signalto-noise (SNR). A part of an OOK signal with an SNR of $30 \mathrm{~dB}$ is shown in Figure 5.5. The signal levels are normalized to 1 .

The signal is demodulated by an envelope detector; it is squared and subsequently low-pass filtered with a cut-off frequency of $7 \mathrm{kHz}$. The demodulated signal is compared to the decision threshold to decide whether a logic " 1 " or " 0 " is transmitted. The decision threshold is calculated by setting it to $50 \%$ of the average received signal strength. As explained, the estimation time of this average is a trade-off between accuracy and speed. In this model, we determine the moving average over a period of 100 symbols. This period can arbitrarily be chosen, but we chose 100 symbols because this highlights the effect we would like to show.

An example of filtered data, together with the threshold estimate, is shown in Figure 5.6. In this example, the SNR was again set at $30 \mathrm{~dB}$. As can be seen, the threshold is almost equidistant from the " 1 " and " 0 " levels. Finally, the BER can be computed by comparing the received data with the transmitted data.

This model is used to analyze the effect of pulsed and continuous interference on both the threshold and the BER. First, we look at the effect of continuous noise interference on the signal. Additive white gaussian noise (AWGN) is continuously added to the modulated signal, such that the SNR is decreased to $-2 \mathrm{~dB}$. The SNR of $-2 \mathrm{~dB}$ is only chosen because this highlights the effects we would like to present. 


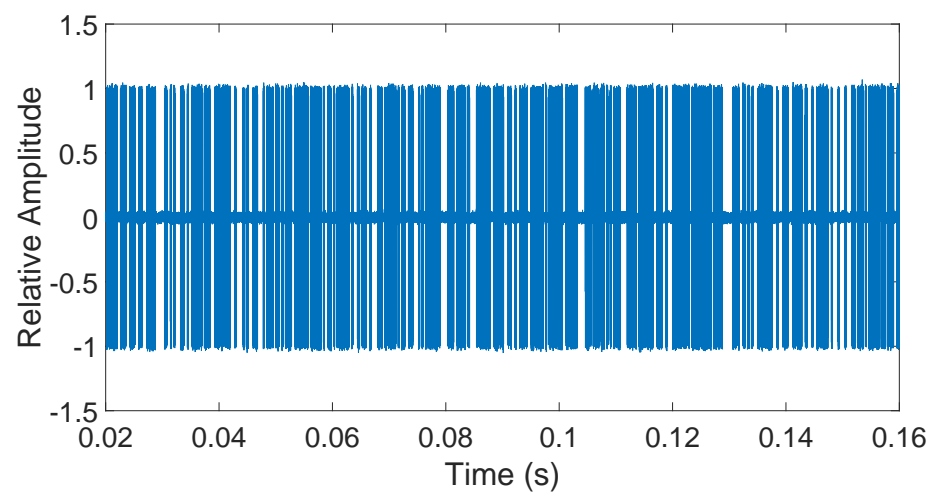

Figure 5.5: Modulated OOK signal before demodulation.

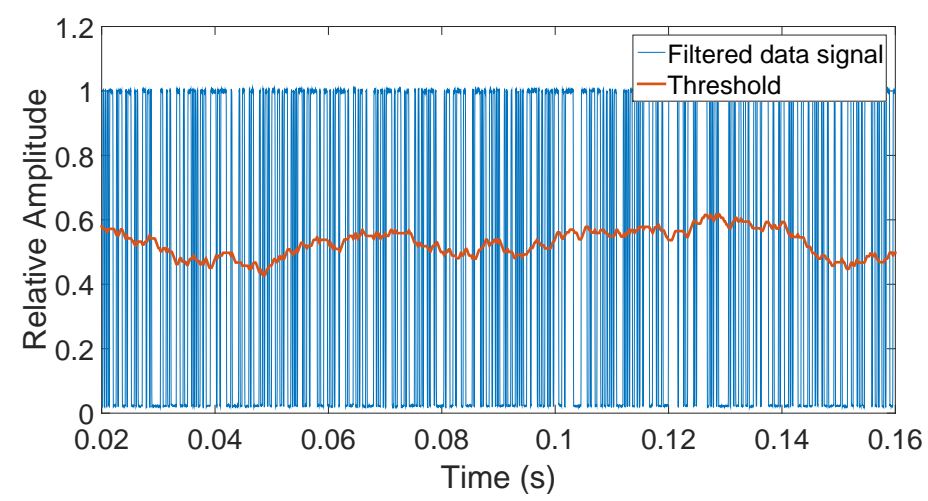

Figure 5.6: Filtered data signal, after demodulation, and the threshold estimate.

The filtered data signal (that is, after demodulation) subject to continuous noise interference is shown together with the threshold estimate in Figure 5.7. It can be seen that the signal levels are higher than in Figure 5.6. However, the threshold estimate is still set to be equidistant from the " 1 " and the " 0 " levels. The BER, calculated over 60000 bits, for this particular interference scenario is $0.7 \%$.

Next, we look at the effect of pulsed noise interference on the signal. Pulsed AWGN noise is now added to the modulated signal. The pulse repetition period was set to 200 symbols, which is exactly twice as long as the time window over which the threshold is determined. The duty cycle is set to $50 \%$. During the "ON" state the SNR is again set at $-2 \mathrm{~dB}$, and during the "OFF" state the SNR is $30 \mathrm{~dB}$. Since the duty cycle is $50 \%$ it should be clear that the average noise power is $3 \mathrm{~dB}$ less than the average noise power in the previous example. Again, the specified values in this paragraph are chosen in such a way to highlight the effects at the envelope detector 


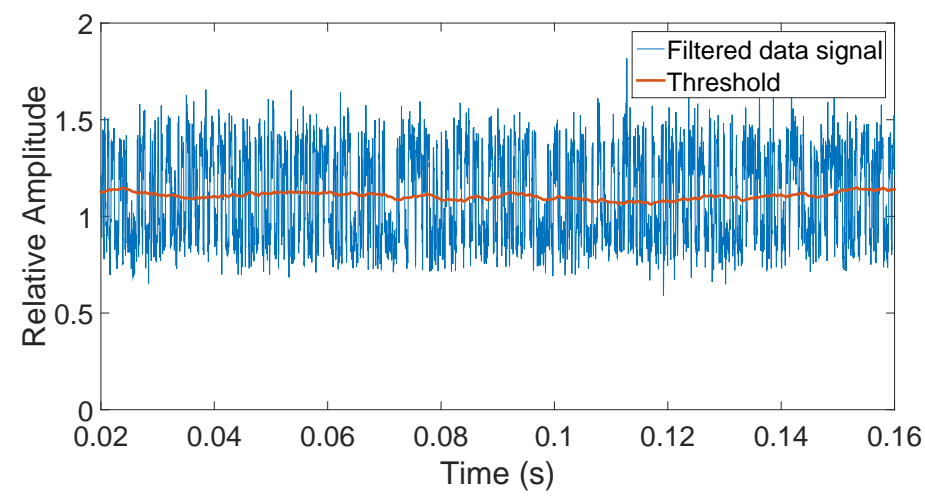

Figure 5.7: Filtered data signal, after demodulation, and the threshold estimate under continuous noise interference.

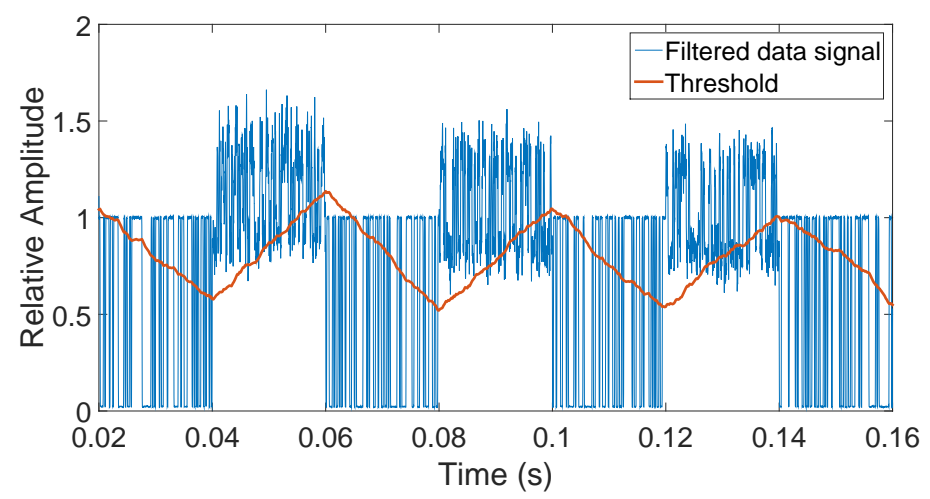

Figure 5.8: Filtered data signal, after demodulation, and the threshold estimate under pulsed noise interference.

that we graphically present in the next figures.

Figure 5.8 shows the filtered data signal subject to pulsed noise interference together with the estimated threshold. As can be seen, the threshold estimate is lagging and is not optimally adjusted to the received signal. Especially, after the interference transition from "OFF" to "ON" the threshold is not correctly set. It can easily be seen that bit errors will occur exactly at the transitions of the interference. The BER, again calculated over 60000 bits, for this interference scenario is $19.9 \%$.

From the previous two interference scenarios, we see that pulsed interference can be more effective in disrupting the wireless link of an envelope detector than continuous interference: the average noise power is lower and the BER is higher. It is more power efficient to disrupt this type of communication with pulsed interference. 


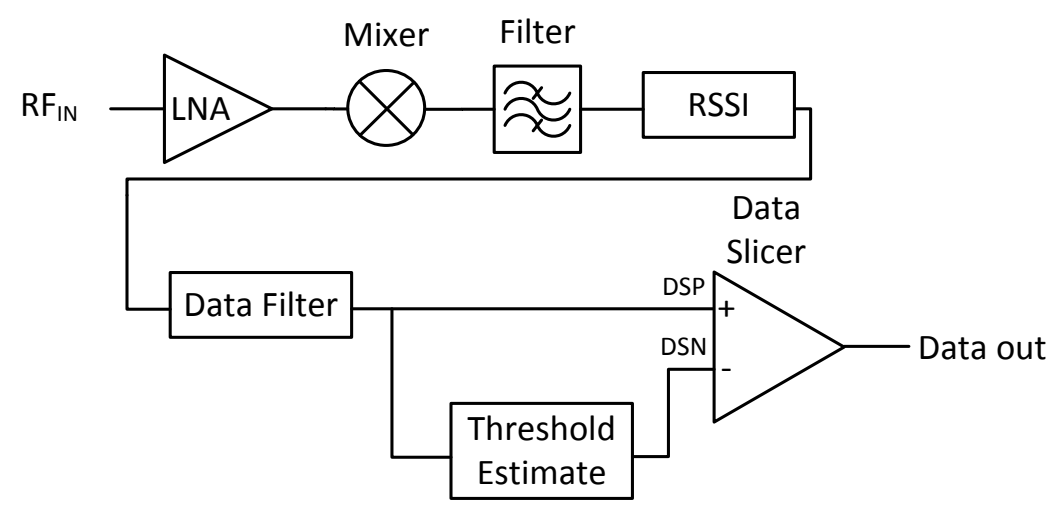

Figure 5.9: Schematic of the MAX1470 OOK receiver.

\subsection{Experimental study of an RKE receiver}

A commercial-off-the-shelf (COTS) available ASK receiver is purchased to verify the behavior described in the previous section. In this section, the behaviour of the RKE receiver is experimentally analyzed.

\subsubsection{Experimental method}

\section{Study of a COTS receiver for RKE applications}

The purchased receiver is the evaluation kit of the $433.92 \mathrm{MHz}$ MAX 1470 superheterodyne receiver $[125,126]$. This type of receiver finds its application in RKE systems. It is a low-cost heterodyne receiver tuned to $433.92 \mathrm{MHz}$ to demodulate OOK modulated signals at a maximum data rate of $5 \mathrm{kbps}$.

A simplified schematic of the receiver is shown in Figure 5.9. The received signal is first amplified by the low-noise amplifier (LNA). Next, it is mixed to an intermediate frequency of $10.7 \mathrm{MHz}$ and filtered by a band-pass filter. The received signal strength indicator (RSSI) detector demodulates the intermediate signal to baseband by producing a DC output proportional to the logarithm of the signal level with a slope of approximately $15 \mathrm{mV} / \mathrm{dB}$. The base-band signal is low-pass filtered by the data filter with a cut-off frequency of $5 \mathrm{kHz}$. This signal is finally fed to the positive input of the data slicer (DSP). The function of the data slicer is to convert the analog output of the data filter into a digital signal. The negative input of the data slicer (DSN) is the threshold estimate. The threshold estimate is computed by a taking the analog output of the data filter and connecting it to an resistor-capacitor (RC) low pass filter. This configuration averages the output of the data filter and sets the threshold at approximately $50 \%$ of the amplitude. The time constant of the RC filter is $2.35 \mathrm{~ms}$, which represents approximately $12 \mathrm{bits}$ for a data rate of $5 \mathrm{kbps}$. 


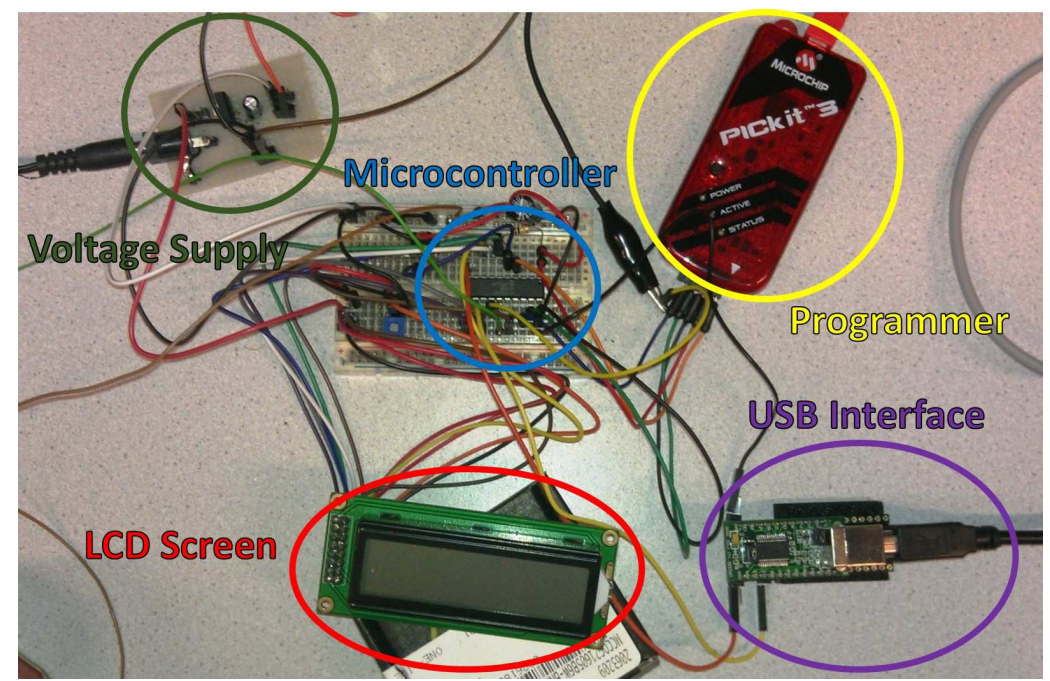

Figure 5.10: A photo of the in-house developed BER tester.

\section{Developed BER tester}

To test the effect of pulsed interference on the MAX1470 receiver, it was necessary to first develop a simple BER tester. The available commercial BER tester did not support data rates as low as 5 kbps. Therefore, the BER tester shown in Figure 5.10 was developed in-house.

This is based on a programmed PIC 16F690 microcontroller, and measures the BER between an ASK transmitter and the MAX 1470 receiver. The microcontroller's 8-bit linear feedback shift register generates pseudorandom data. One output pin of the BER tester is connected to the transmitter, and one input pin is connected to MAX 1470 receiver. For each bit, at half bit time the data is compared between the input and the output pin. If the bits do not correspond, a bit error is counted. The delay time, from generating and transmitting the bit to receiving and decoding it, is much smaller than the bit time.

The BER is calculated by dividing the number of bit errors over the number of transmitted bits. The number of transmitted bits is set at 30000 bits, which is a good trade-off between accuracy and measurement speed. The results are displayed on a 16x2 LCD screen. The BER tester is verified by some simple tests. Firstly, we coupled the output pin of the BER tester directly to the input pin. In this case, the calculated BER was $0 \%$, as expected. The next test was to connect the input pin to ground or the source voltage. In both cases the resulting BER was 50\%, as expected.

A schematic of the test set-up to measure the BER of an wireless link subject to interference is shown in Figure 5.11. It is a fully conducted set-up. The pseudorandom- 


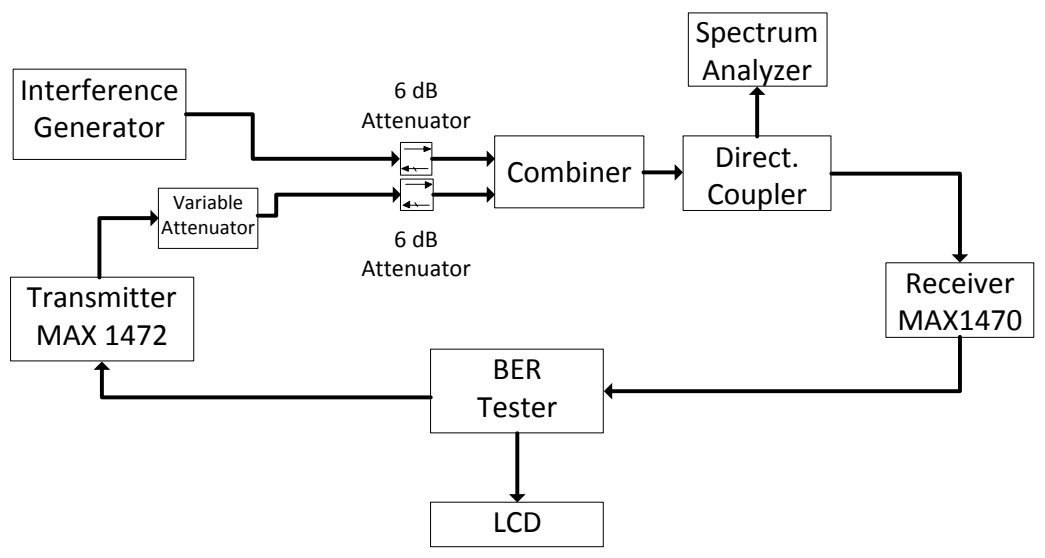

Figure 5.11: Test set-up for taking the BER measurements.

generated data from the BER tester is fed to the transmitter. The transmitter used is an evaluation kit for the MAX 1472 ASK transmitter [127]. This transmitter has a baseband data input (digital data) and it generates OOK-modulated output at 433.92 $\mathrm{MHz}$. It is capable of data rates up to $100 \mathrm{kbps}$, but we only used it at $5 \mathrm{kbps}$. We superimpose the EMI generated by a signal generator on the OOK signal using a $6 \mathrm{~dB}$ resistive power combiner. A directional coupler is used to monitor the signal levels at the input to the MAX 1470 receiver. Next, the interfered signal is received and demodulated, and the BER tester displays the BER. This set-up allows for BER measurement for many different interference scenarios generated by the interference generator. A variable attenuator was connected after the MAX1472 transmitter to attenuate the signal such that it does not saturate the receiver. The 6 - $\mathrm{dB}$ attenuators connected before the combiners function as isolators to reduce the effect of possible reflections.

\subsubsection{Experimental results}

\section{Pulsed interference versus continuous interference}

We started by monitoring the output signal of the data filter (DSP) and the threshold estimate (DSN) using an oscilloscope, see Figure 5.9. The signals were measured for various interference scenarios: no interference, continuous interference, and pulsed interference. The interference is superimposed on a desired signal. The desired signal was generated by a signal generator with a $433.92 \mathrm{MHz}$ carrier, which is $100 \% \mathrm{AM}$ modulated by a $5 \mathrm{kHz}$ square wave. The input power of the desired signal was set at $-75 \mathrm{dBm}$. The frequency of the interference was set to $433.72 \mathrm{MHz}$, and also an input power of $-75 \mathrm{dBm}$. 


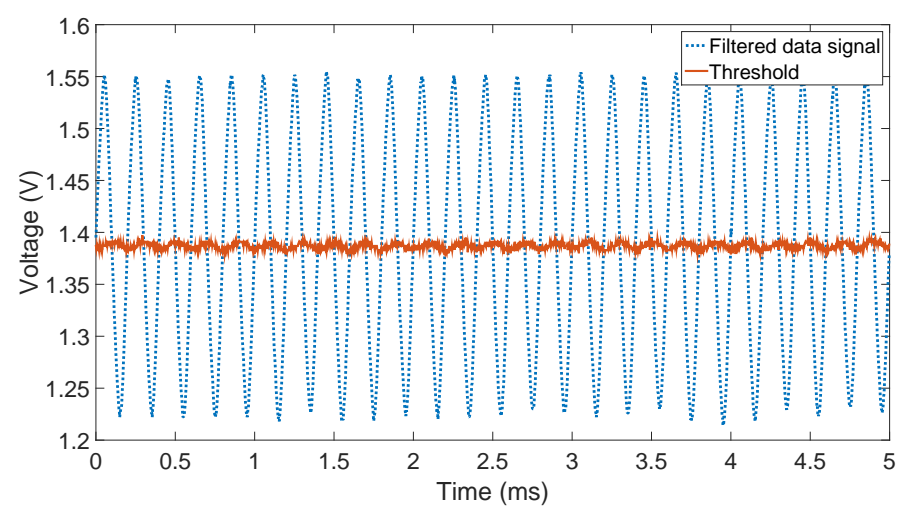

Figure 5.12: Filtered data signal and threshold with no interference.

Firstly, we measured the signals at the DSP and the DSN with no interference present. The results shown in Figure 5.12 shows the filtered data signal and the threshold estimate from the receiver. As can be seen, the threshold estimate is adjusted to the center amplitude of the filtered signal, and the receiver will have no problems recovering the data.

Next, we added continuous interference with an input power equal to the power of the desired signal. The frequency of the interference was set to $433.72 \mathrm{MHz}$. The filtered data signal and threshold estimate in this interference scenario is shown in Figure 5.13.

The filtered data signal is increased up to the continuously present energy of the interference, and the swing in the data signal is much smaller, compared to the swing in the data signal in Figure 5.12, due to the logarithmic behavior of the RSSI. However, the threshold estimate adapts easily to this new channel condition and the threshold is again adjusted to the center amplitude of the filtered signal. Again, the receiver will have no problems recovering the data.

Finally, we added pulsed interference to the desired signal. The carrier frequency is set at $433.72 \mathrm{MHz}$, the pulse repetition frequency is set at $500 \mathrm{~Hz}$ with a duty cycle of $50 \%$, and again the power level is set at $-75 \mathrm{dBm}$. The results are shown in Figure 5.14 .

As can be seen, the threshold estimate is constantly changing due to the varying channel conditions, caused by the pulsed interference. As a result, the receiver is no longer able to correctly recover the data. Figure 5.14 clearly shows the problems that occur at this envelope detector under pulsed interference scenarios, whereas continuous interference scenarios from Figure 5.13 does not show significant problems. 


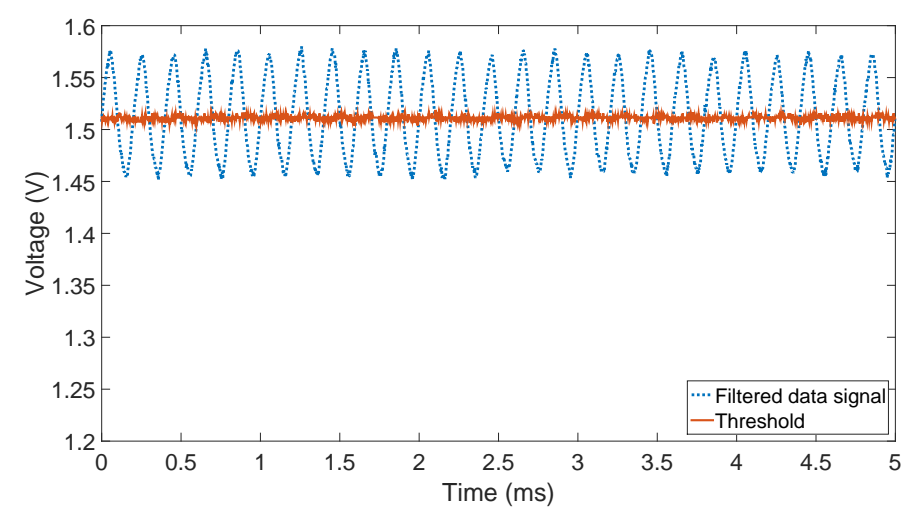

Figure 5.13: Filtered data signal and threshold with CW interference.

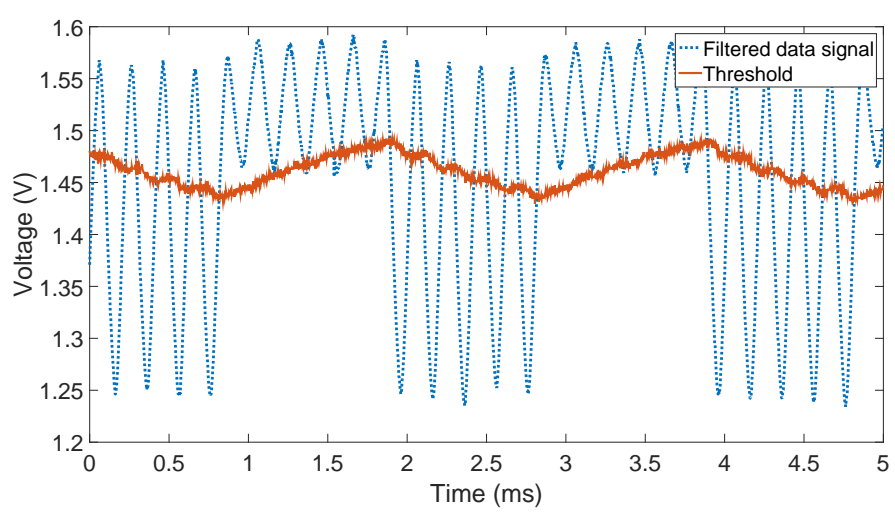

Figure 5.14: Filtered data signal and threshold with pulsed interference.

\section{BER measurements}

The previously described effect exhibited by the receiver was quantified by a BER measurement as shown in Figure 5.11. The results are shown in Figure 5.15 in which the BER is presented on a linear scale - since for now we are interested in a BER exceeding $1 \%$. The power of the desired signal at the input of the receiver was measured as $-65 \mathrm{dBm}$. The presented BER curves are a function of the average power level of the interference. The pulsed interference was set at a PRF of $1 \mathrm{kHz}$ with a $50 \%$ duty cycle.

As can be seen, for interference power levels below $-72 \mathrm{dBm}$ the pulsed interference is more disruptive than the continuous interference. However, for interference power levels exceeding $-72 \mathrm{dBm}$, the desired signal is completely masked and $\mathrm{CW}$ is more disruptive since it is active $100 \%$ of the time. In the low power region, namely below 


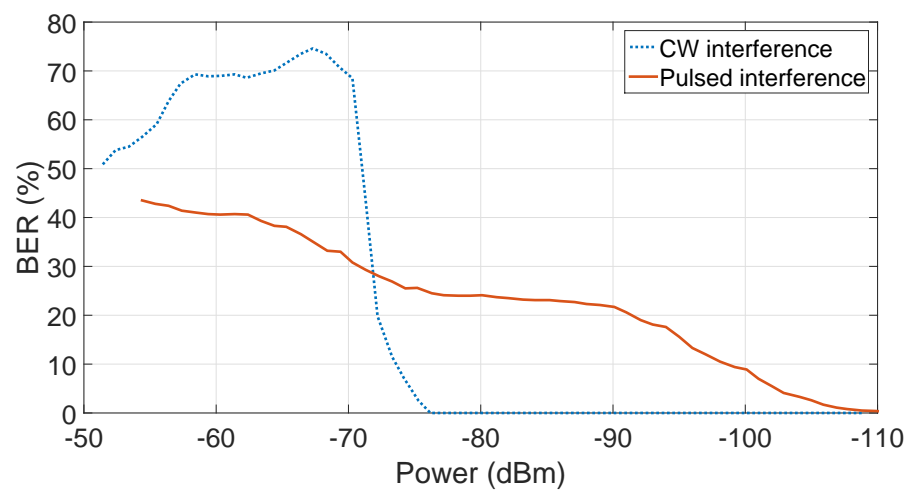

Figure 5.15: BER curves for CW interference and pulsed $\mathrm{CW}$ interference as a function of interference power.

$-72 \mathrm{dBm}$, pulsed interference increases the BER from interference power levels as low as $-110 \mathrm{dBm}$, whereas the continuous interference has no effect until the power level exceeds $-75 \mathrm{dBm}$. Of course, all the mentioned interference power levels are relative to the power level of the desired signal.

The fact that the BER exceeds $50 \%$ is a feature of this typical receiver and is related to an inverting behavior of the receiver under certain conditions. It is related to the logarithmic behaviour of the RSSI in combination with an existing beat if both the carrier of the desired signal and the CW interference are at the input of this RSSI. However, it is beyond the scope of this thesis to fully explain this effect.

\section{Pulse repetition frequency}

The results presented in Figure 5.15 compare continuous interference to pulsed interference with a PRF of $1 \mathrm{kHz}$. It is easily understood that the PRF is an important parameter affecting the effectiveness of the pulsed interference. Pulsed interference with a very high PRF will be filtered out by the data filter, but interference with a very low PFR appears to be almost equal to continuous interference.

The optimal PRF is dependent on the averaging time of the threshold estimate. Interference with a short pulse repetition period - shorter than the averaging time of the receiver - does effect the threshold at a high rate, but if the pulse repetition period becomes too short it is not greatly effecting the threshold estimate any more, because the pulsing effect is averaged out.

We measured the sensitivity of the MAX1470 receiver [125] towards the PRF by varying it from $\mathrm{DC}$ to $10 \mathrm{kHz}$ with steps of $0.25 \mathrm{kHz}$ with a $50 \%$ duty cycle. The power of the desired signal was set at $-65 \mathrm{dBm}$, and the power of the interference was set at $-93 \mathrm{dBm}$. The results are shown in Figure 5.16. 


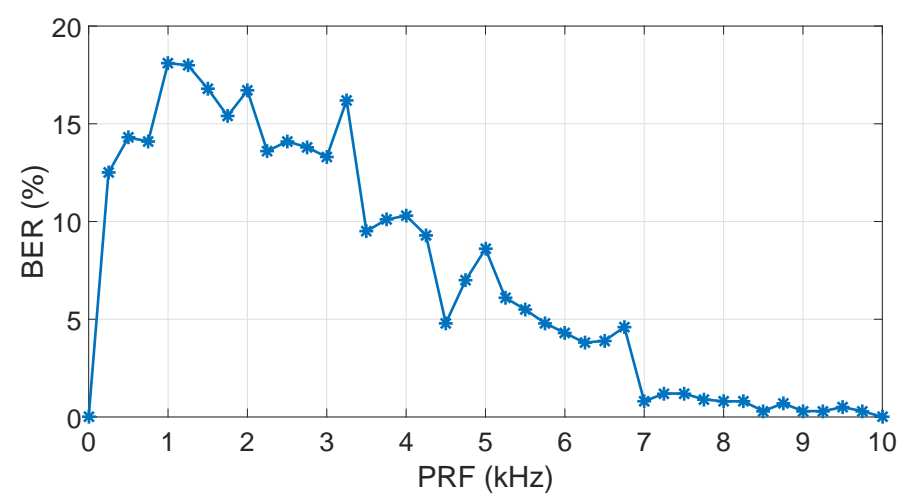

Figure 5.16: BER as a function of pulse repetition frequency.

From DC to $1 \mathrm{kHz}$, it shows a very rapid increase in the BER from $0 \%$ to $18 \%$. The optimum PRF is shown to be at $1 \mathrm{kHz}$, and from here on the BER is decaying for increasing PRF. As mentioned in Section 5.4.1, the RC-time of the threshold estimate of the MAX1470 receiver is $2.35 \mathrm{~ms}$, and apparently the optimal pulse repetition period to disrupt this receiver is $1 \mathrm{~ms}$. For PRF exceeding $5 \mathrm{kHz}$ the data filter also starts to attenuate the pulsed interference. We can see that the PRF exceeding $7 \mathrm{kHz}$ is hardly any more effective in jamming the wireless link than continuous interference, that is, interference with a PRF of $0 \mathrm{~Hz}$.

\subsection{Improved receiver type}

\subsubsection{Vulnerability of receiver against pulsed interference}

Key of the hacking technique described in [114] is that the receiver of the RKE system is jammed, while the intruder's receiver is able to receive the transmitted code from the key fob. From the previous sections, it can be concluded that typical RKE receivers are highly vulnerable to pulsed noise interference as compared to continuous noise interference.

The vulnerability of the RKE system can be exploited by having a receiver which is robust against this type of interference. In this case, it is possible for an adversary to jam the receiver of the RKE receiver with an EMI source generating pulsed noise, while the adversary is able to receive the code from the key with a robust receiver.

High power continuous interference would jam the receiver of the RKE system as well, as can be seen from Fig. 5.15, but it would most likely also jam the intruder's receiver. In this case, the hacking technique described in [114] would not be able to work. 


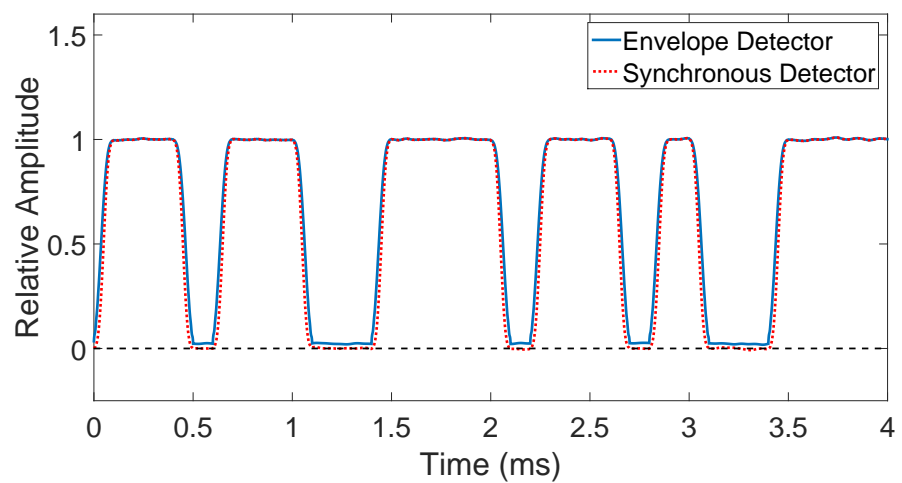

Figure 5.17: Filtered data signal of both envelope and synchronous detector with an SNR of $30 \mathrm{~dB}$.

\subsubsection{Synchronous detector}

The vulnerability of RKE systems described in the previous section can be avoided by having receivers which are robust against pulsed interference. Most often the receivers in RKE systems use envelope detectors, but the message signal in an AM signal can also be recovered by a synchronous (or coherent) detector [128].

A synchronous detector demodulates the AM signal by multiplying the incoming signal with a copy of the carrier frequency - hereby mixing the signal to DC - and subsequently low-pass filtering the product. Synchronous detectors have many advantages over an envelope detector, among which an improved sensitivity [128]. The reason that most often envelope detectors are used is the simplicity of the receiver design, resulting in an attractive economic factor, as compared to the readily available synchronous detectors. The increased complexity of a synchronous detection is due to the generation of a local sinusoidal wave having exactly the carrier frequency of the incoming signal. The synchronization between the local sinusoidal wave and the carrier needs to be maintained in time.

\subsubsection{Simulations of improved performance}

We developed a Matlab model of a synchronous receiver, to compare the performance under interference scenarios to the envelope detector modelled in Section 5.3.3. The synchronous detector multiplies the received signal with a local oscillator which is frequency and phase locked with the carrier frequency of the incoming signal. The product is then low-pass filtered by the same data filter as used in Section 5.3.3.

In Fig. 5.17, we show a part of the demodulated signal of both the envelope and synchronous detector. As can be seen, under these high SNRs of $30 \mathrm{~dB}$ the 


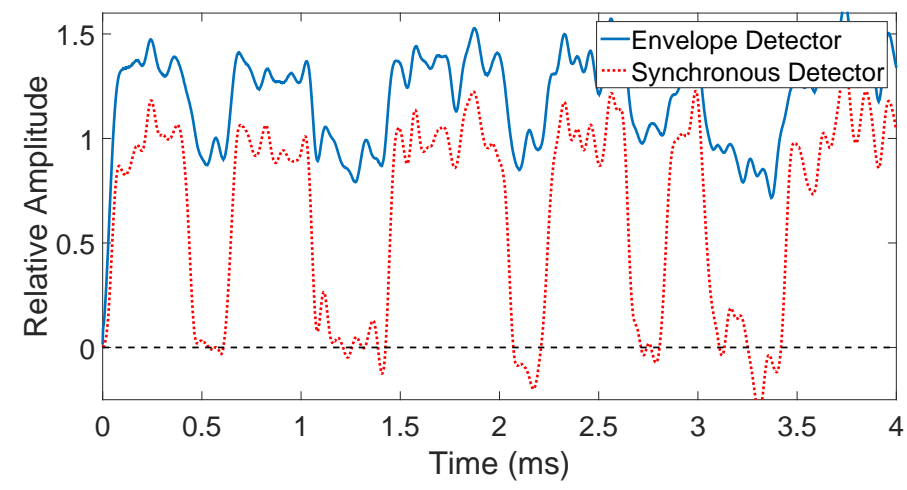

Figure 5.18: Filtered data signal of both envelope and synchronous detector with an SNR of $-2 \mathrm{~dB}$.

Table 5.1: Simulated BER of an envelope and synchronous detector under continuous and pulsed noise interference scenarios.

\begin{tabular}{|l|c|c|}
\hline Detector & $\begin{array}{c}\text { BER (\%) with } \\
\text { continuous interference }\end{array}$ & $\begin{array}{c}\text { BER (\%) with } \\
\text { pulsed interference }\end{array}$ \\
\hline \hline Envelope & 0.7 & 19.9 \\
\hline Synchronous & 0.1 & 0.1 \\
\hline
\end{tabular}

filtered data signals are comparable. Next, we lowered the SNR of the signal to -2 $\mathrm{dB}$ and compared the demodulated signals again in Fig. 5.18. Now we can clearly see the difference. The low SNR results in a DC offset in the demodulated signal of the envelope detector. It is exactly this offset that causes the threshold estimate to drift in the case of pulsed interference. The demodulated signal of the synchronous detector does not show a DC offset, so the threshold estimate will not drift in the case of pulsed interference.

The difference between the envelope and synchronous detector can be quantified by a BER simulation. Again, as in Section 5.3.3, we computed the BER over 60000 bits at an SNR of $-2 \mathrm{~dB}$, for both continuous and pulsed interference. The results are summarized in Table 5.1. This clearly shows that the synchronous detector is not affected by the pulsed noise interference as the envelope detector is. Therefore, we suggest to implement receivers in RKE with synchronous detectors instead of envelope, to improve the robustness of these systems against jamming attacks. 


\subsection{Summary and conclusions}

In this chapter, we have investigated the vulnerability of RKE systems against jamming attacks. The system is increasingly secured against hacking attacks by use of encryption and code algorithms. However, there are effective hacker attacks that rely on jamming the wireless link from the key fob to the receiver, while the attacker is able to receive the signal from the key fob.

Firstly, the selectivity of two purchased RKE systems was analyzed. It is found that these low-cost systems were equipped with super regenerative receivers which showed a poor selectivity. This poor selectivity enables an adversary to easily jam this receiver.

Next, the vulnerability of typical superheterodyne receivers used in RKE applications was investigated. The robustness of these receivers against pulsed interference as compared to continuous interference is analyzed. Most RKE receivers are equipped with envelope detectors and it is shown, both through simulations and measurements, that these type of detectors are very easily jammed by pulsed interference. The PRF of the interference does impact the effectiveness of a jamming attack, and for it to be effective, the PRF should be at least below the data rate of the wireless link.

An improved RKE system would use a receiver with a synchronous detector. Pulsed noise interference is not more effective in jamming a synchronous detector as compared to continuous noise interference. RKE systems with synchronous receivers would be less vulnerable against specific hacking attacks.

This chapter and Chapter 4 discussed the jamming interference mechanism. The next chapter will further investigate the saturation of a wireless receiver, which is one of the interference mechanism as defined in Chapter 3. 


\section{Chapter 6}

\section{Blocking and desensitization}

In this chapter, the effects of a saturated receiver is further investigated. The focus is on the blocking and desensitization effects of an accompanying high-power interferer with the desired signal. The work presented in this chapter is currently being prepared for submission to the IEEE Transactions on EMC.

\subsection{Rationale}

As discussed in Chapter 2 and Chapter 3, the most likely attack against wireless communication is a jamming attack. For this reason, many anti-jamming measures were developed, with spread spectrum techniques being one of the most common techniques. Even though these techniques do increase the robustness against jammers, they are useless against EM interference mechanisms such as damaging or blocking the receiver by a high-power interferer. In this chapter, the effects of a blocker as introduced in Chapter 3.4 on the performance of a wireless receiver is investigated.

Whereas a jamming signal needs to be in-channel of the communication signal to cause a DoS, this is not per se necessary for a blocker that saturates a receiver. Out-of-band (OOB) blockers can be as detrimental to the performance of a receiver as an in-band blocker. As a result, wideband wireless receivers, typically employed by spread spectrum systems, are susceptible to OOB blockers. For this reason, it is important to investigate the saturation levels of the receiver over a wideband, as presented in Chapter 3.

In most standards it is required that a receiver complies with a certain blocking template. For instance, in TETRA, it is described in the testing specifications that a desired signal, only $3 \mathrm{~dB}$ above the sensitivity level, should be correctly received; even if accompanied by an out-of-band (OOB) blocker as large as $-25 \mathrm{dBm}$ [80]. This test has a pass or fail outcome and gives no information on the power levels at which 
the receiver starts to cause problems or lose performance.

In this chapter, a method is presented that can be used to quantify and measure the performance of a wireless system under blocking conditions. The end-to-end performance of a wireless system can be quantified by the bit-error-rate (BER) of the received digital signal. The receiver performance is estimated by investigating the behaviour of the front end under blocking conditions, and translate these characteristic to the expected BER.

In Section 6.2, the effects of a blocker on a receiver are discussed. These effects are experimentally verified on a wideband LNA in Section 6.3. In Section 6.4, the negative effects are translated to the loss in performance of a wireless system by simulating the bit error probability. Possible improvements for wireless systems against blockers are discussed in Section 6.5. Finally, in Section 6.6, the conclusions and a summary of this chapter are presented.

\subsection{Blocking mechanisms}

In this section, the effects a blocker have on a wireless receiver are briefly summarized. In the first section, we look into the non-linear effects of the receiver, and in the next section we describe the main effects that contribute to the desensitization of the receiver.

\subsubsection{Non-linear effects}

As described in Chapter 3.4.2, the input/output characteristic of a non-linear memoryless system can be approximated by

$$
y(t) \approx \alpha_{1} x(t)+\alpha_{2} x^{2}(t)+\alpha_{3} x^{3}(t) .
$$

In this equation $\alpha_{1}$ is considered to be the desired small-signal gain. A fully accurate description of a physical non-linear device would require an infinite number of terms, but as will be shown, the main non-linearity effects can be described by the third-order term. The main effects of non-linearities are harmonic distortion, gain compression, cross modulation and intermodulation. These effects will now be briefly summarized; a detailed explanation is presented in [81].

\section{Harmonic distortion}

Sinusoids applied to a non-linear system will result in an output that exhibits frequency components which are an integer multiples of the input frequency. The output components which are integer multiples of the input frequency are called "harmonics". Even-order non-linearities also introduce DC offsets. 


\section{Gain compression}

The effect of gain compression is already described in Chapter 3. If we consider the input signal to be

$$
V_{i}=V_{d} \cos \omega_{d} t+V_{b} \cos \omega_{b} t,
$$

where $V_{d} \cos \omega_{d} t$ is the small desired signal and $V_{b} \cos \omega_{b} t$ is the blocker, then the small-signal gain of the desired signal can be expressed as

$$
G=\alpha_{1}-\frac{3}{2} \alpha_{3} V_{b}^{2}
$$

The gain behaviour described in Eq. (6.3) is a good approximation for weakly nonlinear systems. A more accurate estimation of the compressed gain due to the blocker would include the fifth and the seventh order, but for now this model suffices.

The level of the blocker that requires a $-1 \mathrm{~dB}$ gain compression can be calculating by setting [129]

$$
20 \log _{10}\left(1+\frac{3}{2} \frac{\alpha_{3}}{\alpha_{1}} V_{b}^{2}\right)=-1
$$

and it can be found that

$$
V_{b}=0.269 \sqrt{\frac{\left|\alpha_{1}\right|}{\left|\alpha_{3}\right|}} .
$$

Similary, it can be found that the blocker level required to cause a $-3 \mathrm{~dB}$ compression is

$$
V_{b}=0.442 \sqrt{\frac{\left|\alpha_{1}\right|}{\left|\alpha_{3}\right|}} .
$$

\section{Cross modulation}

Another phenomenon that arises when a large interferer, blocker, accompanies the desired signal is the transfer of modulation from the interferer to the desired signal. This can clearly be seen from Eq. (6.3); if the amplitude of $V_{b}$ varies this will be directly translated to the amplitude of the desired signal at the output of the system.

\section{Intermodulation}

Intermodulation occurs if two interferers accompany the desired signal. This can result into spurious responses at the output which are not obvious from only harmonic distortion or gain compression. Two interferers at $\omega_{1}$ and $\omega_{2}$ can result into output products which arise from mixing of the two input components. Of particular interest are the third-order intermodulation products at $2 \omega_{1}-\omega 2$ and $2 \omega_{2}-\omega_{1}$. The thirdorder intermodulation products are often the main culprits in corrupting the desired signal [81]. 


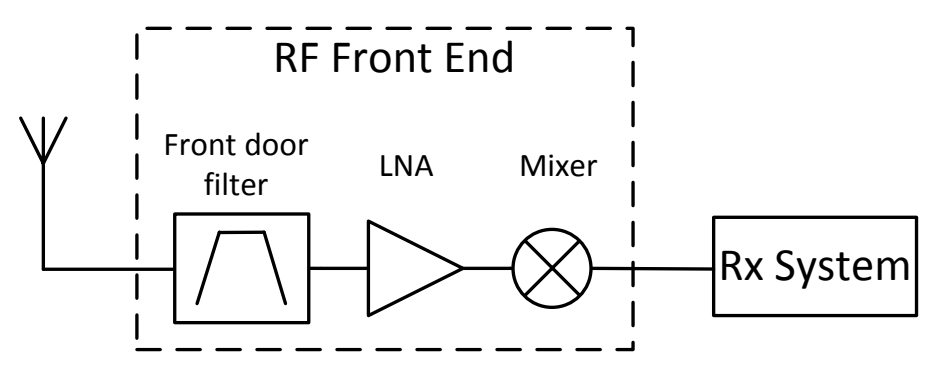

Figure 6.1: Block diagram of the front end of a receiver.

\subsubsection{Desensitization}

Desensitization is defined as the phenomenon that lowers the SNR at the output of the receiver, because of an increase in the receiver noise figure due to the blocker. This effect directly degrades the sensitivity of the receiver. For digital systems, the sensitivity is defined as the minimal received signal power that is necessary to receive the data with a BER under a certain threshold. For example, in TETRA the data needs to be received with a BER smaller than $3 \%$.

The SNR at the output of a receiver is dependent on the noise figure and is given by

$$
S N R_{\mathrm{OUT} \mid \mathrm{dB}}=S N R_{\mathrm{IN} \mid \mathrm{dB}}+F_{\mathrm{TOTAL} \mid \mathrm{dB}},
$$

where $F_{\text {TOTAL }}$ is the total noise figure of the receiver.

The degrading effects of OOB blockers normally happen at the RF front end, because an OOB blocker is mostly likely suppressed after the mixer due to the sharp filtering at IF. The front end consists of all the circuitry from antenna to mixer, with mixer included. The noise figure of the receiver as depicted in Figure 6.1 is given by

$$
F_{\mathrm{TOTAL}}=F_{\mathrm{FE}}+\frac{F_{\mathrm{RX}}-1}{G_{\mathrm{FE}}},
$$

where $F_{\mathrm{FE}}$ and $G_{\mathrm{FE}}$ are respectively the noise figure and the conversion gain of the front end.

The two main principles which increase $F_{\text {TOTAL }}$ are:

1. a decrease in the gain of either the LNA or mixer due to saturation. As a result the noise contribution of the following receiver blocks are elevated;

2. an increase in $F_{\mathrm{FE}}$ due to saturation of the stages at $\mathrm{RF}$.

Normally, the second principle is the principal cause of desensitization, especially for receivers with a high gain LNA $[91,130,131]$. The gain compression of a front end is 


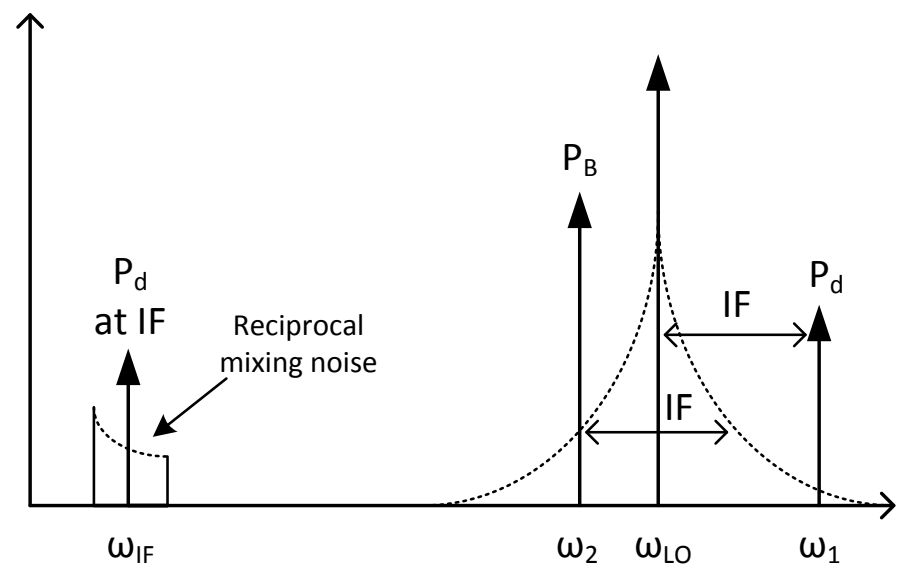

Figure 6.2: Reciprocal mixing where $\mathrm{P}_{\mathrm{B}}$ and $\mathrm{P}_{\mathrm{d}}$ are respectively the power of the blocker and the desired signal.

normally dominated by the mixer, since the LNA amplifies the received RF signal [81]. If the LNA would firstly clip, the noise contribution of the mixer is also elevated due to the reduced gain of the LNA.

The noise figure of both the LNA and active mixers is increased at high signal levels because of non-linearities which cause noise power at frequencies out-of-band to be converted in-band. Thus the single stage noise figures of active circuits designed for optimal noise figure performance will degrade under blocking conditions.

Another important principle that increases the noise figure of a mixer is the reciprocal mixing of the interferer with the phase noise of the local oscillator (LO). Any present blocker, even if it does not cause gain compression, will mix with the LO phase noise and deposit additive noise in-channel at IF. This phenomenon is graphically explained in Figure 6.2.

The effect of reciprocal mixing diminishes fast if the frequency offset between the blocker and the LO is large. Another obvious improvement is to generate LO signals with very low phase noise.

The noise figure behaviour of the front end under blocking conditions is often estimated to increase linearly with the power level of the interfering signal $[91,130$, 131], that is

$$
F_{\mathrm{FE}}=F_{\mathrm{FE}, 0}\left(1+\left(\frac{V_{b}^{2}}{V_{o}^{2}}\right)\right),
$$

where $F_{\mathrm{FE}, 0}$ is the small signal noise figure, $V_{b}$ is the blocker voltage and $V_{o}$ is the 
value of $V_{b}$ which doubles the noise factor of the front end.

\subsection{LNA characterization}

In this section, the results will be presented of the characterization of a COTS LNA.

\subsubsection{Experimental set-up}

The experimental set-up is shown in Figure 6.3. The set-up is very comparable to the set-up described in Chapter 3.5 to measure the $P_{O B}$, which is the input signal level of the blocker to compress the small signal gain with $1 \mathrm{~dB}$.

The device-under-test (DUT) that we used is a wideband LNA, but any front end, including mixer, could be used for the measurements that will be presented in this section. The effects of reciprocal mixing is not included in this experimental study, since there is no mixer involved. The DUT is the RF Bay LNA-1440 [132], which is a $40 \mathrm{~dB}$ gain wideband high dynamic range LNA operating at the frequency range from $10 \mathrm{kHz}$ to $1400 \mathrm{MHz}$.

A BPF is connected to the output of the blocker generator to suppress excess wideband noise and harmonics generated by the signal generator. After the DUT a band reject filter (BRF) with over $50 \mathrm{~dB}$ attenuation is connected to suppress the OOB blocker. In this way, the effect of the blocker is isolated to the LNA and has no impact on the internal receiver of the vector signal analyzer (VSA). The goal of this set-up is to measure the impact of the blocker, which is a CW, solely on the LNA.

The generator for the desired signal is the Agilent E4438C Vector Signal Generator (VSG) capable of transmitting digital-modulated signals. The signal analyzer is the Agilent Vector Signal Analyzer (VSA) N9020A MXA. This is an advanced analyzer capable of measuring many signal parameters, such as the received constellation diagrams, error vector magnitude (EVM) and the SNR. For the blocker the Rhode \& Schwarz SMS generator is used.

\subsubsection{Gain compression}

The small signal gain compression was measured in a similar way as presented in Chapter 3.5. The desired signal was set at $394 \mathrm{MHz}$ with an input power of -60 $\mathrm{dBm}$ at the input of the LNA. The blocker power at the input was varied from -35 $\mathrm{dBm}$ to $-5 \mathrm{dBm}$ with steps of $1 \mathrm{~dB}$, and simultaneously the gain of the desired signal was monitored. This measurement was repeated for various frequencies of the $\mathrm{CW}$ blocker, i.e. at 380, 390, 395, 400, and $410 \mathrm{MHz}$.

The resulting small signal gain curves are presented in Figure 6.4. As can be seen, the small signal gain curves are very similar and independent on the frequency of 


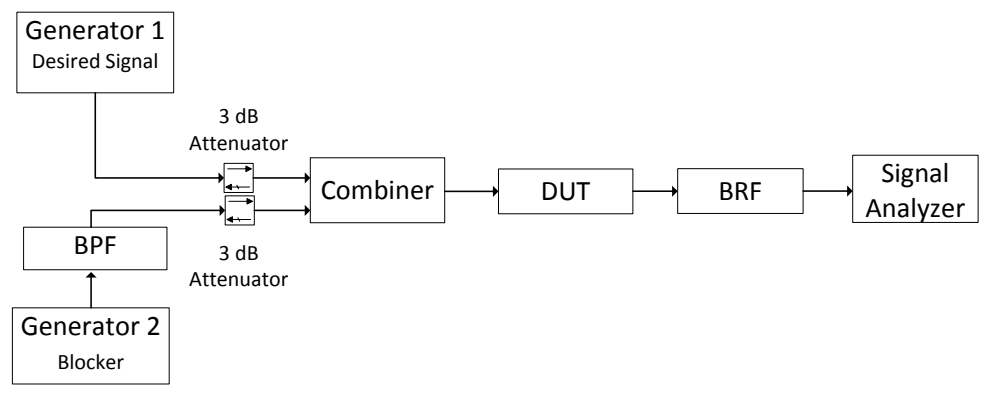

Figure 6.3: Test set-up for the LNA characterization.

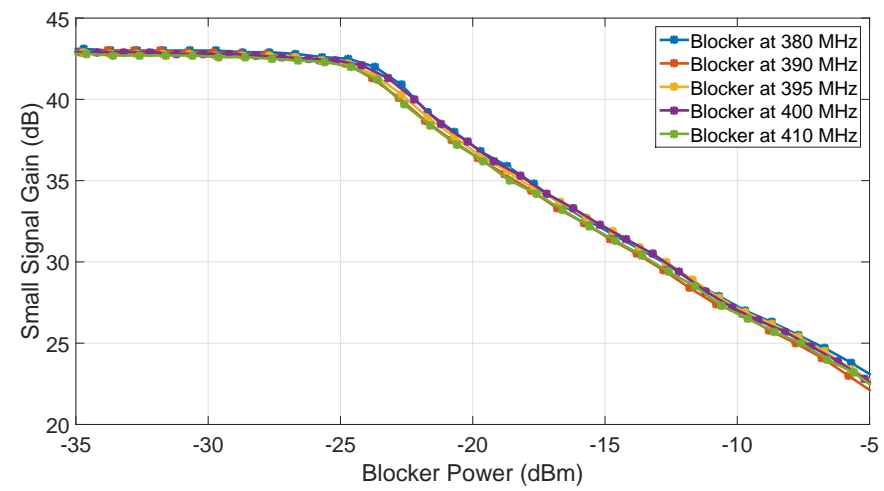

Figure 6.4: Small signal gain as a function of blocker power at various frequencies.

the blocker. This is an important observation, because, as expected, for a wideband receiver the small signal gain compression does not seem to be dependent on the frequency of the blocker.

Next, we compared the measured small signal gain curve with the analytical model from (6.3). The small signal gain, i.e. the $\alpha_{1}$ value, is $43 \mathrm{~dB}$. The value of $\alpha_{3}$ was determined for $1 \mathrm{~dB}$ compression using (6.5), and secondly, an $\alpha_{3}$ was determined for 3 $\mathrm{dB}$ compression using (6.6). The measured values of $V_{b}$ for $1 \mathrm{~dB}$ and $3 \mathrm{~dB}$ compression, with the blocker at $410 \mathrm{MHz}$, are respectively $19 \mathrm{mV}$ and $23 \mathrm{mV}$, corresponding to input blocker power levels of $-24.5 \mathrm{dBm}$ and $-22.6 \mathrm{dBm}$

In Figure 6.5 the measured gain curve with a blocker at $410 \mathrm{MHz}$ together with the two models are plotted. The first model adjusted the value of $\alpha_{3}$ to match the 1-dB compression point $\left(\alpha_{3,1-d B}\right)$, the second model adjusted the value of $\alpha_{3}$ to match the 3 -dB compression point $\left(\alpha_{3,3-d B}\right)$.

At the onset of compression the 1-dB compression model shows a good fit, but for 


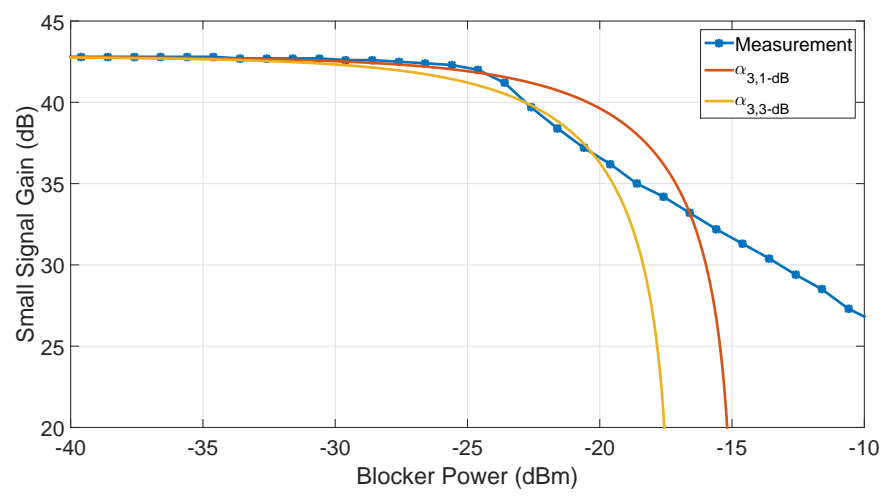

Figure 6.5: Small signal gain as a function of blocker power at $410 \mathrm{MHz}$ and the models with $\alpha_{3}$ fixed for 1-dB compression (red curve) and 3-dB compression (yellow curve).

increasing blocker power the model underestimates the compression with a maximum $3 \mathrm{~dB}$. From $-16 \mathrm{dBm}$ the gain model highly overestimates the compression.

The 3-dB compression model shows an onset of the gain compression before actual compression occurs. However, it does show a better fit for blocker power levels from $-23 \mathrm{dBm}$ up to $-19 \mathrm{dBm}$. Again, this gain model also overestimates the compression for higher power levels of the blocker.

To get a better fit between the measurements and the model, higher-order polynomials, such as the fifth and the seventh order, should be added to the approximation presented in (6.1).

\subsubsection{Distorted spectrum}

The output spectrum of the LNA was monitored for various blocker power levels. Again, the desired signal was set at $394 \mathrm{MHz}$ with an input power level of $-60 \mathrm{dBm}$. The blocker was set at $410 \mathrm{MHz}$ at 4 different power levels (off, $-42,-32$, and -22 $\mathrm{dBm})$. The output spectrum was measured from 380 to $420 \mathrm{MHz}$ with a resolution bandwidth of $3 \mathrm{kHz}$. The results are presented in Figure 6.6.

In Figure 6.6a the blocker is switched off; at $394 \mathrm{MHz}$ the small signal can be seen and around $410 \mathrm{MHz}$ there is a dip in the noise due to the BRF. What is interesting about Figure $6.6 \mathrm{c}$ is that we can see a distorted output spectrum even though the blocker power level is still well below the $1-\mathrm{dB}$ compression level $(-24.5 \mathrm{dBm}$.) At a blocker level of $-22 \mathrm{dBm}$ the there are many strong spurious responses and the noise level increases.

This spurious responses can again mix with the LO if there would be a mixer, 


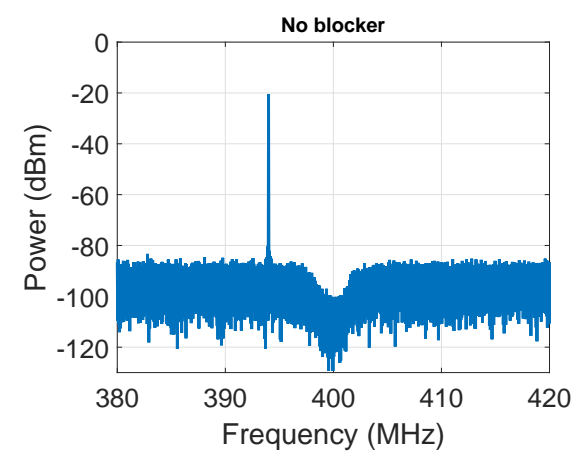

(a)

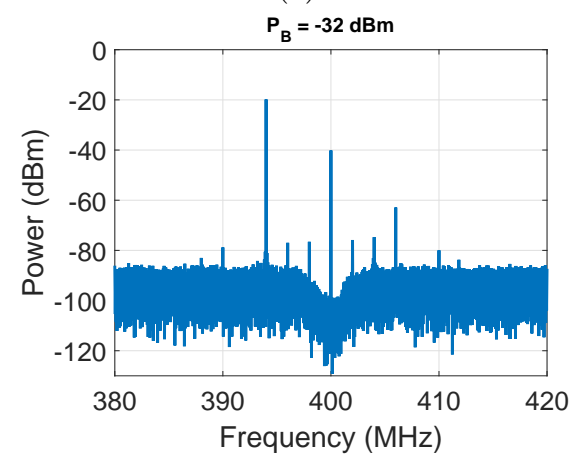

(c)

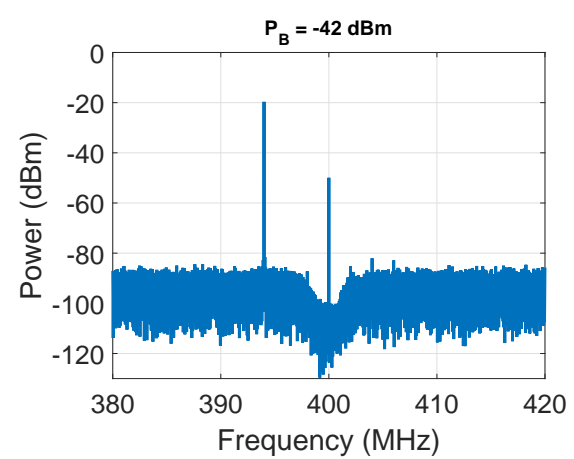

(b)

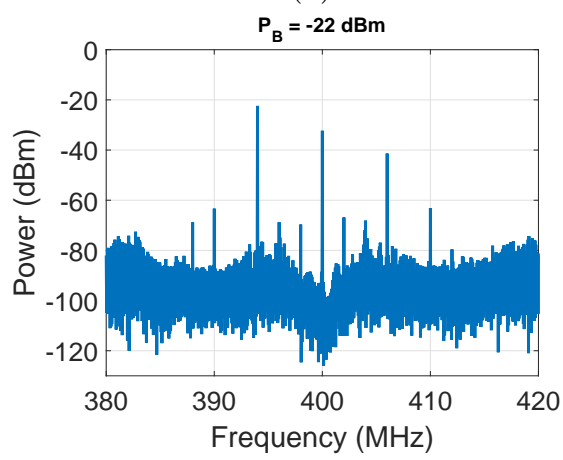

(d)

Figure 6.6: Output spectrum of the LNA under various blocker power conditions.

resulting in many more spurious responses (in-band and OOB) and additional reciprocal mixing. These effects could be mitigated by implementing a BPF between the LNA and the mixer.

\subsubsection{Blocker noise figure}

With the set-up presented in Figure 6.3, the noise figure of the LNA was measured. In the next section, it is first explained in what way the noise figure was measured.

\section{Method}

The desired signal generator transmits a QPSK signal with an arbitrary symbol rate. The VSA demodulates the signal and and calculates the normalized EVM as described in $[133]$.

Under additive white gaussian noise (AWGN) conditions this EVM can be directly related to SNR. For systems, which are sampled at data rate, the SNR is equal to $\frac{E_{s}}{N_{0}}$, 
where $E_{s}$ is the symbol energy and $N_{0}$ the noise power spectral density [134]. The relation among these metrics are [134]

$$
E V M_{\mathrm{RMS}}=\left[\frac{1}{S N R}\right]^{\frac{1}{2}}=\left[\frac{N_{0}}{E_{s}}\right]^{\frac{1}{2}} .
$$

The SNR measured by the VSA is

$$
S N R_{\text {measured } \mid \mathrm{dB}}=S N R_{\text {in } \mid \mathrm{dB}}-F_{\text {system } \mid \mathrm{dB}}
$$

where $S N R_{\text {in }}$ is the SNR at the input of the LNA, and $F_{\text {system }}$ is

$$
F_{\mathrm{system}}=F_{\mathrm{LNA}}+\frac{F_{\mathrm{VSA}}-1}{G_{\mathrm{LNA}}},
$$

where $F_{\mathrm{VSA}}$ is the noise figure of the VSA which is measured to be approximately 9 dB. An important observation from $(6.12)$ is that $F_{\text {system }}$ is dominated by the $F_{\text {LNA }}$ due to high gain of the LNA, meaning the second term on the right hand of (6.12) can be neglected. For the same reason, also the impact of the BRF on the noise figure is neglected. It can be concluded that the $F_{\text {LNA }}$ can be calculated by

$$
F_{\mathrm{LNA} \mid \mathrm{dB}}=S N R_{\text {in } \mid \mathrm{dB}}-S N R_{\text {measured } \mid \mathrm{dB}} .
$$

The calculations were done using $\frac{E_{s}}{N_{0}}$ instead of the SNRs The $E_{s}$ at the input of the LNA is the power of the desired signal divided by the symbol rate. The input noise $N_{0}$ is assumed to be thermal noise with a spectral density of $N_{0}=k T$, where $k$ is the Boltzmann's constant and $T$ is the noise temperature. At room temperature, $T=290$ Kelvin, $N_{0}$ is $-174 \mathrm{dBm} / \mathrm{Hz}$. This is a reasonable assumption for $N_{0}$ with a low output power of the signal generator.

\section{Results}

The blocker noise figure was measured for the desired signal at $394 \mathrm{MHz}$, with an output power of $-90 \mathrm{dBm}$ at the generator. The desired signal is QPSK modulated with 25000 symbols per second. The blocker was set again at $410 \mathrm{MHz}$ and the output power was increased in steps of $1 \mathrm{~dB}$.

The results of the blocker noise figure measurements are presented in Figure 6.7. With no blocker the noise figure of the LNA was measured to be $2.9 \mathrm{~dB}$. The noise figure of the LNA was doubled at a blocker power $P_{b}$ of $-19 \mathrm{dBm}$.

The noise figure model for a blocked RF stage from (6.9) is also included in the graph from Figure 6.7. Up to a $P_{b}$ of $-13 \mathrm{dBm}$, which corresponds to a $F_{\mathrm{LNA}}$ of 11 $\mathrm{dB}$, the model is within $1 \mathrm{~dB}$ of the measured curve. For higher blocker power the model underestimates the increase in noise figure due to the blocker.

An interesting observation from Figure 6.7 is that the measured $F_{\text {LNA }}$ starts to increase from a blocker power of $-27 \mathrm{dBm}$, and that this corresponds well to the start of the gain compression shown in Figure 6.5. 


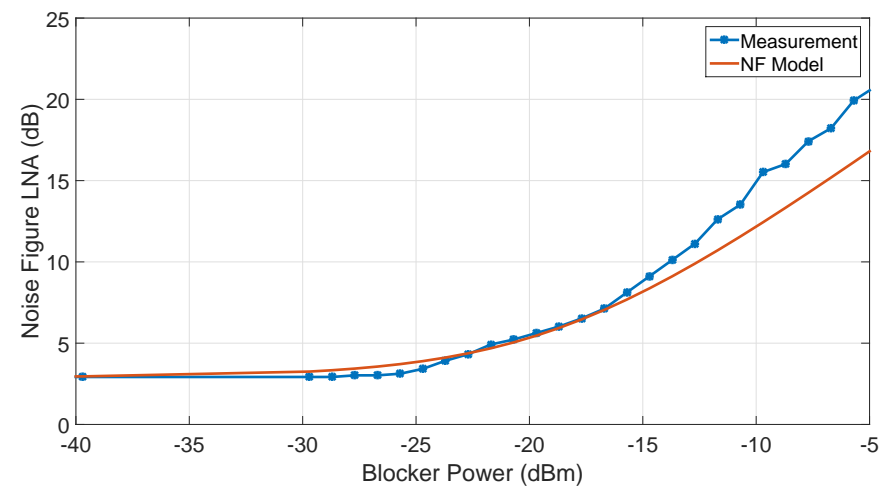

Figure 6.7: $F_{\text {LNA }}$ as a function of blocker power and the noise figure model from (6.9).

\subsection{System performance}

\subsubsection{Modulation formats and bit error probability}

The performance of receivers can be quantified by the bit-error-probability (BEP), which is a function of the $\frac{E_{b}}{N_{0}}$, where $E_{b}$ is the sigal power divided by the bit rate. The $\mathrm{BEP}$ of various modulation methods for an optimum receiver for an AWGN channel are well-studied and resulted in good approximations of the BER [135].

The BEP for three relevant modulation formats, QPSK, $M$-ary phase shift keying ( $M$-PSK) and $M$-ary QAM, are presented in Table 6.1. Binary phase shift keying (BPSK) is a modulation format which has half the bit rate of QPSK, but an equal BEP. The variable $M$ is defined as the number of symbols in the modulation constellation, $k$ is the number of bits per symbol $\left(\log _{2} M\right)$, and the $Q$-function is the tail probability of the normal standard distribution and is given by

$$
Q(x)=\frac{1}{\sqrt{2 \pi}} \int_{x}^{\infty} \exp \left(-\frac{u^{2}}{2}\right) \mathrm{d} u .
$$

The presented BEP assumes that a Gray code is used in the mapping, meaning that a symbol error results in only 1 bit error. The BEPs are good approximations which are valid for $\frac{E_{b}}{N_{0}} \gg 1[135]$.

\subsubsection{BEP curves}

The theory presented in the previous section can be used to estimate the loss in performance of a wireless receiver under various blocker conditions. In Section 6.3.4, the noise figure of a receiving system was measured and it was dominated by the noise figure of the LNA. Of this particular "receiver" we will now show the loss in performance due to desensitization resulting from a blocker. 
Table 6.1: Modulation schemes and bit error probabilities.

\begin{tabular}{|c|c|}
\hline Modulation scheme & Bit error probability \\
\hline \hline BPSK/QPSK & $Q\left(\sqrt{\frac{2 E_{b}}{N_{0}}}\right)$ \\
\hline$M$-PSK & $\frac{2}{k} Q\left(\sqrt{\frac{2 k E_{b}}{N_{0}}} \sin \frac{\pi}{M}\right)$ \\
\hline$M$-QAM & $\frac{4}{k}\left(1-\frac{1}{\sqrt{M}}\right) Q\left(\sqrt{\frac{3 k}{M-1} \frac{E_{b}}{N_{0}}}\right)$ \\
\hline
\end{tabular}

As shown in Table 6.1, the BEP is dependent on the $\frac{E_{b}}{N_{0}}$, and this ratio can be calculated based on the noise figure of the receiving system. The $E_{b} / N_{0}$ at the detector is given by

$$
\left[\frac{E_{b}}{N_{0}}\right]_{\mathrm{dB}}=P_{\mathrm{d} \mid \mathrm{dBm}}-10 \log _{10}(R)-N_{0}-F_{\mathrm{LNA} \mid \mathrm{dB}}\left(P_{b}\right)
$$

where $P_{\mathrm{d} \mid \mathrm{dBm}}$ is the desired signal power in $\mathrm{dBm}, R$ is the data rate in bits per second, $N_{0}$ is $-174 \mathrm{dBm} / \mathrm{Hz}$ and $F_{\mathrm{LNA} \mid \mathrm{dB}}$ is the noise figure of the LNA in dB as shown in Figure 6.7. Obviously, the $F_{\mathrm{LNA}}$ is a function of the blocker power $P_{b}$. For the BEP calculations, the $R$ is set at 500 kilobits per second.

In Figure 6.8, the BEP is plotted as a function of $P_{b}$. The desired signal power $P_{b}$ was set at $-100 \mathrm{dBm}$. The degradation can clearly be seen starting from $-27 \mathrm{dBm}$, that is the point where the noise figure of the receiver started to degrade. It can also be seen that, as expected, QPSK has a better BEP than the other two modulation formats. This comes at the cost of a lower spectral efficiency [65]. For high levels of $P_{b}$ the $\frac{E_{b}}{N_{0}}$ is severely degraded and the performance of all formats are poor and seem to be converging.

The system performance for one individual modulation format, in this case QPSK, is better depicted in Figure 6.9. In this figure, $P_{d}$ is varied in steps of $0.5 \mathrm{~dB}$ from $-115 \mathrm{dBm}$ up to $-90 \mathrm{dBm}$. For every step, the BEP is calculated, and four curves are plotted for 4 different blocker power levels; off, -20, -15, and -10 dBm.

Figure 6.9 shows the system performance depending on the received signal strength. As can be seen, due to the blocker the BEP significantly increases, resulting in a poor sensitivity. Another observation that can be made from Figure 6.9, is that as long as the received signals are well above the sensitivity levels of the receiver, the effect of desensitization is still minimal. For instance, a received signal power of $-90 \mathrm{dBm}$, with a $-10 \mathrm{dBm}$ blocker, shows a BEP of approximately $10^{-8}$, which is still adequate.

However, it is important to realize that the presented BEP curves are assumed for AWGN, and the degradation arises from a degraded noise figure. In practical 


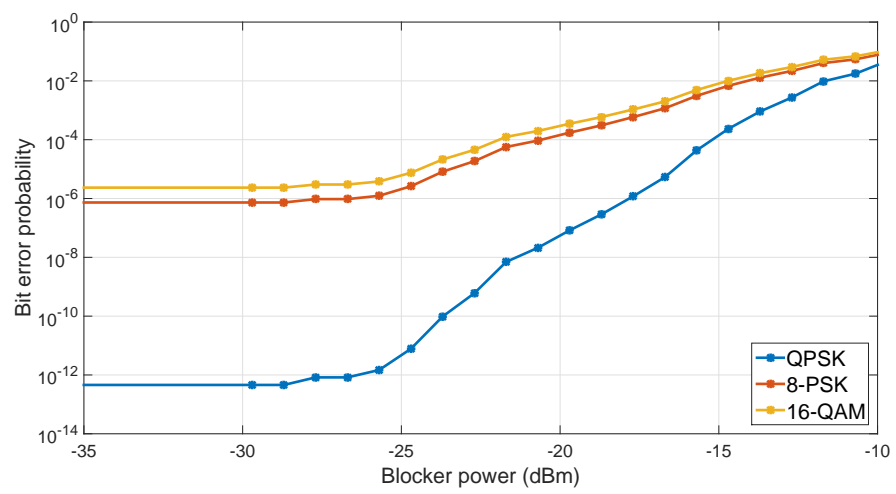

Figure 6.8: BEP as a function of blocker power for a received desired signal of -100 $\mathrm{dBm}$.

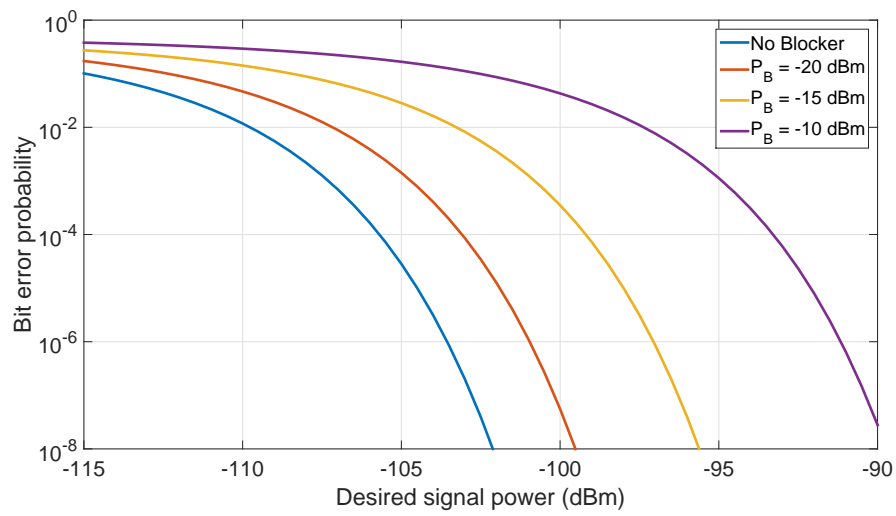

Figure 6.9: BEP as a function of desired signal power for QPSK with various blocker power levels.

situations it is possible that the spurious responses generated by the saturated receiver are in-channel of the desired signal. These spurious responses would degrade the performance much faster.

\subsection{Discussion on possible improvements}

One of the most obvious improvements would be the development of blocker-tolerant receivers for wideband applications. Luckily, there has recently been a great deal of research in the field of solid-state circuits for developing this type of receivers. The reason for these developments are that wideband $\mathrm{RF}$ receivers are considered to be 
necessary to keep up with the increasing number of new frequency bands used for wireless communication. It does not scale to let each new mode of communication have their own radio and baseband chipset on the mobile device [136].

A solution was found in a wideband radio receiver that can be tuned electronically to the band of interest [136], however, this requires to omit the narrowband front end filter. Front end filters are almost always fixed and cannot be tuned, and for this reason, multiple front end filters and a switch would be necessary to cover the many different bands for a wideband receiver. This is not desirable from a functional point of view due to the physical volume this occupies. These developments resulted in the design of blocker-tolerant receivers without front end filters suitable for wideband wireless applications [137-139].

An interesting overview of various blocker-tolerant receiver architectures is presented in [140]. The most promising techniques omit amplification at RF frequencies and employ passive-mixer first receivers $[141,142]$. These receivers avoid voltage gain at $\mathrm{RF}$ frequencies, preventing the blocker from saturating the receiver. The signal is first mixed to lower frequencies with highly linear passive mixers and the filtering and amplification can then be done at IF or baseband. These types of receivers show exceptionally good linearity, but it does come at a cost of a degraded noise performance.

A completely different approach to increase robustness against desensitization is reducing the data rate. From (6.15) it can be seen that $\frac{E_{b}}{N_{0}}$ is dependent on the data rate $R$. In the examples of system performance presented in Section 6.4, we used a data rate of 500 kilobits per second. A reduction of the data rate to 50 kilobits per second, would improve the $\frac{E_{b}}{N_{0}}$ with $10 \mathrm{~dB}$, which is a significant improvement in terms of BEP. For critical applications, such as the public safety communications, the data rate is not the key priority. Reducing the data rate under harsh conditions increases the robustness of the system. Of course, the implementation of error detection and correction improves the robustness against bit errors as well.

\subsection{Summary and conclusions}

Spread spectrum techniques are employed to increase the robustness against jamming attacks, but this does not help against a blocker saturating the receiver. In this chapter, the blocking and desensitization effects of a large interferer on a wireless receiver have been investigated. An experimental method was presented that can be used to measure the blocker noise figure of a DUT.

The blocking mechanisms, non-linear effects and desensitization, were described. These mechanism were confirmed during the characterization of an LNA under blocking conditions. These detrimental effects at the RF stages were next translated to the 
impact it has on the system performance of a wireless receiver for various modulation formats.

Finally, possible improvements for a receiver system were discussed. Recent developments in the field of solid-state circuits resulted in more robust receiver architectures against blockers. Another effective countermeasure against desensitization is decreasing the data rate of the wireless system.

This the end of the susceptibility analysis in this thesis. In Chapter 2, the threat of IEMI to the wireless infrastructure was discussed, Chapter 3 identified the relevant interference mechanisms, and Chapter 4 to Chapter 6 further investigated these interference mechanisms. Along this analysis generic evaluation methods were presented and possible countermeasures were identified. The next step is to develop a methodology to protect critical infrastructures against IEMI, based on the threat defined in Chapter 2. This is the topic of the next chapter. 


\section{Chapter 7}

\section{Protection strategies against IEMI}

In this chapter, a methodology is presented for estimating the required protection levels of critical equipment against IEMI, and evaluating applicable protection techniques. This work is the outcome of joint work within the STRUCTURES project. The results have been presented at the IEEE International Symposium on Electromagnetic Compatibility in 2016 [143].

\subsection{Background on protection strategies}

Throughout this thesis, the vulnerability of wireless communication against IEMI is investigated. In Chapter 3, the three interference mechanisms - damage, saturation, and jamming - were described and existing countermeasures were presented. A summary of the most common protection techniques or systems traits that increase the robustness against these mechanisms are presented in Table 7.1. It is important to note that the protection strategies improve the robustness of wireless communication against IEMI, but it does not make them invulnerable.

Another possibility to protect victim systems, that is general to any electronic system, is to restrict the minimum distance to which an adversary carrying a IEMI source could approach the critical systems. Increasing distance between source and victim diminishes the amplitude of the incoming disturbance. For instance, in [144], the authors discuss spatial retreat for the mobile equipment as a countermeasure against jamming.

Spatial retreat is not possible for fixed systems such as a base station. However, for fixed systems, the minimum distance between attacker and victim can be controlled 
Table 7.1: Interference mechanisms and possible protection strategies.

\begin{tabular}{|l|l|}
\hline & Countermeasures \\
\hline \hline Damage & Limiter, GaN-based front ends, front end filter. \\
\hline Saturation & $\begin{array}{l}\text { Blocker-tolerant receivers, front end filter, coding } \\
\text { schemes, reduced data rate. }\end{array}$ \\
\hline Jamming & $\begin{array}{l}\text { High selectivity, spread spectrum techniques, smart } \\
\text { antennas, coding schemes, scrambling of control } \\
\text { channels. }\end{array}$ \\
\hline
\end{tabular}

by applying access control. Critical systems can be kept at a location that is not accessible for non-authorized people, i.e., at the heart of a building. Another example, is fencing the area around a critical system to increase the minimum distance.

In [34], the vulnerability of the GSM-R standard (GSM standard for railway usage) was studied. The research focused on the expected threat levels due to IEMI, and on the susceptibility levels of the equipment. In this chapter, we extend the work in [34] by providing a systematic analysis to determine the required protection levels. This overall analysis methodology is applicable to any critical infrastructure, i.e., it is not restricted to the wireless communication infrastructure.

Based on the required protection levels, we identify applicable protection techniques for wireless communication. Finally, we investigate the associated costs, both monetary and due to loss of performance, associated with the implementation of the protection techniques. The method to assess the monetary costs can be used for a quantitative comparison of different protection techniques that are suitable for the protection of the infrastructure. We are mainly interested in presenting a method that can be used to compare different techniques and not in the exact assessment of the costs. Again, the method to assess the monetary costs is applicable to any critical infrastructure.

This chapter is organized as follows. The procedure for estimating the protection levels is presented in Section 7.2. The application of the methodology to the case of a base station is shown in Section 7.3. The goal is to protect the wireless receiver of the base station against damage. The possible protection schemes and their selection based on costs are discussed in Sections 7.4 and 7.5, respectively. Finally, conclusions and a summary are given in Section 7.6. 


\subsection{Procedure for estimating the required protec- tion levels}

In this section, we set up a general procedure to assess the required protection levels for a critical infrastructure.

First, one has to collect relevant information regarding the infrastructure:

1. The typical configurations of the topological and interaction sequences diagrams of the infrastructures under study;

2. The typical attenuation levels imposed by the different EM barriers that will be encountered by a travelling wave while it propagates towards the infrastructure;

3. An estimation of the radiated and conducted susceptibility levels of the critical equipment according to what has been reported in the literature and standards.

In Chapter 2.5, a typical configuration of a wireless network is presented and in Chapter 2.3.1, we provided the coupling from the EM waves to a wireless receiver. In Chapter 3, an estimation is given of the susceptibility levels concerning a wireless infrastructure.

Then, one has to implement the following steps:

(i) Create a possible scenario by choosing an IEMI source and the configuration of the EM topology; starting from the source up to the critical equipment (victim).

(ii) Obtain the far voltage - that is, the product of the peak electric field measured in the far field and the range - of the IEMI source and its fundamental frequency.

(iii) Obtain the typical transfer functions of the EM barriers found in the topological diagram.

(iv) Obtain the susceptibility threshold of the equipment.

(v) Calculate the total transfer function by adding the transfer functions of all the successive EM barriers.

(vi) Estimate the total received voltage/field at the equipment level.

(vii) Calculate the difference between the received voltage/field and the susceptibility threshold; and add the required extra protection in order to ensure compatibility.

In the first step of this procedure, the scenario is selected based upon a possible threat (realistic IEMI source) and a realistic source location. A list of potential IEMI sources can be found in [57], where the sources are assessed and characterized based on both technical and non-technical attributes, as discussed in Chapter 2.2. 
Given the source characteristics and its location, the procedure will result in an estimated total received voltage/field at the equipment level. If this exceeds the susceptibility threshold, the protection needed is calculated as the difference between the received signal strength and the susceptibility level. We suggest to add a 10 $\mathrm{dB}$ safety margin to ensure compatibility. The whole procedure can be iterated for different scenarios.

\subsection{Required protection levels for a typical base station}

In this section, we calculate the required protection levels for a typical base station using the previously described procedure. The goal is to protect the wireless receiver against damage. The critical component is the LNA in the front end. We assume that its damage level is $34 \mathrm{dBm}$ (in this case we use a threshold in terms of power rather than voltage), which was estimated in Chapter 3.4.1. As mentioned, this is an estimate and the LNA damage level can vary for different types of LNA.

\subsubsection{Topological decomposition of the IEMI scenario}

The topological diagram of the proposed scenario is presented in Figure 7.1 and is inspired by typical setup of a base station. A dipole antenna located on a tall mast is being used to receive the wireless communication signals. We assume it is at a height $H$ of 10 meters above ground.

The antenna is directly connected to a front end, whose typical structure is shown in Figure 7.2. This structure can vary depending on the manufacturer. The base station, where the actual digital processing is done, is at ground level. The front end and the base station are connected via an RG214 cable. Finally, we assume the base station operates in the 380 to $385 \mathrm{MHz}$ frequency band.

\subsubsection{IEMI source description}

To choose the sources for this case, we have considered transportable devices that could be brought close to the station by a person or by a vehicle. We specify the generated field strengths by the far voltage, which is defined as the product of the peak electric field measured in the far field and the range (distance). In this analysis we study the effect of two different sources assumed to be at a distance $r$ of 20 meters (see Figure 7.1). This implies that the separation between the source and the mast $S$ is $17.3 \mathrm{~m}$.

The first source is a standard T-DIEHL HPEM case [145]. It is a mesoband source and it can be tuned to operate at a frequency of $395 \mathrm{MHz}$ providing a far voltage 


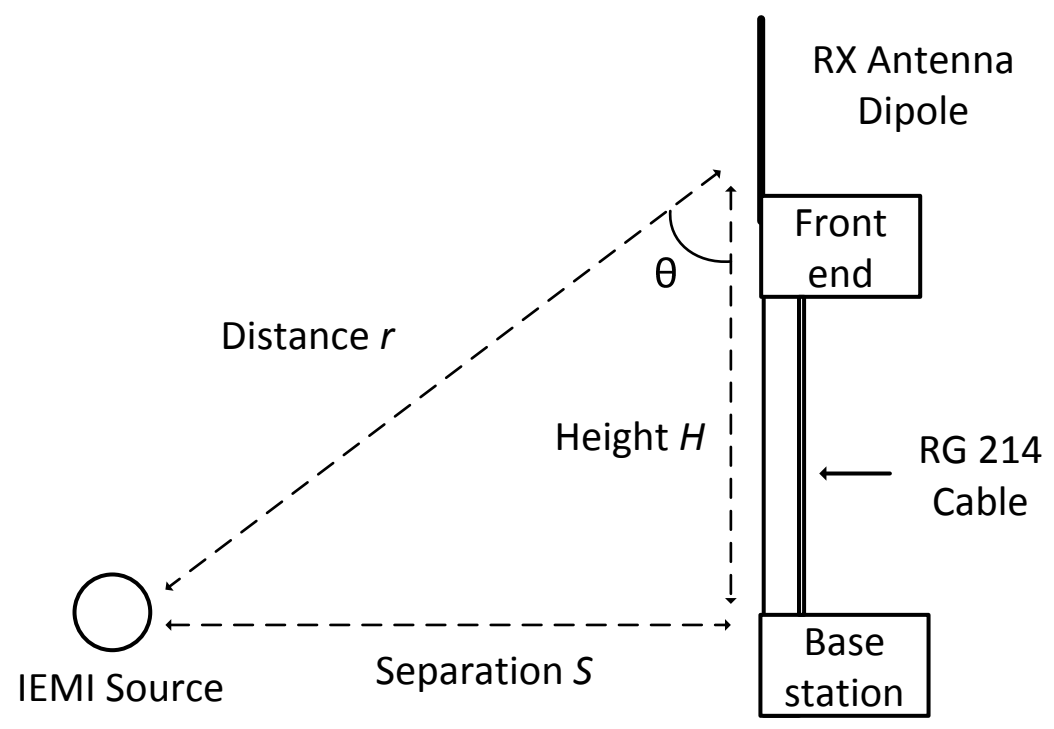

Figure 7.1: EM topological diagram of the front-door illumination of the base station receiver.

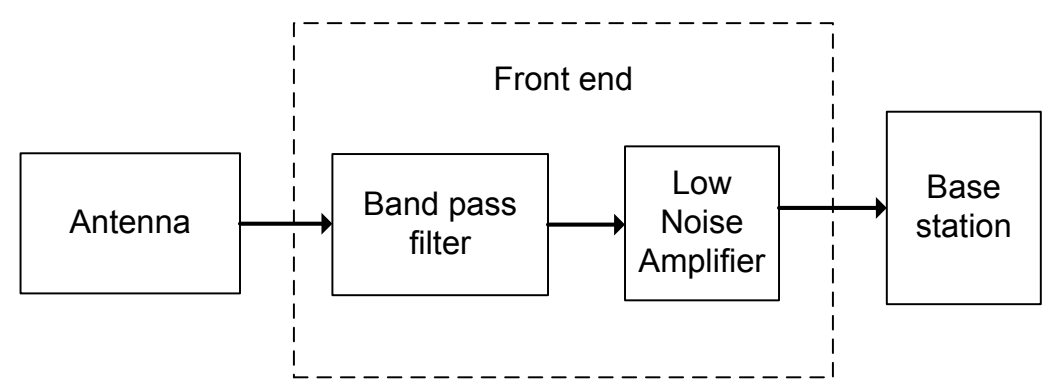

Figure 7.2: Typical structure of the front end at a TETRA base station.

of $225 \mathrm{kV}$. The frequency was purposely chosen as an out-of-band (OOB) frequency. The second source is a switching oscillator connected to a monopole antenna [146]. It is a mesoband source that is tuned at the frequency of $385 \mathrm{MHz}$, in-band, and generates a far voltage of $6.6 \mathrm{kV}$. A summary of the parameters of the considered sources is presented in Table 7.2 .

\subsubsection{Obtaining the required protection level}

At a distance of $20 \mathrm{~m}$, the incident field strength of Source 1 and Source 2 will be respectively $11.25 \mathrm{kV} / \mathrm{m}$ and $0.33 \mathrm{kV} / \mathrm{m}$. The received power is calculated using Eq. $(2.7)$. 
Table 7.2: Parameters of the considered sources

\begin{tabular}{|c|c|c|c|c|}
\hline Source ID & $\begin{array}{c}\text { Source } \\
\text { type }\end{array}$ & Description & $f(\mathbf{M H z})$ & $\begin{array}{c}\text { Far voltage } \\
(\mathbf{k V})\end{array}$ \\
\hline \hline Source 1 & Mesoband & $\begin{array}{c}\text { A standard T } \\
\text { Diehl HPEM case. }\end{array}$ & 395 & 225 \\
\hline Source 2 & Mesoband & $\begin{array}{c}\text { Switching oscillator } \\
+ \text { Monopole } \\
\text { antenna }\end{array}$ & 385 & 6.6 \\
\hline
\end{tabular}

As a receiving antenna we use a half-wave dipole antenna which is commonly used by TETRA networks [89]. It has an $8 \%$ bandwidth from $380 \mathrm{MHz}$ up to $410 \mathrm{MHz}$ with a reflection coefficient $\Gamma$ of $0.316(-10 \mathrm{~dB})$. The gain of an omnidirectional dipole antenna is only dependent on the angle $\theta$, see Figure 7.1 , and is given by [61]:

$$
G(\theta)=1.64\left[\frac{\cos \left(\frac{\pi}{2} \cos \theta\right)}{\sin (\theta)}\right]^{2}
$$

The maximal gain is at $\theta=0$ and is $1.64(2.15 \mathrm{dBi})$. Furthermore, we set $Z_{0}$ at $377 \Omega$ and we assume the polarization mismatch $e_{\mathrm{p}}$ between the transmitter and the receiver to be 0.75 .

The received power is attenuated by the BPF before the LNA. A typical filter characteristic of a band pass filter was presented in Figure 3.3. It shows an OOB attenuation of over $80 \mathrm{~dB}$, and an in-band insertion loss of $1 \mathrm{~dB}$. This means that Source 1 is attenuated by $80 \mathrm{~dB}$ and Source 2 only experiences a $1 \mathrm{~dB}$ insertion loss. As a result, the approximate input power at the LNA due to Source 1 and Source 2 is, respectively, $-9 \mathrm{dBm}$ and $39 \mathrm{dBm}$. The front end filter is successful in attenuating the OOB interference from Source 1 below the damage levels. However, the in-band interference of Source 2 exceeds the threshold by $5 \mathrm{~dB}$. The results are summarized in Table 7.3.

It is important to understand that we purposely chose Source 1 to be OOB. If it was in-band, the received power would exceed the damage level of the LNA by $35 \mathrm{~dB}$, so we can conclude that the front end filter is very effective in mitigating the impact of OOB interference.

\subsection{Identification of protection techniques}

There are few methods available for the protection of front-door coupling since the additional measures taken to attenuate the incoming interferences will also contribute 
Table 7.3: Required protection levels.

\begin{tabular}{|c|c|c|c|c|}
\hline Source ID & $\begin{array}{c}\text { Incident } \\
\text { field } \\
\text { strength } E \\
(\mathbf{k V} / \mathbf{m})\end{array}$ & $\begin{array}{c}\text { Filter at- } \\
\text { tenuation } \\
(\mathbf{d B})\end{array}$ & $\begin{array}{c}\text { Power at } \\
\text { the LNA } \\
(\mathbf{d B m})\end{array}$ & $\begin{array}{c}\text { Required } \\
\text { Protection } \\
(\mathbf{d B})\end{array}$ \\
\hline \hline Source 1 & 11.25 & 80 & -9 & $\mathrm{~N} / \mathrm{A}$ \\
\hline Source 2 & 0.33 & 1 & 39 & 5 \\
\hline
\end{tabular}

to degrading the operation of the system in normal conditions. Therefore, the chosen methods need to provide a very low insertion loss.

\subsubsection{Fencing}

A possible way to diminish the amplitude of the incoming disturbance is to restrict the minimum distance to which a source could approach the communication tower. The minimum distance can be restricted with the use of a protection fence at a given radius from the tower, so as to increase the separation distance $S$.

The additional attenuation compared to $r=20 \mathrm{~m}$ as a function of the radius can be estimated with the following equation:

$$
A t t=20 \log _{10}\left(\frac{\sqrt{S^{2}+H^{2}}}{20}\right)
$$

where $S$ is the separation and $H$ the height of the base station as indicated in Figure 7.1. The additional attenuation by installing a fence at a $S$ of $35 \mathrm{~m}$ is $5.2 \mathrm{~dB}$. However, if we would like to add a $10 \mathrm{~dB}$ safety margin, the fence would have to be at a distance of $112 \mathrm{~m}$.

In the previous derivation, we even neglected the fact that with increasing $S$, the IEMI gets closer to the boresight of receiving dipole, i.e. the gain of the dipole antenna increases (see (7.1)). If this is taken into account, the distance of the fence should be further increased. However, base station towers are often situated at populated areas and therefore it is not easy to put a fence with a large radius around a base station.

This all shows that a fence is not a practical solution to protect the base station and is not further taken into account as a protection strategy.

\subsubsection{RF limiter}

Perhaps the only effective (commercially available) measure against front-door attacks of LNAs is the use of RF limiters based on PIN diodes. Several vendors provide discrete pin diodes that can be used to limit the incoming power to an LNA. Typical 
limitation levels are about $20 \mathrm{dBm}$ for input powers between $1 \mathrm{~W}-1000 \mathrm{~W}$. The insertion loss of these devices can be typically between 0 to $3.5 \mathrm{~dB}$ which is a very good rating for the front-door protection.

For the application, the required pin diodes usually work in the band between 50 $\mathrm{MHz}$ and $4 \mathrm{GHz}$. Since the considered sources are pulsed sources, the CW power that the pin diodes should tolerate is low. Notice that the installation of an RF limiter will provide protection to the LNA from damage but will impede the operation of the system during an attack since communication is not possible if the limiter is active.

\subsection{Evaluation of the protection technique}

\subsubsection{Monetary costs}

To assess the monetary cost of implementing a protection scheme, we divide the total life-cycle expenditures into three main categories: initial cost, yearly operational cost, and yearly maintenance cost. The initial cost greatly depends on whether the required equipment and components are available for purchase or if they will need to be designed and manufactured. It also depends on the effort required for the installation of the protection scheme. A yearly operational cost exists if a protection scheme requires the presence of an operator to run it, or if it necessitates an energy supply or any other resources needed for its uninterrupted operation. A yearly maintenance cost is determined by the hardware and/or software maintenance costs, and the cost of yearly checks, if applicable.

We propose here the following formulas for calculating the mentioned categories of the protection cost:

$$
\begin{aligned}
C_{\mathrm{INIT}}= & P+\left\{H_{\mathrm{HW}} \cdot P P H_{\mathrm{HW}}+H_{\mathrm{SW}} \cdot P P H_{\mathrm{SW}}\right. \\
& \left.+C_{\mathrm{PU}} \cdot U\right\}+H_{\mathrm{IN}} \cdot P P H_{\mathrm{IN}} \\
C_{\mathrm{YM}}= & H_{\mathrm{HW}, \mathrm{YM}} \cdot P P H_{\mathrm{HW}}+H_{\mathrm{SW}, \mathrm{YM}} \cdot P P H_{\mathrm{SW}} \\
& +C_{\mathrm{YT}}
\end{aligned}
$$

The cumulative cost after $N_{\mathrm{Y}}$ years of use is given by:

$$
C=C_{\mathrm{INIT}}+\left(C_{\mathrm{YOP}}+C_{\mathrm{YM}}\right) \cdot N_{\mathrm{Y}}
$$

The variables in Eq. (7.3) to (7.5) are defined in Table 7.4.

The costs of installing an RF limiter equals the initial costs, as there are no yearly operational or yearly maintenance costs. The hourly rates to be used in (7.3) depend not only on the type of work but also on the particular country where the work is 
Table 7.4: Elements participating in the cost of implementing a protection method.

\begin{tabular}{|c|c|}
\hline Cost & Description \\
\hline$C_{\mathrm{INIT}}$ & Initial cost \\
\hline$P$ & $\begin{array}{l}\text { Price if protection elements are available for purchase (price per } \\
\text { element times the number of protection elements). }\end{array}$ \\
\hline$H_{\mathrm{HW}}$ & Hours of HW design $(\geq 0)$. \\
\hline$P P H_{\mathrm{HW}}$ & Price-per-hour of HW design. \\
\hline$H_{\mathrm{SW}}$ & Hours of SW design $(\geq 0)$ \\
\hline$P P H_{\mathrm{SW}}$ & Price-per-hour of SW design. \\
\hline$C_{\mathrm{PU}}$ & $\begin{array}{l}\text { Cost of material per unit. One unit can be a surface unit, length } \\
\text { unit, an element, etc. }\end{array}$ \\
\hline$U$ & Number of units $(\geq 0)$. \\
\hline$H_{\mathrm{IN}}$ & Hours of installation. \\
\hline$P P H_{\mathrm{IN}}$ & Price-per-hour of installation. \\
\hline$C_{\mathrm{YOP}}$ & Yearly operational cost (handling, power consumption, etc.). \\
\hline$C_{\mathrm{YM}}$ & Yearly maintenance cost. \\
\hline$H_{\mathrm{HW}, \mathrm{YM}}$ & Hours of HW maintenance $(\geq 0)$ \\
\hline$P P H_{H W, M}$ & Price-per-hour of HW maintenance. \\
\hline$H_{\mathrm{SW}, \mathrm{YM}}$ & Hours of SW maintenance $(\geq 0)$. \\
\hline$P P H_{\mathrm{SW}, \mathrm{M}}$ & Price-per-hour of SW maintenance. \\
\hline$C_{\mathrm{YT}}$ & Cost per yearly testing. \\
\hline
\end{tabular}


Table 7.5: Estimation of the costs of implementing RF limiter protection scheme.

\begin{tabular}{|l|c|c|c|c|c|c|}
\hline Description & $P(€)$ & $H_{\mathrm{HW}}$ & $\begin{array}{c}C_{\mathrm{PU}} \\
(€)\end{array}$ & $U$ & $H_{\mathrm{IN}}$ & $\begin{array}{c}C_{\mathrm{INIT}} \\
(€)\end{array}$ \\
\hline $\begin{array}{l}\text { RF limiter max. CW } \\
\text { power 1000W }\end{array}$ & 2000 & 20 & 500 & 1 & 20 & 5500 \\
\hline $\begin{array}{l}\text { RF limiter max. CW } \\
\text { power 100W }\end{array}$ & 200 & 20 & 500 & 1 & 20 & 3700 \\
\hline $\begin{array}{l}\text { RF limiter max. CW } \\
\text { power 10W }\end{array}$ & 100 & 20 & 500 & 1 & 20 & 3600 \\
\hline
\end{tabular}

carried out. For simplicity, and since it is not our goal here to calculate the required budget exactly, we will assume the following rates in Euros: $P P H_{\mathrm{HW}}=100$ and $P P H_{\mathrm{IN}}=50$.

An approximate estimate of the costs of installing a limiter is presented in Table 7.5. The costs presented in Table 7.5 are an educated guess based on experience and discussions with experts in this field. The price of adding an RF limiter to a base station is primarily influenced by the costs of material and personnel. However, these costs are fixed, whereas the price of the RF limiter itself depends on the power it should withstand: the higher the power, the higher the price.

\subsubsection{Loss in performance}

Another cost of using an RF limiter as a protection scheme is the loss in performance. Let us investigate the receiver noise factor $F$ (or noise figure when expressed in decibels) in the presence of an RF limiter.

Figure 7.3 shows a typical receiver configuration. The total noise factor, $F_{\text {total }}$, depends on the noise factor of individual components and on their respective gains, and can be estimated using the Friis' formula:

$$
F_{\text {total }}=F_{1}+\frac{F_{2}-1}{G_{1}}+\frac{F_{3}-1}{G_{1} G_{2}}+\frac{F_{4}-1}{G_{1} G_{2} G_{3}}
$$

Typical values of the gain and the noise factor are given in Table 7.6. For the passive components, such as the filter, the limiter, and the cable, the noise factor equals the insertion loss of the component itself [147].

Without the limiter, $F_{\text {total }}$ is $3 \mathrm{~dB}$. Installing a limiter with an insertion loss $L$ of 1 $\mathrm{dB}$ will degrade $F_{\text {total }}$ by $1 \mathrm{~dB}$. This results in $1 \mathrm{~dB}$ loss of sensitivity. From (7.6), we can see that $F_{\text {total }}$ depends linearly on the noise factor of the limiter, as $F_{2}=L_{2}$ and $G_{2}=1 / L_{2}$. Every additional $\mathrm{dB}$ of insertion loss before the LNA is directly added to $F_{\text {total }}$. This is one of the reasons why limiters are not often present in a receivers. 


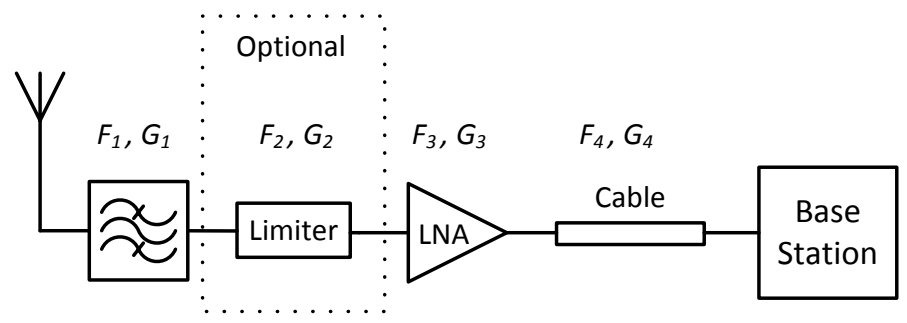

Figure 7.3: Receiver configuration

Table 7.6: Typical values for the gain and the noise figure of receiver components.

\begin{tabular}{|l|c|c|}
\hline Component & Noise Figure (dB) & Gain (dB) \\
\hline \hline 1. Filter & 1 & -1 \\
\hline 2. Limiter & 1 & -1 \\
\hline 3. LNA & 2 & 20 \\
\hline 4. Cable & 4 & -4 \\
\hline
\end{tabular}

\subsection{Summary and conclusions}

In this chapter, the susceptibility of a wireless infrastructure is analyzed and a methodology to estimate the required protection levels is presented. The focus in this chapter was mainly on a communication infrastructure, but the method can be applied to any critical infrastructure.

We showed that available sources at a distance of $20 \mathrm{~m}$ can easily generate an input power at the LNA that exceeds the damage levels of the LNA. The best protection against OOB interference is a front end filter, which significantly reduces the vulnerability. However, wireless receivers showed to be highly vulnerable against in-band interference.

A possible protection technique is the installation of an RF limiter in the front end of the receiver. The possibility of restricting the minimum distance to which a source could approach the communication tower showed not to be effective. IEMI can effectively be coupled into the antenna at a large distance.

A method to estimate the costs of a protection technique is presented. This method is applied on the installation of an RF limiter in a wireless receiver. It showed that the price of adding an RF limiter to a base station is primarily influenced by the costs of material and personnel. The costs presented here are intended only to illustrate the methodology. The actual costs for any particular implementation will depend greatly on the detailed specification, quantities to be installed and whether the protection is 
installed as the infrastructure is built, or fitted to an existing installation.

Finally, we showed that the loss in performance of a wireless receiver is due to an increase in the noise factor. It is essential to keep the insertion loss of the limiter as low as possible to mitigate the loss in performance of the wireless receiver. 


\section{Chapter 8}

\section{Conclusions}

This chapter will summarize the main results and the conclusions that can be drawn from the work presented in this thesis.

\subsection{Summary and conclusions}

In Chapter 1, a background on the threat of IEMI to critical infrastructures was presented. It is shown how the increasing dependency of society upon CIs controlled by electronic systems, together with the technological advances in the development of HPEM sources, lead to the possible vulnerability of society to IEMI. Furthermore, an introduction was presented of wireless communication and the dependence of nowadays society upon wireless networks was explained.

Chapter 2 presented an overview of the threat related to an IEMI attack, which involves both technical and non-technical attributes. A risk analysis involves the IEMI source, coupling path, and the CI. The analysis of an CI starts with investigating the susceptibility by identifying the critical subsystems and their susceptibility levels. Once the levels are known, a risk estimate can be made by combining knowledge on available IEMI sources, possible coupling paths, and the accessibility of the infrastructure. The conclusion is that IEMI poses a serious threat to wireless networks, because of the high vulnerability of wireless communication systems to EMI, and the high likelihood of an jamming attack. This conclusion is supported by numerous accounts of IEMI attacks against various communication systems.

Chapter 3 identified three different interference mechanisms - physical damage, saturation, and jamming - that could result in a DoS of the wireless communication system. The mechanisms are different in nature and therefore require separate protection strategies, which involves various disciplines of expertise. The susceptibility of a TETRA base station was experimentally investigated. Generic experimental meth- 
ods were presented that can be used for susceptibility analysis. The findings in this chapter have been published in the IEEE Transactions on EMC.

In Chapter 4, a general overview of various jamming attacks is presented. The jamming attacks can vary from barrage jamming to highly sophisticated attacks requiring high knowledge of the victim system. The goal of countermeasures against jamming attacks - such as the scrambling of the control channels - is to force the adversary to deploy barrage jamming, which is not power effective and easily detected. Next, the protocol of TETRA is studied to identify possible weaknesses. From the analysis, it is concluded that TETRA can be disrupted by an intelligent jammer. The slotted ALOHA protocol can be interfered by corrupting each access assignment channel block, since the TETRA protocol states that the mobile station will wait indefinitely before transmitting until the access assignment channel can be decoded. This jamming attack is only effective if the victim mobile device wants to set-up a connection. If the base station contacts the mobile then it still can set-up a link since it will reserve slots for the mobile to send its data. These findings were published in the IEEE Transactions on EMC.

Chapter 5 presents the results of a study on the vulnerability of RKE systems against jamming attacks. Even though this system is highly secured by use of encryption and code algorithms, a hacking technique described in literature renders these security measures useless. This technique relies on a jamming attack where the wireless link from the key fob to the receiver is jammed, while the attacker is still able to receive the signal from the key fob. This technique is possible due to the poor performance of the RKE receiver. Low-cost systems equipped with low-priced receivers show a poor selectivity, which makes them vulnerable. Better systems are equipped with heterodyne receivers, which can have much better performance in terms of selectivity. However, the detectors used in these receivers are based on envelope detection. We showed that envelope detectors are highly vulnerable to pulsed interference. An improved RKE system would use a highly selective receiver with a synchronous detector. Pulsed noise interference is not more effective in jamming synchronous detectors as compared to continuous noise interference. RKE systems with synchronous receivers would be less vulnerable against specific hacking attacks. These results were published in the IEEE Transactions on EMC.

In Chapter 6, the blocking and desensitization effects of an high-power interferer on the performance of a receiver are investigated. Spread spectrum techniques are employed to increase the robustness against jamming attacks, but this does not help against a blocker saturating the receiver. A experimental method is presented that can be used to measure the effects of a blocker on a receivers front end. This method is used to characterize a COTS LNA. Next, it is shown how the detrimental effects at the RF stages due to a blocker can be translated to the effect it has on the system performance. The bit-error-probability of various modulations is shown for different 
levels of blocker power and desired signal power allowing system performance comparison. Finally, possible improvements for a receiver system are discussed. Recent developments in the field of solid-state circuits resulted in more robust receiver architectures against blockers. Another effective countermeasure against desensitization is decreasing the data rate of the wireless system.

Chapter 7 presents a generic methodology that can be used to estimate the required protection levels for a CI. Furthermore, it presents a method to analyze the cost of implementing a protection technique. This is useful for deciding what protection technique to implement. The methods were applied to a communication infrastructure, where a realistic scenario of an IEMI attack was presented. It is shown that available sources at a distance of $20 \mathrm{~m}$ can generate input powers at the LNA that exceeds the damage levels. A possible protection technique is the installation of an $\mathrm{RF}$ limiter in the front end of the receiver. The possibility of restricting the minimum distance to which a source could approach the communication tower showed not to be effective. The price of adding an RF limiter to a base station is primarily influenced by the costs of material and personnel. The actual costs for any particular implementation will depend greatly on the detailed specification, quantities to be installed and whether the protection is installed as the infrastructure is built, or fitted to an existing installation.

\section{Overall conclusion}

In this thesis, a detailed risk analysis of IEMI against wireless communication is presented and various reasons are identified why IEMI is a serious threat for wireless communication. A complete vulnerability analysis of wireless communication is presented and the susceptibility levels are thoroughly analyzed. Along this analysis, generic experimental methods are presented which enable a detailed susceptibility analysis of wireless systems. For the different interference mechanisms, various protection techniques and strategies were identified.

It is concluded that the research goals, as stated in Section 1.4, are met. This thesis provides a detailed insight into the vulnerability of wireless communication systems and can be used to improve the robustness of wireless communication against IEMI.

\subsection{Recommendations}

It is expected that the dependency upon wireless communication will further increase in the near future. Concepts such as smart cities, Internet of Things, Machine-toMachine communication are currently being developed and shows to be promising. These trends are supported by the development of new communication protocols, such as $5 \mathrm{G}$, which ever aim at increasing performances in terms of increasing capacity and 
lowering latencies. Often security and vulnerability are not the main priorities when these new systems are developed, i.e. it is being developed from a functional point of view.

As is shown in this thesis, the wireless communication can be severely degraded by various EMI scenarios, which results in a vulnerability of a society dependent upon these systems. For this reason, critical communication systems should be developed with an emphasis on robustness.

As explained, developing and implementing protection strategies for wireless communication spans several disciplines. For instance, a team that aims at developing a robust communication system would consist of

- EMC experts for the characterisation of interference, and the analysis and support towards other disciplines;

- Radio engineers for improvements at the physical layer of communication;

- Antenna engineers for developing smart antennas suppressing interferers;

- Microwave engineers or solid-state circuit designers for developing damage protected and blocker-tolerant front ends.

Close collaboration between these various experts is necessary. For instance, the suppression of interferers by smart antennas is effective, but only after the beam forming has been performed, which is typically after the front ends of the individual $\mathrm{RF}$ receivers. Saturation of the RF receivers cannot be prevented by smart antennas.

Currently, new communication technologies - such as 5G-are being developed and it would be highly recommended to assemble a team of various experts, that play a significant role in the development of new technologies ensuring robust communication systems. To this end, we wrote a COST Action proposal with several European partners to support collaboration between various experts from various European research groups. The aim of the COST Action is to develop guidelines and recommendations for robust communication systems and to provide these guidelines to the relevant standardization bodies. 


\section{References}

[1] "Council Directive 2008/114/EC on the identification and designation of European critical infrastructures and the assessment of the need to improve their protection, 2008 O.J. L 345/75."

[2] J. Moteff and P. Parfomak, "CRS Report for Congress - Critical Infrastructure and Key Assets : Definition and Identification," Congressional Research Service, Tech. Rep., 2004.

[3] S. Rinaldi, J. Peerenboom, and T. Kelly, "Identifying, understanding, and analyzing critical infrastructure interdependencies," Control Systems, IEEE, vol. 21, no. 6 , pp. $11-25$, dec 2001.

[4] "URSI resolution on criminal activities using electromagnetic tools," Minutes of the XXV General Assembly of URSI, 1999.

[5] Electromagnetic compatibility (EMC) Part 2-13: Environment High power electromagnetic (HPEM) environments radiated and conducted, IEC Standards 61000-2-13, 2005.

[6] G. Lugrin, N. Mora, S. Sliman, F. Rachidi, M. Rubinstein, and R. Cherkaoui, "Overview of IEMI conducted and radiated sources: Characteristics and trends," in Electromagnetic Compatibility (EMC EUROPE), 2013 International Symposium on, Sept 2013, pp. 24-28.

[7] H. Ott, Electromagnetic Compatibility Engineering. John Wiley \& Sons, 2009.

[8] C. R. Paul, Introduction to electromagnetic compatibility. Hoboken, NJ: John Wiley \& Sons, 2006.

[9] Protection against lightning Part 1: General principles, IEC Standards 623051, 2013.

[10] Protection against lightning Part 2: Risk management, IEC Standards 62305-2, 2013. 
[11] Protection against lightning Part 3: Physical damages to structures and life hazard., IEC Standards 62305-3, 2013.

[12] Protection against lightning Part 4: Electrical and electronic systems within structures, IEC Standards 62305-4, 2013.

[13] Electromagnetic compatibility (EMC) Part 1-3: General The effects of highaltitude EMP (HEMP) on civil equipment and systems, IEC Standards 61000$1-5,2002$.

[14] Electromagnetic compatibility (EMC) Part 2-9: Description of HEMP environment radiated disturbance, IEC Standards 61000-2-9, 1996.

[15] Electromagnetic compatibility (EMC) Part 2-10: Descritpion of HEMP environment Conducted disturbance, IEC Standards 61000-2-10, 1998.

[16] Series K: Protection against interference: High altitude electromagnetic pulse immunity guide for telecommunication centres, ITU-T K.78, 2009.

[17] W. A. Radasky, C. E. Baum, and M. W. Wik, "Introduction to the special issue on high-power electromagnetics (HPEM) and intentional electromagnetic interference (IEMI)," Electromagnetic Compatibility, IEEE Transactions on, vol. 46, no. 3, pp. 314-321, 2004.

[18] J. Benford, J. Swegle, and E. Schamiloglu, High Power Microwaves, Second Edition. Boca Raton, FL: Taylor \& Francis, 2007.

[19] Electromagnetic compatibility (EMC) Part 1-5: General High power electromagnetic (HPEM) effects on civil system, IEC TR 61000-1-5, 2004.

[20] M. Ianoz, "A comparison between HEMP and HPEM parameters. Effects and mitigation methods," in Electromagnetic Compatibility and 19th International Zurich Symposium on Electromagnetic Compatibility, 2008. APEMC 2008. Asia-Pacific Symposium on, May 2008, pp. 275-278.

[21] C. E. Baum, "Maximization of electromagnetic response at a distance," Electromagnetic Compatibility, IEEE Transactions on, vol. 34, no. 3, pp. 148-153, 1992.

[22] F. Sabath, "Classification of electromagnetic effects at system level," in Electromagnetic Compatibility - EMC Europe, 2008 International Symposium on, 2008, pp. 1-5.

[23] T. E. Humphreys, B. M. Ledvina, M. L. Psiaki, B. W. O'Hanlon, and P. M. Kintner, "Assessing the Spoofing Threat: Development of a Portable GPS Civilian 
Spoofer," Proceedings of the 21st International Technical Meeting of the Satellite Division of The Institute of Navigation (ION GNSS 2008), 2008.

[24] R. Hoad, N. J. Carter, D. Herke, and S. P. Watkins, "Trends in EM susceptibility of IT equipment," Electromagnetic Compatibility, IEEE Transactions on, vol. 46, no. 3, pp. 390-395, 2004.

[25] F. Brauer, F. Sabath, and J. L. ter Haseborg, "Susceptibility of IT network systems to interferences by HPEM," in Electromagnetic Compatibility, 2009. EMC 2009. IEEE International Symposium on, 2009, pp. 237-242.

[26] L. Palisek and L. Suchy, "High Power Microwave effects on computer networks," in EMC Europe 2011 York, 2011, pp. 18-21.

[27] D. Mansson, T. Nilsson, R. Thottappillil, and M. Backstrom, "Propagation of UWB Transients in Low-Voltage Installation Power Cables," Electromagnetic Compatibility, IEEE Transactions on, vol. 49, no. 3, pp. 585-592, 2007.

[28] D. Mansson, R. Thottappillil, and M. Backstrom, "Propagation of UWB Transients in Low-Voltage Power Installation Networks," Electromagnetic Compatibility, IEEE Transactions on, vol. 50, no. 3, pp. 619-629, 2008.

[29] J. H. Hagmann, S. Dickmann, and S. Potthast, "Application and propagation of transient pulses on power supply networks," in EMC Europe 2011 York, 2011, pp. $7-12$.

[30] D. Nitsch, M. Camp, F. Sabath, J. L. ter Haseborg, and H. Garbe, "Susceptibility of some electronic equipment to HPEM threats," Electromagnetic Compatibility, IEEE Transactions on, vol. 46, no. 3, pp. 380-389, 2004.

[31] M. Backstrom and K. Lovstrand, "Susceptibility of electronic systems to highpower microwaves: summary of test experience," Electromagnetic Compatibility, IEEE Transactions on, vol. 46, no. 3, pp. 396-403, Aug 2004.

[32] Y. V. Parfenov, L. N. Zdoukhov, W. A. Radasky, and M. Ianoz, "Conducted IEMI threats for commercial buildings," Electromagnetic Compatibility, IEEE Transactions on, vol. 46, no. 3, pp. 404-411, 2004.

[33] D. Mansson, R. Thottappillil, T. Nilsson, O. Lunden, and M. Backstrom, "Susceptibility of Civilian GPS Receivers to Electromagnetic Radiation," Electromagnetic Compatibility, IEEE Transactions on, vol. 50, no. 2, pp. 434-437, 2008 . 
[34] D. Mansson, R. Thottappillil, M. Backstrom, and O. Lunden, "Vulnerability of European Rail Traffic Management System to Radiated Intentional EMI," Electromagnetic Compatibility, IEEE Transactions on, vol. 50, no. 1, pp. 101109, 2008.

[35] M. Camp, H. Gerth, H. Garbe, and H. Haase, "Predicting the breakdown behavior of microcontrollers under EMP/UWB impact using a statistical analysis," Electromagnetic Compatibility, IEEE Transactions on, vol. 46, no. 3, pp. 368-379, Aug 2004.

[36] Electromagnetic compatibility (EMC) Part 4-33: Testing and measurement techniques Measurement methods for high-power transient parameters, IEC Standards 61000-4-33, 2005.

[37] Electromagnetic compatibility (EMC) Part 4-35: Testing and measurement techniques - HPEM simulator compendium, IEC TR 61000-4-35, 2009.

[38] Electromagnetic compatibility (EMC) Part 4 - 36: Testing and measurement techniques IEMI Immunity Test Methods for Equipment and Systems, IEC Standards 61000-4-36, 2013.

[39] Electromagnetic compatibility (EMC) Part 5-9: Installation and mitigation guidelines System-level susceptibility assessments for HEMP and HPEM, IEC Standards 61000-5-9, 2009.

[40] STRUCTURES website. [Online]. http://www.structures-project.eu/.

[41] HIPOW website. [Online]. http://www.hipow-project.eu/hipow/.

[42] SECRET website. [Online]. http://www.secret-project.eu/.

[43] S. van de Beek and F. Leferink, "Current intentional EMI studies in Europe with a focus on STRUCTURES," in Electromagnetic Compatibility, Tokyo (EMC'14/Tokyo), 2014 International Symposium on, May 2014, pp. 402-405.

[44] S. van de Beek, J. Dawson, I. Flintoft, F. Leferink, N. Mora, F. Rachidi, and M. Righero, "Overview of the European project STRUCTURES," IEEE Electromagnetic Compatibility Magazine, vol. 3, no. 4, pp. 70-79, th 2014.

[45] S. van de Beek, J. Dawson, L. Dawson, I. Flintoft, H. Garbe, F. Leferink, B. Menssen, N. Mora, F. Rachidi, M. Righero, M. Rubinstein, and M. Stojilović, "The european project STRUCTURES: Challenges and results," in 2015 IEEE International Symposium on Electromagnetic Compatibility (EMC), Aug 2015, pp. 1095-1100. 
[46] NATO, "Technology Trends Survey. Future Emerging Technology Trends." February 2015.

[47] Ericsson, "Ericsson Mobility Report - On the Pulse of the Network Society," November 2015.

[48] D. Pozar, Microwave Engineering. John Wiley \& Sons, 2005.

[49] Jammer from China. [Online]. Available: http://www.jammerfromchina.com.

[50] POLDERCRASH 25 februari 2009 - Een onderzoek door de Inspectie Openbare Orde en Veiligheid, in samenwerking met de Inspectie voor de Gezondheidszorg, Report issued by the Ministry of the Interior and Kingdom Relations, 2009.

[51] B. Menssen, M. Mleczko, H. Garbe, K. U. Rathjen, S. Dickmann, S. van de Beek, and F. Leferink, "Reference configurations for the characterization of critical infrastructures," in 2014 International Symposium on Electromagnetic Compatibility, Sept 2014, pp. 1218-1223.

[52] E. Genender, H. Garbe, and F. Sabath, "Probabilistic risk analysis technique of intentional electromagnetic interference at system level," IEEE Transactions on Electromagnetic Compatibility, vol. 56, no. 1, pp. 200-207, Feb 2014.

[53] D. V. Giri and F. M. Tesche, "Classification of intentional electromagnetic environments (IEME)," Electromagnetic Compatibility, IEEE Transactions on, vol. 46, no. 3, pp. 322-328, 2004.

[54] F. Sabath, M. Backstrom, B. Nordstrom, D. Serafin, A. Kaiser, B. A. Kerr, and D. Nitsch, "Overview of four European high-power microwave narrow-band test facilities," Electromagnetic Compatibility, IEEE Transactions on, vol. 46, no. 3, pp. 329-334, 2004.

[55] W. D. Prather, C. E. Baum, R. J. Torres, F. Sabath, and D. Nitsch, "Survey of worldwide high-power wideband capabilities," Electromagnetic Compatibility, IEEE Transactions on, vol. 46, no. 3, pp. 335-344, 2004.

[56] D. Mansson, "Intentional Electromagnetic Interference (IEMI). Susceptibility investigations and classification of civilian systems and equipment." Ph.D. dissertation, Uppsala Universitet, 2008.

[57] N. Mora, F. Vega, G. Lugrin, F. Rachidi, and M. Rubinstein, "Study and Classification of Potential IEMI Sources," System Design and Assessment Notes, Note 41, 2014. 
[58] F. Sabath and H. Garbe, "Risk potential of radiated HPEM environments," in Electromagnetic Compatibility, 2009. EMC 2009. IEEE International Symposium on, 2009, pp. 226-231.

[59] Series K: Protection against Interference. High-power electromagnetic immunity guide for telecommunication systems, ITU-T K.81, 2009.

[60] D. Mansson, M. Backstrom, and R. Thottappillil, "Intentional EMI against critical infrastructures, a discussion on mitigation philosophy," in 2010 AsiaPacific International Symposium on Electromagnetic Compatibility, April 2010, pp. $134-137$.

[61] C. Balanis, Antenna Theory, 2nd ed. New York: John Wiley \& Sons, 1997.

[62] D. Mansson, R. Thottappillil, and M. Backstrom, "Methodology for Classifying Facilities With Respect to Intentional EMI," Electromagnetic Compatibility, IEEE Transactions on, vol. 51, no. 1, pp. 46-52, feb. 2009.

[63] J. P. Parmantier, "Numerical coupling models for complex systems and results," IEEE Transactions on Electromagnetic Compatibility, vol. 46, no. 3, pp. 359367, Aug 2004.

[64] Department of Defense Standard Practice - System Safety, MIL-STD-882E, 2012 .

[65] A. Molisch, Wireless Communications. John Wiley \& Sons Ltd., 2011.

[66] M. Stahlberg, "Radio Jamming Attacks Against Two Popular Mobile Networks," in Helsinki University of Technology Seminar on Network Security., 2000 .

[67] F. Sabath and H. Garbe, "Assessing the likelihood of various intentional electromagnetic environments the initial step of an iemi risk analysis," in 2015 IEEE International Symposium on Electromagnetic Compatibility (EMC), Aug 2015, pp. $1083-1088$.

[68] "NOTICE OF APPARENT LIABILITY FOR FORFEITURE ILLEGAL OPERATION OF SIGNAL JAMMING DEVICE," Federal Communications Commission (FCC), FCC 14-55, File No.: EB-FIELDSCR-13-00008645, April 2014.

[69] "NOTICE OF APPARENT LIABILITY FOR FORFEITURE," Federal Communications Commission (FCC), FCC 13-106, File No.: EB-FIELDNER-1200003665 , August 2013. 
[70] Thieves jam car locks to steal shopping, [Online, Accessed: 28 August, 2016]. Available: www.standard.co.uk/news/thieves-jam-car-locks-to-steal-shopping-6377639.html.

[71] (2016, July) Ukraine condemns Euro 2016 satellite jamming, [Online, Accessed: 28 August, 2016]. Available: http://www.broadbandtvnews.com/2016/07/08/ukraine-condemns-euro2016-satellite-jamming/.

[72] S. van de Beek and F. Leferink, "Robustness of a TETRA Base Station Receiver Against Intentional EMI," IEEE Transactions on Electromagnetic Compatibility, vol. 57, no. 3, pp. 461-469, June 2015.

[73] S. van de Beek, H. Schipper, and F. Leferink, "Intentional emi mechanisms on a wireless receiver," in European Electromagnetics International Symposium (EUROEM London, 2016), 2016.

[74] European Telecommunication Standards Institute (ETSI). [Online]. Available: http://www.etsi.org/.

[75] J. Dunlop, D. Girma, and J. Irvine, Digital Mobile Communications and the TETRA System. John Wiley \& Sons Ltd., 1999.

[76] P. Stavroulakis, Terrestrial Trunked Radio - TETRA. A Global Security Tool. Springer, 2007.

[77] Terrestrial Trunked Radio (TETRA); Voice plus Data (V+D); Part 2: Air Interface (AI), ETSI EN 300 392-2, V3.4.1, 2010.

[78] Terrestrial Trunked Radio (TETRA); Voice plus Data (V+D); Part 15: TETRA frequency bands, duplex spacings and channel numbering, ETSI TS 100 392-15, V1.4.1, 2010.

[79] S. Chennakeshu and G. Saulnier, "Differential detection of $\pi / 4$-shifted-DQPSK for digital cellular radio," Vehicular Technology, IEEE Transactions on, vol. 42, no. 1, pp. 46-57, Feb 1993.

[80] Terrestrial Trunked Radio (TETRA); Conformance testing specification; Part 1: Radio, ETSI EN 300 394-1, V3.1.1, 2007.

[81] B. Razavi, RF Microelectronics Second Edition. Pearson Education Inc., 2012.

[82] I. C. Hunter, L. Billonet, B. Jarry, and P. Guillon, "Microwave filtersapplications and technology," IEEE Transactions on Microwave Theory and Techniques, vol. 50, no. 3, pp. 794-805, Mar 2002. 
[83] T. Nilsson and R. Jonsson, "Investigation of HPM front-door protection devices and component susceptibility," FOI - Swedish Defence Research Agency, Tech. Rep. FOI-R-1771-SE, 2005.

[84] — , "Implementation of HPM Front-door Protections and Component Investigations," FOI - Swedish Defence Research Agency, Tech. Rep. FOI-R-2126-SE, 2006 .

[85] M. Höijer, M. Bäckström, R. Jonsson, T. Nilsson, O. Lundén, and Q. Wahab, "HPM - skyddsmetoder fr NBF," FOI - Swedish Defence Research Agency, Tech. Rep., 2007.

[86] A. A. Moulthrop, M. S. Muha, R. B. Dybdal, and H. J. Wintroub, "Hpm damage thresholds of gaas fets and hemts," THE AEROSPACE CORPORATION, Tech. Rep., 1992.

[87] M. Rudolph, R. Behtash, R. Doerner, K. Hirche, J. Wurfl, W. Heinrich, and G. Trankle, "Analysis of the survivability of gan low-noise amplifiers," IEEE Transactions on Microwave Theory and Techniques, vol. 55, no. 1, pp. 37-43, Jan 2007.

[88] S. Colangeli, A. Bentini, W. Ciccognani, E. Limiti, and A. Nanni, "Gan-based robust low-noise amplifiers," IEEE Transactions on Electron Devices, vol. 60, no. 10, pp. 3238-3248, Oct 2013.

[89] Terrestrial Trunked Radio (TETRA); Feasibility Study into the Implications of Operating Public Safety Sector (PSS) TEDS using the proposed "Tuning Range" concept in the $410 \mathrm{MHz}$ to $430 \mathrm{MHz}$ and $450 \mathrm{MHz}$ to $470 \mathrm{MHz}$ frequency bands, ETSI TR 102 513, V1.1.1, 2006.

[90] Y. Ming, "Power-handling capability for RF filters," Microwave Magazine, IEEE, vol. 8, no. 5, pp. 88-97, 2007.

[91] J. J. Gavan and M. B. Shulman, "Effects of densensitization on mobile radio system performance, part i: Qualitative analysis," IEEE Transactions on Vehicular Technology, vol. 33, no. 4, pp. 285-290, Nov 1984.

[92] D. C. Schleher, Electronic Warfare in the Information Age. Artech House, Inc, 1999 .

[93] W. Xu, W. Trappe, Y. Zhang, and T. Wood, "The feasibility of launching and detecting jamming attacks in wireless networks," in Proceedings of the 6th ACM International Symposium on Mobile Ad Hoc Networking and Computing, ser. MobiHoc '05. New York, NY, USA: ACM, 2005, pp. 46-57. [Online]. Available: http://doi.acm.org/10.1145/1062689.1062697 
[94] X. Wenyuan, M. Ke, W. Trappe, and Y. Zhang, "Jamming sensor networks: attack and defense strategies," Network, IEEE, vol. 20, no. 3, pp. 41-47, 2006.

[95] A. Mpitziopoulos, D. Gavalas, C. Konstantopoulos, and G. Pantziou, "A survey on jamming attacks and countermeasures in WSNs," Communications Surveys Tutorials, IEEE, vol. 11, no. 4, pp. 42-56, Fourth 2009.

[96] K. Pelechrinis, M. Iliofotou, and S. V. Krishnamurthy, "Denial of Service Attacks in Wireless Networks: The Case of Jammers," Communications Surveys E Tutorials, IEEE, vol. 13, no. 2, pp. 245-257, 2011.

[97] R. Poisel, Modern Communications Jamming Principles and Techniques, Second Edition. Norwood: Artech House, 2011.

[98] G. Breed, "Bit error rate: fundamental concepts and measurement issues," High Frequency Electronics, vol. 2, pp. 46-48, 2003.

[99] Department of Defense Interface Standard - Requirements for the Control of Electromagnetic Interference Characteristics of Subsystems and Equipment, MIL-STD-461E, 1999.

[100] G. Gong, "Wireless Security," in presented at the IEEE International Symposium on Electromagnetic Compatibility, Rayleigh, 2014.

[101] R. R. Tanuhardja, S. van de Beek, M. J. Bentum, and F. B. J. Leferink, "Vulnerability of Terrestrial-Trunked Radio to Intelligent Intentional Electromagnetic Interference," IEEE Transactions on Electromagnetic Compatibility, vol. 57, no. 3, pp. 454-460, June 2015.

[102] W. Xu, K. Ma, W. Trappe, and Y. Zhang, "Jamming sensor networks: attack and defense strategies," Network, IEEE, vol. 20, no. 3, pp. 41-47, 2006.

[103] R. P. Jover, J. Lackey, and A. Raghavan, "Enhancing the security of lte networks against jamming attacks," EURASIP Journal on Information Security, vol. 2014, no. 1, pp. 1-14, 2014. [Online]. Available: http: //dx.doi.org/10.1186/1687-417X-2014-7

[104] J. Mirkovic and P. Reiher, "A taxonomy of DDoS attack and DDoS defense mechanisms," ACM SIGCOMM Computer Communication Review, vol. 34, no. 2, pp. 39-53, 2004.

[105] J. F. Kurose and K. W. Ross, Computer Networking: A top down approach 4th edition. Upper Saddle River, NJ: Addison-Wesley Inc, 2007.

[106] (2013, Jun.) Simulink Passband Modulation. [Online]. Available: http: //www.mathworks.com/help/comm/examples/passband-modulation.html 
[107] M. Mleczko, S. Fisahn, and H. Garbe, "Measurements of EMI signals on radio links based on commercial off-the-shelf wireless devices," in 2012 International Symposium on Electromagnetic Compatibility (EMC EUROPE), Rome, 2012, pp. 1-5.

[108] S. v. d. Beek and F. Leferink, "Vulnerability of remote keyless-entry systems against pulsed electromagnetic interference and possible improvements," IEEE Transactions on Electromagnetic Compatibility, vol. PP, no. 99, pp. 1-7, 2016.

[109] S. van de Beek, R. Vogt-Ardatjew, and F. Leferink, "Robustness of remote keyless entry systems to intentional electromagnetic interference," in 2014 International Symposium on Electromagnetic Compatibility, Sept 2014, pp. 1242-1245.

[110] S. van de Beek, S. Jeunink, and F. Leferink, "Effect of pulsed interference on an ASK receiver," in 2015 IEEE International Symposium on Electromagnetic Compatibility (EMC), Aug 2015, pp. 1136-1140.

[111] Requirements of Remote Keyless Entry (RKE) Systems, Maxim Integrated, Application Note 3395, 2005.

[112] NM95HS01/NM95HS02 HiSeC High Security Rolling Code Generator Data Sheet, National Semiconductor Corporation, February 1996.

[113] A. I. Alrabady and S. M. Mahmud, "Analysis of attacks against the security of keyless-entry systems for vehicles and suggestions for improved designs," IEEE Transactions on Vehicular Technology, vol. 54, no. 1, pp. 41-50, Jan 2005.

[114] S. Kamkar, "Drive It Like You Hacked It: New Attacks and Tools to Wirelessly Steal Cars," in DEF CON 23, 2015.

[115] Anatomy of the RollJam Wireless Car Hack, [Online, Accessed: 3 May, 2016]. Available: http://makezine.com/2015/08/11/anatomy-of-the-rolljam-wireless-car-hack/.

[116] C. Melange, J. Bauwelinck, and J. Vandewege, "Low-power, super-regenerative receiver targets 433-mhz ism band," [Online]. Available: http://www.edn.com/design/analog/4321452/ Low-power-super-regenerative-receiver-targets-433-MHz-ISM-band, February 2006, Accessed on: July 9, 2016.

[117] E. Insam, "Designing super-regenerative receivers," Electronics World, April 2002 .

[118] H. Ataka, "On superregeneration of an ultra-short-wave receiver," Proceedings of the Institute of Radio Engineers, vol. 23, no. 8, pp. 841-884, Aug 1935. 
[119] TDA 5200 ASK Single Conversion Receiver Data Sheet, Infineon Technologies AG, 81726 Munich, Germany, 2010.

[120] ATA5781/ATA5781N/ATA5782/ATA5783 UHF ASK/FSK Receiver Summary Data Sheet, Atmel Corporation, 1600 Technology Drive, San Jose, CA 95110 USA, July 2015.

[121] L. E. Miller, "Tutorial: Effects of pulsed interference," IEEE P802.15 Working Group for Wireless Personal Area Networks (WPANs), Tech. Rep., 2003.

[122] S. O. Tengstrand, K. Fors, P. Stenumgaard, and K. Wiklundh, "Jamming and interference vulnerability of ieee 802.11p," in Electromagnetic Compatibility (EMC Europe), 2014 International Symposium on, 2014, pp. 533-538.

[123] T. C. Clancy, "Adaption overhead in time-varying cognitive radio channels," in Consumer Communications and Networking Conference, 2008. CCNC 2008. 5th IEEE, 2008, pp. 1009-1013.

[124] T. Rappaport, Wireless Communication: Principles and Practice. Upper Saddle River, New Jersey: Prentice Hall PTR, 1996.

[125] MAX 1470 Superheterodyne Receiver Data Sheet, Maxim Integrated Products, Sunnyvale, CA, USA, September 2014.

[126] MAX 1470 Evaluation Kit Data Sheet, Maxim Integrated Products, Sunnyvale, CA, USA, December 2002.

[127] MAX1472 Evaluation Kit Data Sheet, Maxim Integrated Products, Sunnyvale, CA, USA, November 2003.

[128] S. Haykin and M. Moher, Introduction to Analog and Digital Communications. John Wiley \& Sons, 2007.

[129] R. G. Meyer and A. K. Wong, "Blocking and desensitization in rf amplifiers," IEEE Journal of Solid-State Circuits, vol. 30, no. 8, pp. 944-946, Aug 1995.

[130] F. Hansen, "Desensitization in transistorized pm/fm-receivers," in Vehicular Technology Conference, 1967. 18th IEEE, vol. 18, Dec 1967, pp. 78-86.

[131] J. J. Gavan and M. B. Shulman, "Effects of desensitization on mobile radio system performance, part ii: Quantitative analysis," IEEE Transactions on Vehicular Technology, vol. 33, no. 4, pp. 291-300, Nov 1984.

[132] RF Bay LNA-1440, LNA Series, 10 kHz - 1400 MHz Low Noise Amplifier, RF Bay, Inc., www.rfbayinc.com, 2006. 
[133] M. McKinley, K. A. Remley, M. Mylinksi, J. Kenney, D. Schreurs, and B. Nauwelaers, "EVM Calculation for Broadband Modulated Signals," Work of United States Goverment, Tech. Rep., 2005.

[134] R. A. Shafik, M. S. Rahman, and A. R. Islam, "On the extended relationships among evm, ber and snr as performance metrics," in 2006 International Conference on Electrical and Computer Engineering, Dec 2006, pp. 408-411.

[135] J. Proakis, Digital Communications. 1221 Avnue of the Americas, New York, NY, 10020: Irwin/McGraw-HIll, 2001.

[136] A. A. Abidi, "The path to the software-defined radio receiver," IEEE Journal of Solid-State Circuits, vol. 42, no. 5, pp. 954-966, May 2007.

[137] H. Darabi, "A blocker filtering technique for saw-less wireless receivers," IEEE Journal of Solid-State Circuits, vol. 42, no. 12, pp. 2766-2773, Dec 2007.

[138] D. Murphy, H. Darabi, A. Abidi, A. A. Hafez, A. Mirzaei, M. Mikhemar, and M. C. F. Chang, "A blocker-tolerant, noise-cancelling receiver suitable for wideband wireless applications," IEEE Journal of Solid-State Circuits, vol. 47, no. 12, pp. 2943-2963, Dec 2012.

[139] A. Ghaffari, E. A. M. Klumperink, F. van Vliet, and B. Nauta, "A 4-element phased-array system with simultaneous spatial- and frequency-domain filtering at the antenna inputs," IEEE Journal of Solid-State Circuits, vol. 49, no. 6, pp. 1303-1316, June 2014.

[140] H. Darabi, D. Murphy, M. Mikhemar, and A. Mirzaei, "Blocker tolerant software defined receivers," in European Solid State Circuits Conference (ESSCIRC), ESSCIRC 2014 - 40th, Sept 2014, pp. 35-42.

[141] M. C. M. Soer, E. A. M. Klumperink, Z. Ru, F. E. van Vliet, and B. Nauta, "A 0.2-to-2.0GHz 65nm CMOS receiver without LNA achieving ¿11dBm IIP3 and ; $6.5 \mathrm{~dB}$ NF," in 2009 IEEE International Solid-State Circuits Conference - Digest of Technical Papers, Feb 2009, pp. 222-223,223a.

[142] Z. Ru, E. A. M. Klumperink, G. J. M. Wienk, and B. Nauta, "A software-defined radio receiver architecture robust to out-of-band interference," in 2009 IEEE International Solid-State Circuits Conference - Digest of Technical Papers, Feb 2009, pp. 230-231,231a.

[143] S. van de Beek, M. Stojilović, N. Mora, M. Rubinstein, F. Rachidi, and F. Leferink, "Protection Strategy against IEMI for Wireless Communication Infrastructures," in 2016 International Symposium on Electromagnetic Compatibility (EMC), 2016. 
[144] W. Xu, T. Wood, and Y. Zhang, "Channel surfing and spatial retreats: defenses against wireless denial of service," in in Proceedings of the 2004 ACM workshop on Wireless security, 2004. ACM Press, 2004, pp. 80-89.

[145] "DIEHL 2011. HPEM Case Brochure. In: DIEHL (ed.)."

[146] F. Vega, "Analytical methods for the study and design of integrated switched oscillators and antennas for mesoband radiation," Ph.D. dissertation, École Polytechnique Fédérale de Lausanne, 2013.

[147] W. Van Etten, Introduction to Random Signals and Noise. John Wiley \& Sons, Ltd, 2006. 


\section{List of Publications}

\section{Peer-reviewed journal papers}

1. S. van de Beek and F. Leferink, "Vulnerability of Remote Keyless-Entry Systems Against Pulsed Electromagnetic Interference and Possible Improvements," in IEEE Transactions on Electromagnetic Compatibility, vol. 58, no. 4, pp. 1259-1265, Aug. 2016.

This paper was an invited journal on the occasion of receiving the Best Student Paper Award at the IEEE International Symposium on EMC in Dresden, 2015.

2. R. R. Tanuhardja, S. van de Beek, M. J. Bentum and F. B. J. Leferink, "Vulnerability of Terrestrial-Trunked Radio to Intelligent Intentional Electromagnetic Interference," in IEEE Transactions on Electromagnetic Compatibility, vol. 57 , no. 3, pp. 454-460, June 2015.

3. S. van de Beek and F. Leferink, "Robustness of a TETRA Base Station Receiver Against Intentional EMI," in IEEE Transactions on Electromagnetic Compatibility, vol. 57, no. 3, pp. 461-469, June 2015.

4. S. van de Beek et al., "Overview of the European project STRUCTURES," in IEEE Electromagnetic Compatibility Magazine, vol. 3, no. 4, pp. 70-79, 4th Quarter 2014.

\section{Peer-reviewed conference papers}

1. S. van de Beek, M. Stojilović, N. Mora, M. Rubinstein, F. Rachidi and F. Leferink, "Protection Strategy against IEMI for Wireless Communication Infrastructures," in 2016 International Symposium on Electromagnetic Compatibility (EMC), Wroclaw, 2016.

This paper received the Best Paper Award. 
2. S. van de Beek, H. Schipper and F. Leferink, "Intentional EMI mechanisms on a wireless receiver," in European Electromagnetics International Symposium (EUROEM), London, 2016.

3. F. De Simio, F. De Cillis, G. Fumagalli, M. C. De Maggio, S. van de Beek, J. Dawson, et al., "Strategies to Improve Critical Infrastructures Robustness Against the IEMI Threat: A Review of Relevant Standards and Guidelines on the Topic," in Critical Information Infrastructures Security: 10th International Conference, CRITIS 2015, Berlin, Germany, October 5-7, 2015, pp. 251-254.

4. S. van de Beek, S. Jeunink and F. Leferink, "Effect of pulsed interference on an ASK receiver," 2015 IEEE International Symposium on Electromagnetic Compatibility (EMC), Dresden, 2015, pp. 1136-1140.

This paper received the Best Student Paper Award.

5. S. van de Beek et al., "The european project STRUCTURES: Challenges and results," 2015 IEEE International Symposium on Electromagnetic Compatibility (EMC), Dresden, 2015, pp. 1095-1100.

6. S. van de Beek, R. Vogt-Ardatjew and F. Leferink, "Intentional electromagnetic interference through saturation of the RF front end," 2015 Asia-Pacific Symposium on Electromagnetic Compatibility (APEMC), Taipei, 2015, pp. 132135.

7. B. Menssen, M. Mleczko, H. Garbe, K.U. Rathjen, S. Dickmann, S. van de Beek and F. Leferink, "Reference configurations for the characterization of critical infrastructures," 2014 International Symposium on Electromagnetic Compatibility, Gothenburg, 2014, pp. 1218-1223.

8. S. van de Beek, R. Vogt-Ardatjew and F. Leferink, "Robustness of remote keyless entry systems to intentional electromagnetic interference," 2014 International Symposium on Electromagnetic Compatibility, Gothenburg, 2014, pp. 1242-1245.

9. S. van de Beek and F. Leferink, "Current intentional EMI studies in Europe with a focus on STRUCTURES," 2014 International Symposium on Electromagnetic Compatibility, Tokyo (EMC'14/Tokyo), Tokyo, 2014, pp. 402-405.

10. R. Vogt-Ardatjew, S. van de Beek and F. Leferink, "Experimental extreme field strength investigation in reverberant enclosures," 2014 International Symposium on Electromagnetic Compatibility, Gothenburg, 2014, pp. 332-336.

11. R. Vogt-Ardatjew, S. van de Beek and F. Leferink, "Influence of reverberation chamber loading on extreme field strength," 2014 International Symposium on Electromagnetic Compatibility, Tokyo, 2014, pp. 685-688. 
12. S. van de Beek, K. A. Remley, C. L. Holloway, J. M. Ladbury and F. Leferink, "Characterizing large-form-factor devices in a reverberation chamber," 2013 International Symposium on Electromagnetic Compatibility (EMC EUROPE), Brugge, 2013, pp. 375-380.

13. S. van de Beek, K. A. Remley, C. L. Holloway, J. Ladbury and F. Leferink, "Effect of a metallic object on the quality factor of a reverberation chamber," 2013 Asia-Pacific Symposium on Electromagnetic Compatibility (APEMC), Melbourne, VIC, 2013, pp. 1-4.

14. R. Vogt-Ardatjew, S. van de Beek and F. Leferink, "Wide-band antennas for reverberation chambers," 2012 International Symposium on Electromagnetic Compatibility (EMC EUROPE), Rome, 2012, pp. 1-5.

15. S. van de Beek, R. Vogt-Ardatjew, H. Schipper and F. Leferink, "Vibrating Intrinsic Reverberation Chambers for shielding effectiveness measurements," 2012 International Symposium on Electromagnetic Compatibility (EMC EUROPE), Rome, 2012, pp. 1-6.

16. C. R. Dunlap, C.L. Holloway, R. Pirkl, J. Ladbury, E.F. Kuester, D.A. Hill and S. van de Beek, "Characterizing reverberation chambers by measurements of the enhanced backscatter coefficient," 2012 IEEE International Symposium on Electromagnetic Compatibility (EMC), Pittsburgh, PA, 2012, pp. 210-215. 


\section{List of Abbreviations}

$\pi / 4-D Q P S K$

$\mathrm{AACH}$

ASK

AWGN

BEP

BER

$\mathrm{BPF}$

BS

CI

COTS

DDoS

DoS

DSN

DSP

DUT

EM

EMC

EME

EMI

EMT

EVM

GPS

GSM $\pi / 4$-shifted Differential Quaternary Phase Shift Keying

Access Assignment CHannel

Amplitude-Shift Keying

Additive White Gaussian Noise

Bit-Error-Probability

Bit-Error-Ratio

Band Pass Filter

Base Station

Critical Infrastructure

Commercial-Off-The-Shelf

Distributed Denial-of-Service

Denial-of-Service

Data Slicer Negative input

Data Slicer Positive input

Device Under Test

Electromagnetic

Electromagnetic Compatibility

Electromagnetic Environment

Electromagnetic Interference

Electromagnetic Topology

Error Vector Magnitude

Global Positioning System

Global System for Mobile Communications 


\begin{tabular}{|c|c|}
\hline HPEM & High Power Electromagnetics \\
\hline HPM & High Power Microwave \\
\hline IEC & International Electrotechnical Commission \\
\hline IEMI & Intentional Electromagnetic Interference \\
\hline IF & Intermediate Frequency \\
\hline IoT & Internet of Things \\
\hline iVSG & interfering Vector Signal Generator \\
\hline JSR & Jamming-to-Signal Ratio \\
\hline LNA & Low Noise Amplifier \\
\hline LNB & Low Noise Block \\
\hline LO & Local Oscillator \\
\hline LoS & Line-of-Sight \\
\hline LTE & Long-Term Evolution \\
\hline MAC & Medium Access Control \\
\hline MS & Mobile Station \\
\hline mVSG & modulating Vector Signal Generator \\
\hline OOB & Out-Of-Band \\
\hline OOK & On-Off Keying \\
\hline OSI & Open Systems Interconnect \\
\hline PDR & Packet Delivery Ratio \\
\hline PMR & Professional Mobile Radio \\
\hline PRF & Pulse Repetition Frequency \\
\hline QAM & Quadrature Amplitude Modulation \\
\hline $\mathrm{RC}$ & Resistor-Capacitor \\
\hline $\mathrm{RF}$ & Radio Frequency \\
\hline RSSI & Received Signal Strength Indicator \\
\hline RKE & Remote Keyless-Entry \\
\hline SAW & Surface Acoustic Wave \\
\hline SCADA & Supervisory Control and Data Acquisition \\
\hline SIR & Signal-to-Interference Ratio \\
\hline SNR & Signal-to-Noise Ratio \\
\hline SRR & Super Regenerative Receiver \\
\hline TETRA & Terrestrial Trunked Radio \\
\hline
\end{tabular}


TEI

UMTS

UNI

UWB

VNA

VSA

VSG

WP
TETRA Equipment Identification

Universal Mobile Telecommunications System

User Network Interface

Ultrawideband

Vector Network Analyzer

Vector Signal Analyzer

Vector Signal Generator

Work Package 


\section{Dankwoord}

Een promotietraject is niet makkelijk en de meeste promovendi kennen momenten van crisis en wanhoop; zo ook ik. Dit zijn de momenten dat het cruciaal is dat je mensen om je heen hebt waar je steun kan vinden. Ik wil dit moment aangrijpen om enkele belangrijke personen te bedanken.

Allereerst wil ik beginnen met mijn promotor Frank Leferink. Frank, jij bent reden nummer 1 dat ik überhaupt ben begonnen met promoveren. Je hebt me de kans geboden, me overtuigd om de positie te aanvaardden en je hebt me altijd gesteund en bovenal vertrouwd. Ik ben je dankbaar voor de vrijheid die je me altijd toevertrouwde en de lange technische discussies op maandag. En natuurlijk voor de mogelijkheden die je ons bood om op conferentie te gaan. Ik heb ten volle genoten van elke "business trip". Ik hoop dat we later nog eens kunnen samenwerken. Speciale dank ook voor Frits Buesink. Jouw aanstekelijke optimisme en bijzondere kijk op de wereld helpt altijd om dingen in perspectief te zien. Daarbij ben ik je ook dankbaar voor de vele uren die je hebt gestoken in het corrigeren van me proefschrift. En Frits, sinds je optreden op mijn bruiloft zal jij voor mijn familie en vrienden altijd hét gezicht van de universiteit blijven.

Naast veel lezen en onderzoek is een wezenlijk onderdeel van promoveren ook hands-on metingen doen. Hierin heeft een jonge $\mathrm{PhD}$ kandidaat vaak hulp nodig van mensen met ervaring en het geduld om je te helpen. Ik heb veel geleerd met betrekking tot meetopstellingen binnen Thales Nederland van Hans Schipper en Ingen Mast. Hans in het bijzonder, hartelijk bedankt voor je tijd, vertrouwen en begrip. Ik ben blij dat ik veel met jou heb mogen samenwerken. Ook een woord van dank voor Eric Klumperink en Henk de Vries van ICD. Bedankt dat jullie de tijd hebben genomen om rustig naar mijn vragen en problemen te luisteren en geholpen hebben.

Vier jaar onderzoek kent altijd ups en downs, maar ik ben erg dankbaar dat ik een stabiele fijne groep om me heen had bij de Telecommunication Engineering Group. Ik heb er vele prettige collega's en studenten gekend die ik niet zal vergeten. Mark, jij bent een belangrijke reden dat ik besloot om na mijn Bachelor verder te gaan met een Master elektrotechniek bij TE. Jouw enthousiasme voor draadloze communicatie systemen is aanstekelijk en ik ben erg blij dat ik mijn bachelor opdracht voor jou 
heb mogen doen. Arjan, ook jij heb me van begin af aan betrokken bij de vakgroep en altijd de tijd genomen om te luisteren en te helpen bij ingewikkelde problemen. Immers, iedereen binnen TE weet: als het echt lastig wordt moet je bij Arjan zijn. Daarbij ben ik blij dat ik je 3 jaar heb mogen helpen met het vak Random Signalen en Ruis. Dan kom ik nu bij Lilian, de bindende factor van TE. Al die keren dat ik in je kantoor heb zitten klagen over mijn pietluttige problemen, of om gewoon gezellig te babbelen als ik geen zin had om te werken, hebben mij geholpen. Jij liet nooit merken dat je me zat werd of dat je me vond aanstellen, altijd een luisterend oor, ook al was je druk. Bedankt!

I would like to thank all my fellow (Ph.D.) students at TE. You guys (and some girls) are responsible for the great atmosphere within the group. I enjoyed the mix of so many international smart colleagues in one office. Zaher, Cees, Ben, Robert, Bart, Alex, Ibrahim, Caterina, Pieter, Niek, Dwi, Iwan, Roelof, and Olga: Thank you all! I loved the many cross-cultural discussions we had and I believe this helped me to develop as a person. I am sure we found good friends in each other. Ibrahim, thank you for teaching me how to maintain a proper beard. I profit from it every day. Alex, I appreciated you as my roomie in the last months. Sorry for all my complaints and thank you for all the lovely cookies. Niek, Robert and Roelof Grootjans, and Rowan; I enjoyed every single Friday (or Tuesday, or Monday, or any other weekday) afternoon drink with you guys. They were good and mostly necessary. Robert Vogt, you and I started our academic career simultaneously and shared office for almost 5 years. It was pleasure to work, travel and drink with you. I will miss you as a colleague.

Daarnaast is mijn leven buiten het werk en de universiteit doorslaggevend geweest om het vol te kunnen houden. Mijn vrienden, homeboys uit Voorthuizen, lieten me elke week zien waar het uiteindelijk om draait. Elke weekend was er weer een reden voor een feestje, elk weekend was weer fantastisch. Hopelijk verandert onze vriendschap niet en blijven we elkaar veel zien.

Ten slotte wil ik graag mijn familie bedanken, mijn ouders, broers en schoonzussen en mijn schoonfamilie. Jullie zijn altijd de stabiele factor in mijn leven geweest. Nuchter, geïnteresseerd en altijd daar wanneer ik jullie nodig had. Mijn ouders wil ik in het bijzonder bedanken voor de steun gedurende mijn gehele studie. Jullie weten hoe veel moeite het mij kostte om gemotiveerd te blijven; hoe vaak ik met tegenzin thuis voor school of de universiteit aan het werk ging. Juist doordat ik van jullie in mijn hele nooit druk heb ervaren om te presteren heb ik het volgehouden. Mijn grootste dank gaat uit naar mijn vrouw Gisette. Het is ongelooflijk dat je nooit tegen mij hebt geklaagd over mijn nukkige buien als het weer niet gelukt was met werk, of als ik doordeweeks weer had zitten procrastineren waardoor ik in het weekend of 's avonds moest doorwerken. Je accepteerde het allemaal, bleef altijd positief en liet mij de betekenis van onvoorwaardelijke liefde zien. Bedankt! Ik kijk met veel vertrouwen en goede zin uit naar een mooie toekomst met jou. 
Bij deze is dit boek gesloten, maar gelukkig beginnen we snel weer aan een nieuw boek. Of zoals J.R.R. Tolkien het verwoordt:

The road goes ever on and on, Down from the door where it began.

Now far ahead the Road has gone,

And I must follow if I can,

Pursuing it with eager feet,

Until it joins some larger way,

Where many paths and errands meet.

And wither then? I cannot say.

J.R.R. Tolkien 
